Review

\title{
Contact Semi-Riemannian Structures in CR Geometry: Some Aspects
}

\author{
Domenico Perrone \\ Dipartimento di Matematica e Fisica “E. De Giorgi”, Universitá del Salento, Via Provinciale Lecce-Arnesano, \\ 73100 Lecce, Italy; domenico.perrone@unisalento.it
}

Received: 26 September 2018; Accepted: 2 January 2019; Published: 9 January 2019

\begin{abstract}
There is one-to-one correspondence between contact semi-Riemannian structures $(\eta, \xi, \varphi, g)$ and non-degenerate almost $C R$ structures $(\mathcal{H}, \vartheta, J)$. In general, a non-degenerate almost $C R$ structure is not a CR structure, that is, in general the integrability condition for $\mathcal{H}^{1,0}:=\{X-i J X, X \in \mathcal{H}\}$ is not satisfied. In this paper we give a survey on some known results, with the addition of some new results, on the geometry of contact semi-Riemannian manifolds, also in the context of the geometry of Levi non-degenerate almost CR manifolds of hypersurface type, emphasizing similarities and differences with respect to the Riemannian case.
\end{abstract}

Keywords: contact semi-Riemannian structures; non-degenerate almost CR structures; tangent hyperquadric bundles; homogeneous non-degenerate CR three-manifolds; lie groups; levi-flat CR three-manifolds; bicontact metric structures; levi harmonicity

MSC: 53D15; 32V05; 22E99; 53C07; 53C30; 53C25; 53C50; 53C15; 53C43

\section{Introduction}

Contact (semi-)Riemannian geometry and (almost) CR geometry are two fields of research that have been developed independently of each other, and with different motivations. However, the two theories are quite related to each other. We note that there is not a monograph dedicated to contact semi-Riemannian structures which emphasizes its connection with the non-degenerate almost CR structures.

We can say that the contact geometry begins with Sophus Lie (1872) when he introduced the notion of a contact transformation as a geometric tool to study systems of differential equations (we refer to H. Geiges [1] for an overview of the historical origins of contact geometry).

The study of contact manifolds from the Riemannian point of view was introduced in the 60 's of the last century by the Japanese school, with S. Sasaki as leader. From then, contact manifolds equipped with Riemannian metrics have been intensively studied. The odd dimensional spheres $\mathbb{S}^{2 n+1}$ and the unit tangent sphere bundles $T_{1} M$ of Riemannian manifolds are the most known examples of contact Riemannian manifolds.

The monograph of D.E. Blair [2] and the monograph of C. Boyer and K. Galicki [3] give a wide and detailed overview of the results obtained in this framework. Contact manifolds equipped with semi-Riemannian metrics were first introduced and studied by T. Takahashi [4], who focused on the Sasakian case. In particular, the author discussed the classification of Sasakian semi-Riemannian manifolds of constant $\varphi$-sectional curvature $\kappa \neq-3$. The relevance in physics of contact semi-Riemannian structures was pointed out in K.L. Duggal [5] (see also H. Baum [6]). A systematic study of contact semi-Riemannian manifolds started with the paper of G. Calvaruso and D. Perrone [7] (see also [8]). 
The paper of S.S. Chern and J. Moser [9] on the real hypersurfaces in complex manifolds, and the works by Tanaka [10] and S. Webster [11], have made an important contribution to the development of CR geometry (also in terms of pseudohermitian geometry). Then, (almost) CR structures have drawn a great amount of interest for their connection with several different research areas in both analysis and geometry (see the monograph of S. Dragomir and G. Tomassini [12] for a wide and detailed overview of CR structures).

If $\theta$ is a contact 1 -form on an odd dimensional manifold and $J$ is an almost complex structure, i.e., $J^{2}=-I$, defined on the contact distribution $\mathcal{H}=\operatorname{ker} \theta$, such that the Levi form $L_{\theta}=d \theta(\cdot, J \cdot)$ is a non-degenerate Hermitian form $\mathcal{H}$, then $(\theta, J)$ is said to be a non-degenerate almost $C R$ structure. Different signatures of the Levi form $L_{\theta}$ correspond to different kind of geometries. There is one-to-one correspondence between contact semi-Riemannian structures and non-degenerate almost CR structures. In general, a non-degenerate almost $C R$ structure is not a $C R$ structure, that is, in general the integrability condition for $\mathcal{H}^{1,0}:=\{X-i J X, X \in \mathcal{H}\}$ is not satisfied. CR structures are considered mainly from a complex analytical point of view.

In this paper (which reflects the interests and knowledge of the author) we give a survey on some known results, with additions of some new result, on the geometry of contact semi-Riemannian manifolds, also in the context of the geometry of Levi non-degenerate almost CR manifolds of hypersurface type, emphasizing similarities and differences with respect to the Riemannian case. In particular, we explain the relationship between contact semi-Riemannian structures and non-degenerate pseudohermitian structures, describing also in some detail several important examples, like hypersurfaces of indefinite Kähler manifolds, and tangent hyperquadric bundles over semi-Riemannian manifolds.

The author believes that this paper will be useful especially to mathematician interested in contact Riemannian geometry, as developed for instance in D. Blair's book [2], who want to have a comprehensive look at the main differences between the strictly pseudo-convex setting and the semi-Riemannian setting.

\section{Contact semi-Riemannian Manifolds}

\subsection{Generality on Contact Semi-Riemannian Manifolds}

A $(2 n+1)$-dimensional manifold $M$ is said to be a contact manifold if it admits a contact form, this is, a global 1-form $\eta$ such that $\eta \wedge(d \eta)^{n} \neq 0$. Given a contact form $\eta$, there exists a unique vector field $\xi$, called the characteristic vector field or the Reeb vector field, such that $\eta(\xi)=1$ and $d \eta(\xi, \cdot)=0$. Furthermore, a semi-Riemannian metric $g$ is said to be an associated metric (for the contact form $\eta$ ) if there exists a tensor $\varphi$ of type $(1,1)$ such that

$$
\eta=\varepsilon g(\xi, \cdot), d \eta(\cdot, \cdot)=g(\cdot, \varphi \cdot), \varphi^{2}=-I+\eta \otimes \xi,
$$

and so $g(\xi, \xi)=\varepsilon= \pm 1$. In such a case, $(\eta, \xi, \varphi, g)$, or $(\eta, g)$, is called contact semi-Riemannian structure, or contact pseudo-metric structure.

An associated semi-Riemannian metric satisfies

$$
g(\varphi X, \varphi Y)=g(X, Y)-\varepsilon \eta(X) \eta(Y),
$$

and thus its signature is either $(2 p+1,2 n-2 p)$ or $(2 p, 2 n-2 p+1)$, according to whether $\xi$ is time-like or space-like respectively.

More in general, an almost contact structure on a $(2 n+1)$-dimensional manifold $M$ is a triplet $(\eta, \xi, \varphi)$, where $\varphi$ is a $(1,1)$-tensor, $\xi$ a global vector field, and $\eta$ a 1 -form, such that

$$
\varphi(\xi)=0, \quad \eta \circ \varphi=0, \quad \eta(\xi)=1, \quad \varphi^{2}=-I d+\eta \otimes \xi .
$$


To note that the properties $\varphi(\xi)=0, \eta \circ \varphi=0$, and the fact that the endomorphism $\varphi$ has rank $2 n$, are deducible from $\eta(\xi)=1$ and $\varphi^{2}=-I d+\eta \otimes \xi$ (cf. [2]). A semi-Riemannian metric $g$ on $M$ is said to be compatible with the almost contact structure $(\eta, \xi, \varphi)$ if Equation (2) is satisfied, where $\varepsilon= \pm 1$. In such a case, $(\eta, \xi, \varphi, g)$ is called almost contact semi-Riemannian structure. By Equations (2) and (3), $\eta(X)=\varepsilon g(\xi, X)$ and $g(\xi, \xi)=\varepsilon$ for any compatible metric. In particular, for (almost) contact semi-Riemannian manifolds, the characteristic vector field $\xi$ is either space-like or time-like, but can not be light-like. In the literature, (almost) contact Riemannian manifolds are also called (almost) contact metric manifolds.

In the sequel, we denote by $\nabla$ the Levi-Civita connection of a semi-Riemannian manifold $(M, g)$, by $R$ the corresponding curvature tensor, taken with the sign convention $R(X, Y)=\nabla_{[X, Y]}-\left[\nabla_{X}, \nabla_{Y}\right]$, and by Ric and $r$ the Ricci tensor and the scalar curvature respectively.

Let $(M, \eta, \xi, \varphi, g)$ be a contact semi-Riemannian $(2 n+1)$-manifold. In contact semi-Riemannian geometry, the tensor

$$
h=(1 / 2) \mathcal{L}_{\xi} \varphi,
$$

plays a fundamental role, where $\mathcal{L}$ denotes the Lie derivative. This tensor is self-adjoint, moreover we have (cf. [7])

$$
\begin{gathered}
\nabla \xi=-\varepsilon \varphi-\varphi h, \quad \nabla_{\xi} \varphi=0, \quad h \varphi=-\varphi h, \quad h \xi=0, \\
R(X, \xi) \xi=-\varphi \nabla_{\xi} h+\varphi^{2}+h^{2} \\
\operatorname{Ric}(\xi, \xi)=2 n-\operatorname{tr} h^{2} \\
\operatorname{tr} \nabla \varphi=\sum_{i=1}^{2 n+1} \varepsilon_{i}\left(\nabla_{E_{i}} \varphi\right) E_{i}=2 n \xi
\end{gathered}
$$

where $\left\{E_{1}, \ldots, E_{2 n+1}\right\}$ is an arbitrary local pseudo-orthonormal basis on $M, \varepsilon_{i}=g\left(E_{i}, E_{i}\right)$.

Change of the causal character of the Reeb vector field

There is a relationship between semi-Riemannian metrics of different signature associated to the same contact form $\eta$. Let $(\eta, \xi, \varphi, g)$ be a contact semi-Riemannian structure on $M$, with $g(\xi, \xi)=\varepsilon$. If we consider the semi-Riemannian metric $\bar{g}$ defined by

$$
\bar{g}=g-2 \varepsilon \eta \otimes \eta
$$

then Equation (1) easily implies that $(\eta, \xi, \varphi, \bar{g})$ is a contact semi-Riemannian structure on $M$. However $\bar{\varepsilon}=\bar{g}(\xi, \xi)=-\varepsilon$. Hence, the change of metric described by Equation (8) transforms an associated semi-Riemannian metric of signature $(2 p+1,2 n-2 p)$ into one of signature $(2 p, 2 n-2 p+1)$ and conversely. In particular, there exists a one-to-one correspondence between contact Riemannian manifolds and contact Lorentzian manifolds.

The Ricci tensors and the scalar curvatures of $\bar{g}$ and $g$ are related by (cf. [7])

$$
\begin{aligned}
\bar{R} i c(\xi, \xi) & =\operatorname{Ric}(\xi, \xi)=2 n-\operatorname{tr} h^{2} \\
\bar{R} i c(\xi, Y) & =\operatorname{Ric}(\xi, Y), \\
\bar{R} i c(X, Y) & =\operatorname{Ric}(X, Y)+2 \varepsilon g(\ell X, Y)+6 \varepsilon g(X, Y)+4 g(h X, Y)-2 \varepsilon g\left(h^{2} X, Y\right), \\
\bar{r} & =r-2 \varepsilon \operatorname{Ric}(\xi, \xi)+8 n \varepsilon,
\end{aligned}
$$

for all $X, Y \in \operatorname{ker} \eta$, where $\ell X:=R(X, \xi) \xi$. 
Reversing the contact semi-Riemannian structure

Let $(\eta, \xi, \varphi, g)$ be a contact semi-Riemannian structure on $M$. Then, the tensors

$$
\hat{\eta}=-\eta, \quad \hat{\zeta}=-\xi, \quad \hat{\varphi}=\varphi, \quad \hat{g}=-g
$$

define another contact semi-Riemannian structure on $M$ with $\hat{\varepsilon}=\hat{g}(\hat{\xi}, \hat{\xi})=-\varepsilon$. We shall say that the deformation of $(\eta, \xi, \varphi, g)$ defined by Equation (13) reverses the contact semi- Riemannian structure.

Special contact semi-Riemannian manifolds are the following.

- $\quad$ Sasakian (semi-Riemannian) manifolds are contact semi-Riemannian manifolds $(M, \eta, \xi, \varphi, g)$ whose almost contact structure $(\eta, \xi, \varphi)$ is normal, that is, the almost complex structure $J$ on $M \times \mathbb{R}$ defined by $J(X, f(d / d t))=(\varphi X-f \xi, \eta(X)(d / d t))$, where $f$ is a real-valued function, is integrable, i.e., the Nijenhuis tensor $[J, J]=0$, where

$$
[J, J](X, Y)=J^{2}[X, Y]+[J X, J Y]-J[J X, Y]-J[X, J Y] .
$$

The integrability condition $[J, J]=0$ is equivalent to the condition

$$
[\varphi, \varphi]+2 d \eta \otimes \xi=0 .
$$

Moreover, an almost contact semi-Riemannian manifold $(M, \eta, \xi, \varphi, g)$ is a Sasakian manifold if and only if

$$
\left(\nabla_{X} \varphi\right) Y=g(X, Y) \xi-\varepsilon \eta(Y) X .
$$

In the literature, Sasakian semi-Riemannian manifolds are also called pseudo-Sasakian manifolds. Pesudo-Sasakian manifolds can be also characterized by using cones on semi-Riemannian manifolds (see, for example, Refs. $[3,6,13,14])$. Let $(M, g)$ be a semi-Riemannian manifold. Consider $\widehat{M}=\mathbb{R}_{+} \times M$ equipped with the metric $\widehat{g}=t^{2} g+\varepsilon \mathrm{d} t \otimes \mathrm{d} t, \varepsilon= \pm 1$. Then, $(\widehat{M}, \widehat{g})$ is said to be the $\varepsilon$-cone on $M$. If $(\eta, \xi, \varphi, g)$ is a pseudo-Sasakian structure on $M$, with $g(\xi, \xi)=\varepsilon= \pm 1$, we put $\widehat{\xi}=t(\partial / \partial t)$ and define the tensor $J$ on $\widehat{M}$ by

$$
J X=\varepsilon \varphi X, \text { for } X \in \operatorname{ker} \eta, \quad J \xi=\widehat{\xi}, \quad J \widehat{\zeta}=-\xi .
$$

$J$ is an almost complex structure on $\widehat{M}$ compatible with the metric $\widehat{g}: \widehat{g}(J \cdot, J \cdot)=\widehat{g}(\cdot, \cdot)$. Moreover, $\Omega:=\varepsilon \mathrm{d}\left(t^{2} \eta\right)=+\varepsilon t^{2} \mathrm{~d} \eta$ is the Kahler 2-form of $(\widehat{M}, \widehat{g}, J): \Omega=\widehat{g}(\cdot, J \cdot)$. In fact, since $(\eta, \tilde{\zeta}, \varphi, g)$ is a contact semi-Riemannian structure, for $X, Y \in \operatorname{ker} \eta$ :

$$
\begin{aligned}
\Omega(X, Y) & =\varepsilon t^{2} \mathrm{~d} \eta(X, Y)=\varepsilon t^{2} g(X, \varphi Y)=t^{2} g(X, J Y)=\widehat{g}(X, J Y), \\
\Omega(\widehat{\xi}, \xi) & =\varepsilon 2 t \mathrm{~d} t \wedge \eta(\widehat{\xi}, \xi)=\varepsilon t \mathrm{~d} t(\widehat{\xi}) \eta(\widetilde{\xi})=\varepsilon t^{2}=\widehat{g}(\widehat{\xi}, J \xi), \\
\Omega(X, \widetilde{\zeta}) & =0=\widehat{g}(X, \widehat{\xi}), \quad \Omega(X, \widehat{\xi})=0=\widehat{g}(X, J \widehat{\xi}) .
\end{aligned}
$$

Since $\Omega$ is closed, $(\widehat{M}, J, \widehat{g})$ is an almost pseudo-Kaehler structure. By using also the Sasakian condition one can show that $J$ is parallel, that is, the structure on the cone is pseudo-Kaehler. Besides, the converse statement also holds. In other words, there is an one-to-one-correspondence beteween pseudo-Sasakian structures $(\eta, \xi, \varphi, g)$, with $g(\xi, \xi)=\varepsilon$, on $M$, and pseudo-Kaehler structures $(J, \widehat{g})$ on the $\varepsilon$-cone $\widehat{M}$. Moreover, the pseudo-Sasakian manifold is Einstein (respectively, of constant sectional curvature) if and only if the corresponding $\varepsilon$-cone $\widehat{M}$ is Ricci-flat (respectively, flat). 
- $\quad$-contact manifolds are contact semi-Riemannian manifolds $(M, \eta, \xi, \varphi, g)$ whose Reeb vector field $\xi$ is a Killing vector field, or equivalently, $h=0$. Any Sasakian semi-Riemannian manifold is $K$-contact and the converse also holds when $n=1$.

- $\quad H$-contact manifolds. The condition that $\xi$ be an eigenvector of the Ricci operator is a very natural condition in contact Riemannian geometry. Sasakian manifolds, $K$-contact manifolds, $(\kappa, \mu)$-spaces and locally $\varphi$-symmetric spaces satisfy this curvature condition. One of the more important interpretations of this condition is that of an $\mathrm{H}$-contact manifold as introduced by the present author in [15]. Recall that on a Riemannian manifold $(M, g)$, a unit vector field $V$ is said to be a harmonic vector field if $V:(M, g) \rightarrow\left(T_{1} M, \tilde{G}\right)$, where $\tilde{G}$ is the Sasaki metric (cf. Section 5.1), is a critical point for the energy functional restricted to maps defined by unit vector fields (see the recent monograph [16], and references therein). If $(M, g)$ is a semi-Riemannian manifold the same argument applies for vector fields of constant length (if is not light-like). The critical point condition which defines a harmonic vector field is: " $\bar{\Delta} V$ is collinear to $\mathrm{V}^{\prime}$, where $\bar{\Delta} V$ is the so called rough Laplacian of $V$. H-contact semi-Riemannian manifolds are contact semi-Riemannian manifolds whose Reeb vector field $\xi$ is harmonic, besides we have that (see $[15,17])$ : a contact semi-Riemannian manifold is $H$-contact if and only if $\xi$ is a Ricci eigenvector. The class of $H$-contact semi-Riemannian manifolds extends the classes of Sasakian and K-contact semi-Riemannian manifolds. Results about the classification of H-contact Riemannian three-manifolds are given in [18] and in the recent paper of Cho [19].

Remark 1. Sasakian structures, K-contact structures, and H-contact structures are preserved by the transformation Equation (8). In fact, the normality condition and the tensor $h=\frac{1}{2} \mathcal{L}_{\xi} \varphi$ do not depend on the metric, so that $(M, \eta, g)$ is Sasakian (respectively K-contact) if and only if $(M, \eta, \bar{g})$ is. Moreover, by using Equation (10), we have that $(M, \eta, g)$ is $H$-contact if and only if $(M, \eta, \bar{g})$ is $H$-contact.

A difference between the Riemannian case and the general semi-Riemannian one is the following: in both cases, from Equation (6), $\operatorname{tr} h^{2}=0$ implies $\operatorname{Ric}(\xi, \xi)=2 n$. But,

- $\quad$ K-contact Riemannian manifolds are characterized by the condition $\operatorname{Ric}(\xi, \xi)=2 n$, since it implies $\operatorname{tr} h^{2}=0$ and so, $h=0$ (because in the Riemannian case $h$ is diagonalizable);

- in the semi-Riemannian case the condition $\operatorname{tr} h^{2}=0$ does not imply $h=0$. On the other hand, there exist contact semi-Riemannian manifolds for which $\operatorname{tr} h^{2}=0$ but $h \neq 0$, and contact semi-Riemannian manifolds for which $h^{2}=0$ but $h \neq 0$ (see Examples 3 and 5).

Example 1. The hyperquadrics $\mathbb{S}_{2 s}^{2 n+1}, \mathbb{H}_{2 s-1}^{2 n+1}$ [4].

Consider the semi-Euclidean space $\mathbb{R}_{2 s}^{2 n+2}$ with the standard indefinite Kaehler structure $\left(g_{0}, J_{0}\right)$ :

$$
g_{0}(x, x)=-\sum_{i=1}^{2 s} x_{i}^{2}+\sum_{i=2 s+1}^{2 n+2} x_{i}^{2} ; J_{0} \partial_{i}=\partial_{n+1+i}, i=1, \ldots, n+1 ; J_{0} \partial_{i}=-\partial_{i-n-1}, i=n+2, \ldots, 2 n+2 .
$$

The pseudo-sphere

$$
\mathbb{S}_{2 s}^{2 n+1}(1)=\left\{x \in \mathbb{R}_{2 s}^{2 n+2}: g_{0}(x, x)=1\right\} \equiv \mathbb{R}^{2 s} \times \mathbb{S}^{2 n+1-2 s},
$$

and the pseudo-hyperbolic space

$$
\mathbb{H}_{2 s-1}^{2 n+1}(-1)=\left\{x \in \mathbb{R}_{2 s}^{2 n+2}: g_{0}(x, x)=-1\right\} \equiv \mathbb{S}^{2 s-1} \times \mathbb{R}^{2 n-2 s+2}
$$

are hyperquadrics of $\mathbb{R}_{2 s}^{2 n+2}$, both of dimension $(2 n+1)$, of index $2 s$ and $(2 s-1)$ and of constant sectional curvature 1 and -1 respectively. Such hyperquadrics have a canonical Sasakian semi-Riemannian structure $(\eta, \xi, \varphi, g)$ induced by the indefinite Kaehler structure $\left(g_{0}, J_{0}\right)$ :

$$
\xi=-J_{0} N, g=g_{0 \mid M}, \quad \eta=\varepsilon g(\xi, \cdot), \quad J_{0}=\varphi+\varepsilon g_{0}\left(J_{0}, N\right) N, \quad N_{p}=\varepsilon p,
$$


where $M=\mathbb{S}_{2 s}^{2 n+1}$ or $M=\mathbb{H}_{2 s-1}^{2 n+1}$, and the characteristic vector field $\xi$ is space-like and time-like respectively. In general, these tensors define an almost contact semi-Riemannian structure on an orintable non-degenerate hypersurface of an indefinite Kaehler manifold (see Proposition 9). Note that reversing the standard pseudo-Sasakian structure of the pseudo-hyperbolic space $\mathbb{H}_{2 s-1}^{2 n+1}(-1)$, we get a pseudo-Sasakian structure of constant sectional curvature 1, which identifies with the standard pseudo-Sasakian structure of the pseudo-sphere $\mathbb{S}_{2(n-s+1)}^{2 n+1}(1)$.

If we consider the Euclidean unit sphere $\mathbb{S}^{2 n+1}$, then $\xi_{0}=J_{0} N$ is referred as the standard Hopf vector field on $\mathbb{S}^{2 n+1}$. A tangent vector field $\xi$ on $\mathbb{S}^{2 n+1}$ is said to be a Hopf vector field if $\xi=J N$ for some orthogonal complex structure J on $\mathbb{R}^{2 n+2}$. By a result of $G$. Wiegmink, the Hopf vector fields on $\mathbb{S}^{2 n+1}$ are precisely the unit Killing vector fields (cf., for example, Section 3.1 in [16]).

\subsection{D-Homothetic Deformations and Contact Lorentzian Manifolds}

Let $(M, \eta, \xi, \varphi, g)$ be a contact semi-Riemannian manifold of dimension $2 n+1$, with $g(\xi, \xi)=\varepsilon$. Then, it is easy to check that, for any real constant $t \neq 0$, the tensors

$$
\tilde{\eta}=\eta_{t}=t \eta, \quad \tilde{\xi}=\xi_{t}=(1 / t) \xi, \quad \tilde{\varphi}=\varphi_{t}=\varphi, \quad \tilde{g}=g_{t}=t g+\varepsilon t(t-1) \eta \otimes \eta
$$

make up another contact semi-Riemannian structure on $M$, having the same contact distribution $\mathcal{D}=\operatorname{ker} \tilde{\eta}=\operatorname{ker} \eta$, called a $\mathcal{D}$-homothetic deformation (or, transverse homothety) of $(\eta, \xi, \varphi, g)$. Clearly, Equation (16) is the natural semi-Riemannian generalization of $\mathcal{D}$-homothetic deformations of a contact Riemannian structure, where one has $g(\xi, \xi)=1$ and needs to assume $t>0$ so that $\tilde{g}$ is still Riemannian [20]. Notice that $\tilde{g}(\tilde{\xi}, X)=\varepsilon \tilde{\eta}(X)$. In particular, $\tilde{\varepsilon}=\tilde{g}(\tilde{\xi}, \tilde{\xi})=g(\xi, \xi)=\varepsilon$, that is, $\mathcal{D}$-homothetic deformations preserve the causal character of the Reeb vector field. For $t<0$, if $g$ is of signature $(2 p+1,2 n-2 p)$, then $\tilde{g}$ is of signature $(2 n-2 p+1,2 p)$. The Ricci tensors and the scalar curvatures satisfy (see [7], Section 3):

$$
\begin{aligned}
& \tilde{h}=(1 / 2) \mathcal{L}_{\tilde{\zeta}} \tilde{\varphi}=(1 / t) h, \\
& \tilde{R} i c=\operatorname{Ric}-2 \varepsilon(t-1) g+2(t-1)(n t+n+1) \eta \otimes \eta+\frac{t-1}{t} g\left(\varepsilon\left(\nabla_{\xi} h\right) \varphi+2 h, \cdot\right), \\
& \tilde{r}=\frac{1}{t} r-\varepsilon \frac{t-1}{t^{2}} \operatorname{Ric}(\xi, \xi)-2 n \varepsilon \frac{(t-1)^{2}}{t^{2}} .
\end{aligned}
$$

By using Equations (14), (17) and (18), we get

Proposition 1. The classes of Sasakian, K-contact and H-contact semi-Riemannian manifolds are invariant for a D-homothetic deformation.

Recall that there is a canonical way to associate a contact Riemannian structure to a contact Lorentzian structure (and conversely). Let $\left(\eta, \xi, \varphi, g_{L}\right)$ be a contact Lorentzian structure on a smooth manifold $M$, where the Reeb vector field $\xi$ is time-like. Then,

$$
g=g_{L}+2 \eta \otimes \eta
$$

is a Riemannian metric, and is still compatible with the same contact structure $(\eta, \xi, \varphi)$. Moreover, in such case $g(\xi, \xi)=-g_{L}(\xi, \xi)=+1$. Hence, $(\eta, \xi, \varphi, g)$ is a contact Riemannian structure on $M$. We remark that

$$
g_{L}=g-2 \eta \otimes \eta=-g_{-1}
$$

where $g_{-1}$ is obtained by the $\mathcal{D}$-homothetic deformation of $g$ for $t=-1$. Consequently, the Levi-Civita connection and curvature of $g_{L}$ can be easily deduced from the formulae valid for a general $\mathcal{D}$-homothetic deformation. In particular, if $\bar{\nabla}$ is the Levi-Civita connection of $g_{L}$ and $\nabla$ is Levi-Civita connection of $g$, we have the following: 


$$
\bar{\nabla}_{X} Y=\nabla_{X} Y+2 g(h X, \varphi Y) \xi+2\{\eta(X) \varphi Y+\eta(Y) \varphi X\} .
$$

Taking into account that in the Lorentzian case the tensor $h$ is diagonalizable, for a unit vector field $X \in \operatorname{ker} \eta, h X=\lambda X$, from Equations (18) and (19) we have the following formulae

$$
\begin{aligned}
r_{L} & =r+4 n+2 \operatorname{tr} h^{2} \geq r+4 n, \\
\operatorname{Ric}_{L} & =\operatorname{Ric}+4 g-4 \eta \otimes \eta+2 g\left(\left(\nabla_{\xi} h\right) \varphi+2 h, \cdot\right) .
\end{aligned}
$$

Moreover, a contact Lorentzian manifold is Sasakian (respectively $\mathrm{K}$-contact, $\mathrm{H}$-contact) if and only if the corresponding contact Riemannian manifold is so.

A contact semi-Riemannian manifold is called $\eta$-Einstein if the Ricci tensor is given by

$$
\text { Ric }=\alpha g+\beta \eta \otimes \eta, \quad \text { where } \quad \alpha=\left(\frac{r}{2 n}+\varepsilon\left(\frac{\operatorname{tr} h^{2}}{2 n}-1\right)\right), \quad \beta=-\left(\varepsilon \frac{r}{2 n}+(2 n+1)\left(\frac{\operatorname{tr} h^{2}}{2 n}-1\right)\right) .
$$

In particular, the Ricci tensor of the $\eta$-Einstein $K$-contact Riemannian structure $(\eta, g)$ is given by

$$
\text { Ric }=\left(\frac{r}{2 n}-1\right) g+\left(-\frac{r}{2 n}+2 n+1\right) \eta \otimes \eta,
$$

where the scalar curvature $r$ is a constant when $n>1$, and $g$ is Einstein if and only if $r=2 n(2 n+1)$. Then, from Equations (20) and (21), the Ricci tensor of the corresponding Lorentzian $K$-contact structure $\left(\eta, g_{L}\right)$ is given by

$$
R i c_{L}=R i c+4 g-4 \eta \otimes \eta=\left(\frac{r_{L}}{2 n}+1\right) g_{L}+\left(\frac{r_{L}}{2 n}+2 n+1\right) \eta \otimes \eta,
$$

where the scalar curvature $r_{L}=r+4 n$ is a constant when $n>1$. Hence $\left(\eta, g_{L}\right)$ is $\eta$-Einstein $K$-contact, and $g_{L}$ is Einstein if and only if $r_{L}=-2 n(2 n+1)$.

In dimension three, every $K$-contact structure $(\eta, g)$ is automatically Sasakian and $\eta$-Einstein, and thus by Equation (22) also every K-contact Lorentzian three-manifold is automatically Sasakian and $\eta$-Einstein. Moreover, for a $K$-contact Lorentzian three-manifold, the scalar curvature $r_{L}$ and the $\varphi$-sectional curvature $H_{L}$ are related by $r_{L}=2 H_{L}-4$.

Recall that a Lorentzian Sasakian manifold $(M, g, \eta)$ is Einsteinian if and only if the cone $\widehat{M}$ is Ricci-flat. Moreover, geometries of this type are interesting because they provide examples of twistor spinors on Lorentzian manifolds (see, for example, Ref. [6,14]). In particular, in [6] a twistorial characterization of Einstein Lorentzian Sasakian manifolds is given .

If $(\eta, g)\left(\right.$ resp. $\left.\left(\eta, g_{L}\right)\right)$ is Einstein K-contact, then $\left(\eta, g_{L}\right)$ (resp. $\left.(\eta, g)\right)$ is $\eta$-Einstein K-contact. Now, we see as the $\eta$-Einstein Lorentzian $K$-contact structures are related to the Einstein Lorentzian Sasakian structures. Let $\left(\eta, g_{L}\right)$ be a Lorentzian $K$-contact structure on $M$ with $\xi$ time-like, $\operatorname{dim} M=2 n+1>3$. For the new Lorentzian $K$-contact structure

$$
\tilde{\eta}=t \eta, \quad \tilde{\xi}=\frac{1}{t} \xi, \quad \tilde{\varphi}=\varphi, \quad \tilde{g}_{L}=\left(g_{L}\right)_{t}=t g_{L}-t(t-1) \eta \otimes \eta=\left(g_{t}\right)_{L}, t>0,
$$

from Equations (18) and (19) we have

$$
\tilde{R} i c_{L}=R i c_{L}+2(t-1) g_{L}+2(t-1)(n t+n+1) \eta \otimes \eta, \quad \tilde{r}_{L}=\frac{r_{L}-2 n}{t}+2 n .
$$

If in addition $\left(\eta, g_{L}\right)$ is $\eta$-Einstein, since $n>1$, then the scalar curvature $r_{L}$ is a constant and the Ricci tensor of the new Lorentzian $K$-contact structure $\left(\tilde{\eta}, \tilde{g}_{L}\right)$ is given by 


$$
\begin{aligned}
\tilde{R} i c_{L} & =\left(\frac{r_{L}}{2 n}+2 t-1\right) g_{L}+\left(\frac{r_{L}}{2 n}+2 n+1+2(t-1)(n t+n+1)\right) \eta \otimes \eta, \\
& =\left(\frac{\tilde{r}_{L}}{2 n}+1\right) \tilde{g}_{L}+\left(\frac{\tilde{r}_{L}}{2 n}+2 n+1\right) \tilde{\eta} \otimes \tilde{\eta} .
\end{aligned}
$$

So, for any $t>0$, the Lorentzian $K$-contact structure $\left(\tilde{\eta}, \tilde{g}_{L}\right)$ is $\tilde{\eta}$-Einstein. Recall that the function $\hat{r}=r_{L}-2 n=r+2 n$ is the so-called Webster scalar curvature of $(\eta, g)$ and $\left(\eta, g_{L}\right)$ (see Section 3.3). Now, if the scalar curvature $r_{L}$ of the $\eta$-Einstein Lorentzian $K$-contact manifold $\left(\eta, g_{L}\right)$ satisfies $r_{L}<2 n$, i.e., $\hat{r}<0$, then the Lorentzian $K$-contact structure $\left(\tilde{\eta}=\eta_{t}, \tilde{g}_{L}=\left(g_{L}\right)_{t}\right)$ obtained in correspondence to

$$
t=-\frac{\hat{r}}{4 n(n+1)}>0
$$

is Einstein. Analogously, if $(\eta, g)$ is $\eta$-Einstein $K$-contact and $\hat{r}>0$, i.e., $r>-2 n$, equivalently $r_{L}>2 n$, the Riemannian $K$-contact structure $\left(\eta_{t}, g_{t}\right)$ obtained in correspondence to

$$
t=\frac{\hat{r}}{4 n(n+1)}>0
$$

is Einstein, and hence, when $M$ is compact, $g_{t}$ is Sasakian-Einsten (cf. [21], Theorem $\mathrm{A}^{\prime}$ ). If $r_{L}=2 n$, that is $r=-2 n$, and $M$ is compact, from Theorem 7.2 of [21], we get that the contact Riemannian structure $(\eta, g)$ which corresponds to the $\eta$-Einstein Lorentzian $K$-contact structure $\left(g_{L}, \eta\right)$ is $\eta$-Einstein Sasakian. So, summing up we get (see also [22], Section 5)

Theorem 1. Let $\left(M, \eta, \xi, \varphi, g_{L}\right)$ be an $\eta$-Einstein K-contact Lorentzian manifold of dimension $2 n+1>3$. Then, the Webster scalar curvature $\hat{r}$ is a constant and we have the following.

- If the Webster scalar curvature $\hat{r}<0$, i.e., $r_{L}<2 n$, then there exists a transverse homothety whose resulting structure $\left(\tilde{\eta}, \tilde{\xi}, \tilde{\varphi}, \tilde{g}_{L}\right)$ is Einstein-Lorentzian K-contact.

- If the Webster scalar curvature $\hat{r}>0$, i.e., $r_{L}>2 n$, then there exists a transverse homothety whose resulting structure $\left(\eta_{t}, \xi_{t}, \varphi, g_{t}\right)$ is Einstein Riemannian K-contact. If in addition $M$ is compact, $\left(\eta_{t}, g_{t}\right)$ is Sasakian-Einsten and $\left(\eta_{t},\left(g_{t}\right)_{L}\right)$ is $\eta$-Einstein Lorentzian-Sasakian.

- If the Webster scalar curvature $\hat{r}=0$, i.e., $r_{L}=2 n$, and $M$ is compact, then the structure $\left(\eta, g_{L}\right)$ is $\eta$-Einstein Lorentzian-Sasakian.

From this Theorem and Proposition 6.2 of [6], we get the following

Theorem 2. ([22]) Let $\left(M, \eta, \xi, g_{L}\right)$ be a simply connected $\eta$-Einstein Lorentzian Sasakian manifold of dimension $2 n+1>3$ and with scalar curvature $r_{L}<2 n$, i.e., $\hat{r}<0$. Then, there exists a transverse homothety whose resulting Lorentzian manifold $\left(M, \tilde{g}_{L}\right)$ is a spin manifold. Moreover, there exists a twistor spinor $\phi$ which is an imaginary Killing spinor and the associated vector field $V_{\phi}$ (the Dirac current) is $\tilde{\xi}$.

Any connected sum of $\mathbb{S}^{2} \times \mathbb{S}^{3}$ admits a Lorentzian Sasaki-Einstein structure [23]. Now, we give the following.

Example 2. Let $\Omega$ be a simply connected bounded domain in $\mathbb{C}^{n}$, equipped with the Kaehler structure $(G, J)$ of constant holomorphic sectional curvature $\kappa<-3$. The corresponding the Kaehler form $\omega$ is closed and thus $\omega=\mathrm{d} \vartheta$. Let $\pi: M=\Omega \times \mathbb{R} \rightarrow \Omega$ be the natural projection, and the coordinate on $\mathbb{R}$. We construct a Lorentzian-Sasakian structure on $M$ like the Riemannian case (cf. [2], Ch.7)). We define $\eta=\pi^{*} \vartheta+\mathrm{d} t, \quad \xi=\partial / \partial t, \quad g_{L}=\pi^{*} G-\eta \otimes \eta$, and define $\varphi$ such that to be the horizontal lift of the complex structure $J$ and zero in the vertical direction. Then, $\left(\eta, \xi, \varphi, g_{L}\right)$ is an $\eta$-Einstein Lorentzian-Sasakian structure with $\xi$ time-like. Moreover, the scalar curvature is given by $r_{L}=(n(2 n+1)(\kappa+3)+n(\kappa+7)) / 2$, and hence $r_{L}-2 n=n(n+1)(\kappa+3)<0$. Then, for $t=-\frac{\kappa+3}{4}>0$ the resulting structure $\left(\tilde{\eta}, \tilde{g}_{L}\right)$ is Einstein-Lorentzian Sasakian. 


\subsection{Curvature of K-Contact (and Sasakian) Semi-Riemannian Manifolds}

In the contact Riemannian case, the following curvature condition

$$
R(X, Y) \xi=\eta(X) Y-\eta(Y) X
$$

characterizes the Sasakian structures. In the semi-Riemannian case any Sasakian manifold satisfies Equation (23), but there is not a proof for the conversely and we do not know examples of non-Sasakian contact semi-Riemannian manifolds which satisfy Equation (23). A partial result for this problem is given by the following (cf. [22]).

Theorem 3. Let $(M, \eta, \xi, \varphi, g)$ be a K-contact semi-Riemannian manifold. Then, $M$ is Sasakian if and only if the curvature tensor $R$ satisfies Equation (23).

Moreover, for a $K$-contact semi-Riemannian manifold, $\xi$ is an eigenvector of the Ricci operator $Q: Q \xi=2 n \varepsilon \xi$. In the Riemannian case, this condition holds in a stronger form: $\mathrm{M}$ is $\mathrm{K}$-contact if and only if $Q \xi=2 n \xi$ (cf. [2], Theorem 7.1 and Proposition 7.2). Always for a K-contact semi-Riemannian manifold, by (5), one gets $R(\cdot, \xi) \xi=\varphi^{2}$. Then, for a non-degenerate plane $\operatorname{section} \operatorname{span}\left(\xi_{p}, X_{p}\right)$, $g\left(\xi_{p}, X_{p}\right)=0$, the sectional curvature

$$
K\left(\xi_{p}, X_{p}\right)=-\frac{g\left(R\left(X_{p}, \xi_{p}\right) \xi_{p}, X_{p}\right)}{\varepsilon g\left(X_{p}, X_{p}\right)}=-\frac{g\left(\varphi^{2} X_{p}, X_{p}\right)}{\varepsilon g\left(X_{p}, X_{p}\right)}=\varepsilon
$$

Conversely, we have the following (cf. [22]).

Theorem 4. If a semi-Riemannian manifold $(M, g)$ admits a Killing vector field $\xi, g(\xi, \xi)=\varepsilon= \pm 1$, such that the sectional curvature of all nondegenerate plane sections containing $\xi$ equals $\varepsilon$, then

$$
(\eta=\varepsilon g(\xi, \cdot), \xi, \varphi=-\varepsilon \nabla \xi, g)
$$

is K-contact semi-Riemannian structure on $M$.

In the same paper [22], we proved

Theorem 5. Any conformally flat K-contact semi-Riemannian manifold is Sasakian and of constant sectional curvature $\kappa=\varepsilon$.

If $(M, \eta, g)$ is a locally symmetric contact semi-Riemannian three-manifold, then $(M, g)$ is either flat or of constant sectional curvature $\kappa=\varepsilon=g(\xi, \xi)$ [7]. In particular, the pseudoEuclidean space $\mathbb{R}_{1}^{3}$ and the universal covering of the pseudohyperbolic space $\mathbb{H}_{1}^{3}(-1)$, are the only three-dimensional symply connected symmetric contact Lorentzian manifolds. Note that the pseudo-sphere $\mathbb{S}_{2}^{3}(1)$, which admits a symmetric contact semi-Riemannian structure $(\eta, g)$, with $\varepsilon=+1$ and $g$ of signature $(-,-,+)$, is nothing but the pseudohyperbolic space $\mathbb{H}_{1}^{3}(-1)$ with the reversed structure.

Now, we give the following new result

Theorem 6. Any locally symmetric K-contact semi-Riemannian manifold $(M, \eta, \xi, \varphi, g)$ is Sasakian and of constant sectional curvature $\varepsilon$.

Proof. For a K-contact semi-Riemannian manifold, from Equations (4) and (5), we have

$$
R(Y, \xi) \xi=-Y+\eta(Y) \xi \text { and } \nabla \xi=-\varepsilon \varphi \text {. }
$$


Moreover, for a locally symmetric semi-Riemannian manifold $\nabla R=0$. Then we get

$$
\begin{aligned}
0 & \left.=\left(\nabla_{X} R\right)(Y, \xi, \xi)=\nabla_{X} R(Y, \xi) \xi-R\left(\nabla_{X} Y, \xi\right) \xi-R\left(Y, \nabla_{X} \xi\right) \xi\right)-R(Y, \xi) \nabla_{X} \xi \\
& =\ldots \\
& =-\varepsilon^{2} g(\varphi X, Y) \xi-\varepsilon \eta(Y) \varphi X+\varepsilon R(Y, \varphi X) \xi+\varepsilon R(Y, \xi) \varphi X
\end{aligned}
$$

that is,

$$
R(Y,-\varphi X) \xi-R(Y, \xi) \varphi X=-\varepsilon g(\varphi X, Y) \xi-\eta(Y) \varphi X .
$$

Replacing $X$ by $\varphi X$, we have

$$
R(Y, X) \xi+R(Y, \xi) X-2 \eta(X) R(Y, \xi) \xi=\eta(Y) X-2 \eta(X) \eta(Y) \xi+\varepsilon g(X, Y) \xi
$$

that is,

$$
R(Y, X) \xi+R(Y, \xi) X=-2 \eta(X) Y+\eta(Y) X+\varepsilon g(X, Y) \xi
$$

equivalently,

$$
R(X, Y) \xi=R(\xi, X) Y-2 \eta(Y) X+\eta(X) Y+\varepsilon g(X, Y) \xi .
$$

By using this last equation, we get

$$
\begin{aligned}
0 & =\left(\nabla_{Z} R\right)(X, Y, \xi)=\nabla_{Z} R(X, Y) \xi-R\left(\nabla_{Z} X, Y\right) \xi-R\left(X, \nabla_{Z} Y\right) \xi-R(X, Y) \nabla_{Z} \xi \\
& =\ldots \\
& =-\varepsilon R(\varphi Z, X) Y+2 g(\varphi Z, Y) X-g(\varphi Z, X) Y-g(X, Y) \varphi Z+\varepsilon R(X, Y) \varphi Z,
\end{aligned}
$$

that is,

$$
-\varepsilon R(\varphi Z, X) Y+\varepsilon R(X, Y) \varphi Z=-2 g(\varphi Z, Y) X+g(\varphi Z, X) Y+g(X, Y) \varphi Z .
$$

Replacing $Z$ by $\varphi Z$, the above equation becomes

$$
\begin{gathered}
\varepsilon(R(Z, X) Y-R(X, Y) Z)+\varepsilon \eta(Z)(R(X, Y) \xi-R(\xi, X) Y)= \\
-g(X, Y) Z-g(X, Z) Y+2 g(Y, Z) X+\varepsilon \eta(Z)(-2 \eta(Y) X+\eta(X) Y+\varepsilon g(X, Y) \xi) .
\end{gathered}
$$

Then, by using Equation (24), we obtain

$$
\varepsilon(R(Z, X) Y-R(X, Y) Z)=-g(X, Y) Z-g(X, Z) Y+2 g(Y, Z) X
$$

and hence

$$
\varepsilon R(X, Y, Z, Y)=g(X, Y) g(Z, Y)+g(X, Z) g(Y, Y)-2 g(Y, Z) g(X, Y) .
$$

Now, let $p$ be an arbitrary point and $\operatorname{span}\left(X_{p}, Y_{p}\right)$ be an arbitrary non-degenerate plane. Then, from Equation (25), we obtain

$$
\varepsilon R(X, Y, X, Y)=g(X, X) g(Y, Y)-g(X, Y)^{2},
$$

that is,

$$
\frac{R\left(X_{p}, Y_{p}, X_{p}, Y_{p}\right)}{g\left(X_{p}, X_{p}\right) g\left(Y_{p}, Y_{p}\right)-g\left(X_{p}, Y_{p}\right)^{2}}=\varepsilon .
$$

Therefore $(M, \eta, \xi, \varphi, g)$ is a $K$-contact semi-Riemannian manifold of constant curvature $\varepsilon$. Then, by using Theorem 5 , we conclude that the manifolds is also Sasakian.

Remark 2. Theorems 4-6, which include in particular the Lorentzian case, give results analogous to the Riemannian case (see [2]). 
If $(M, \tilde{g})$ is a semi-Riemannian manifold which admits a Killing vector field $X_{0}$ of constant length, $g\left(X_{0}, X_{0}\right)=c \neq 0$, such that the sectional curvature of all non-degenerate plane sections containing $X_{0}$ equals $c$, then $\xi=(1 / \varepsilon c) X_{0}$ and $g=\varepsilon c \tilde{g}$, where $\varepsilon=+1$ if $c>0$ and $\varepsilon=-1$ if $c<0$, satisfy the conditions of Theorem 4 . Then, by using Theorems $4-6$, we get the following (which extends Corollary 4.3 of [22]).

Theorem 7. Let $(M, g)$ be a semi-Riemannian manifold whose admits a Killing vector field $X_{0}$ of constant length, $g\left(X_{0}, X_{0}\right)=c \neq 0$, such that the sectional curvature of all non-degenerate plane sections containing $X_{0}$ equals $c$. Then, the following properties are equivalent

Numbered lists can be added as follows:

(1) $(M, g)$ is conformally flat ;

(2) $\quad(M, g)$ is locally symmetric;

(3) $(M, g)$ is of constant sectional curvature $\kappa=c$.

\subsection{Geometry of H-Contact Semi-Riemannian Manifolds}

$H$-contact semi-Riemannian manifolds are related to the contact semi-Riemannian manifolds whose Reeb vector field is an infinitesimal harmonic transformation. Recall that a vector field $V$ on a semi-Riemannian manifold $(M, g)$ is called an infinitesimal harmonic transformation (in short i.h.t.) if the one-parameter group of local transformations generated by $V$ are local harmonic diffeomorphisms. Moreover, $V$ is an i.h.t. if and only if $\operatorname{tr}\left(L_{V} \nabla\right)=0$ (see [24,25]), where

$$
\left(L_{V} \nabla\right)(X, Y)=R(V, X) Y+\nabla_{X} \nabla_{Y} V-\nabla_{\nabla_{X} Y} V
$$

for all tangent vector fields $X, Y$. With respect to a pseudo-orthonormal basis $\left\{E_{1}, \ldots, E_{m}\right\}$ of $(M, g)$, we have

$$
\begin{aligned}
\operatorname{tr}\left(L_{V} \nabla\right) & =\sum_{i} \varepsilon_{i}\left(L_{V} \nabla\right)\left(E_{i}, E_{i}\right) \\
& =\sum_{i} \varepsilon_{i} R\left(V, E_{i}\right) E_{i}+\sum_{i} \varepsilon_{i}\left(\nabla_{E_{i}} \nabla_{E_{i}} V-\nabla_{\nabla_{E_{i}} E_{i}} V\right) \\
& =-Q V+\bar{\Delta} V,
\end{aligned}
$$

where $\bar{\Delta}$ is the rough Laplacian. Thus, a vector field $V$ is an i.h.t. if and only if $\bar{\Delta} V=Q V$.

Now, let $(M, \eta, g, \xi, \varphi)$ be an arbitrary contact semi-Riemannian manifold. Then, we have (cf. $[15,17])$

$$
\bar{\Delta} \xi=4 n \varepsilon \xi-Q \xi=\varepsilon\|\nabla \xi\|^{2} \xi-\operatorname{pr}_{\mid \operatorname{ker} \eta} Q \xi .
$$

Besides, by using Equation (6), we get

$\xi$ is an i.h.t. $\Longleftrightarrow \bar{\Delta} \xi=Q \xi \quad \Longleftrightarrow \quad Q \xi=2 n \varepsilon \xi \quad \Longleftrightarrow \quad \operatorname{tr} h^{2}=0 \quad$ and $\quad Q \xi$ is collinear to $\xi$,

from which we get the following (cf. [17]).

Theorem 8. Let $(M, \eta, g, \xi, \varphi)$ be a contact semi-Riemannian manifold. Then, the following properties are equivalent:

(1) $Q \xi=2 n \varepsilon \xi$;

(2) $\xi$ is an infinitesimal harmonic transformation;

(3) $\quad M$ is H-contact and $\operatorname{tr} h^{2}=0$.

In the Riemannian case, $t r h^{2}=0$ is equivalent to $h=0$, and so ([26]).

$\xi$ is an i.h.t. if and only if $\mathrm{M}$ is $\mathrm{K}$-contact.

In the semi-Riemannian case:

§ i.h.t. does not imply K-contact. 
In fact, the following is an example of contact semi-Riemannian manifold where $\xi$ is an i.h.t. but it is not Killing.

Example 3. ([17]) We consider the 5-dimensional connected Lie group $G$, whose Lie algebra $\mathfrak{g}$ admits a basis $\left\{E_{0}, E_{1}, E_{2}, V_{1}, V_{2}\right\}$ such that

$$
\begin{aligned}
& {\left[E_{0}, E_{1}\right]=\left[E_{0}, E_{2}\right]=-E_{2}, \quad\left[E_{0}, V_{1}\right]=-\left[E_{0}, V_{2}\right]=v_{1}, \quad\left[E_{1}, V_{1}\right]=2 E_{0}, \quad\left[E_{2}, V_{2}\right]=-2 E_{0},} \\
& {\left[E_{i}, E_{j}\right]=\left[V_{i}, V_{j}\right]=\left[E_{i}, V_{j}\right]=0, i \neq j=1,2 .}
\end{aligned}
$$

Consider the semi-Riemannian left-invariant metric $g$, for which $\left\{E_{0}, E_{i}, V_{i}\right\}$ is a pseudo-orthonormal basis with

$$
g\left(E_{0}, E_{0}\right)=\varepsilon, \quad g\left(E_{1}, E_{1}\right)=g\left(V_{1}, V_{1}\right)=1, \quad g\left(E_{2}, E_{2}\right)=g\left(V_{2}, V_{2}\right)=-1
$$

Define the left-invariant tensors $\xi, \eta$, and $\varphi$ on $G$ putting

$$
\xi=E_{0}, \quad \eta(X)=\varepsilon g(\xi, X), \quad \varphi E_{i}=V_{i}, \quad \varphi V_{i}=-E_{i}, \quad i=1,2 .
$$

Then, the metric $g$ described in Equation (28), together with tensors described in Equation (29), define a left-invariant contact semi-Riemannian structure $(\eta, g, \xi, \varphi)$ on $G$. This contact semi-Riemannian structure is $H$-contact and satisfies $t r h^{2}=0$ with $h \neq 0$ (more precisely, $2 h^{2} E_{1}=E_{2}, 2 h^{2} E_{2}=-E_{1}, 2 h^{2} V_{1}=V_{2}$, $\left.2 h^{2} V_{2}=-V_{1}\right)$. Thus, $\xi$ is an i.h.t. and $(\eta, \xi, \varphi, g)$ is not K-contact.

Remark 3. The class of contact semi-Riemannian manifolds with $\xi$ i.h.t. is invariant for $\mathcal{D}$-deformations. In fact, the class of H-contact semi-Riemannian manifolds is invariant and $t r h_{t}^{2}=\left(1 / t^{2}\right) \operatorname{trh}^{2}$.

The Lorentzian case. Let $(M, \eta, \xi, \varphi, g)$ be a contact semi-Riemannian manifold, and $\bar{g}$ the metric associated to $(\eta, \xi, \varphi)$ described by Equation (8). Then, as remarked in Section 2, $(M, \eta, \xi, \varphi, \bar{g})$ is $H$-contact if and only if $(M, \eta, \xi, \varphi, g)$ is $H$-contact. In particular, there exists a one-to-one correspondence between $H$-contact Riemannian manifolds and $H$-contact Lorentzian manifolds. It follows that the class of $H$-contact Lorentzian manifolds is really large. To note that just like in the Riemannian case, for a contact Lorentzian manifold, with $\xi$ time-like one has $\operatorname{tr} h^{2}=0$ if and only if $h=0$ [7]. Hence, using Equation (8) and the corresponding result valid in the Riemannian case ([26], [Theorem 4.1]), we have the following result.

Proposition 2. Let $(M, \eta, \xi, \varphi, g)$ be a contact Lorentzian manifold with $\xi$ time-like. Then, the following properties are equivalent:
(1) $M$ is K-contact;
(2) $\xi$ is an infinitesimal harmonic transformation;
(3) $\bar{\Delta} \xi=-2 n \xi$.

Remark 4. Let $(M, g)$ be a Lorentzian manifold and $V$ a unit time-like vector field on $M$. The space-like energy of $V$ is defined as the integral of the square norm of the restriction of $\nabla V$ to the space-like distribution $V^{\perp}$. A unit time-like vector field $V$, which is a critical point of the space-like energy, is called a spatially harmonic vector field. If $V$ is a time-like unit geodesic vector field, then it is spatially harmonic if and only if it is a harmonic vector field ([16], Chapter 8 and [27]). On the other hand, the Reeb vector field of a contact semi-Riemannian manifold is geodesic. Thus, we have the following result [17]: A contact Lorentzian manifold, with $\xi$ time-like, is $H$-contact if and only if $\xi$ spatially harmonic.

Remark 5. We note that the Reeb vector field of a three-dimensional contact Riemannian manifold $\left(M^{3}, \eta, \xi, \varphi, g\right)$ defines a harmonic map from $M$ to $T_{1} M$ if and only if it is $H$-contact and $\xi(\lambda)=0$, where $\lambda,-\lambda$ are the nontrivial eigenvalues of tensor $h$ [18]. The same characterization holds in the contact Lorentzian case (in fact, for the corresponding contact Lorentzian manifold we have $\bar{h}=h$ ). Then, it is natural to ask which are the $H$-contact Lorentzian three-manifolds for which $\lambda$ is a constant (equivalently, the Ricci eigenvalue related to 
$\xi$ is constant). In the Riemannian case, it follows from the proof of Theorem 1.2 in [18] that a three-dimensional contact Riemannian manifold is H-contact with constant Ricci eigenvalue if and only if either it is Sasakian or is locally isometric to a unimodular Lie group $G$ equipped with a non-Sasakian left-invariant contact metric structure. Then, a contact Lorentzian three-manifold is $H$-contact with constant Ricci eigenvalue (related to $\xi$ ) if and only if either it is Sasakian or is locally isometric to a unimodular Lie group $G$ equipped with a non-Sasakian left-invariant contact Lorentzian structure. A complete classification of simply connected homogeneous contact Lorentzian three-manifolds will be given in Section 4.

Since the work of Hamilton and especially Perelman's proof of the Poincaré conjecture, there has been considerable interest in the Ricci flow and its applications. For an introduction to Ricci flow we refer to the book of B. Chow and D. Knopf [28]. Ricci solitons have been intensively studied in recent years, particularly because of their relationship with the Ricci flow. For examples and more details on Ricci solitons in semi-Riemannian settings, we may refer for example to [29] and references therein. A Ricci soliton is a semi-Riemannian manifold $(M, g)$, admitting a vector field $V$, such that

$$
\text { Ric }+\frac{1}{2} \mathcal{L}_{V} g=\mu g,
$$

for some real constant $\mu$. A Ricci soliton is said to be shrinking, steady, or expanding, according to whether $\mu>0, \mu=0$ or $\mu<0$, respectively. Clearly, an Einstein manifold, together with a Killing vector field, is a trivial solution of Equation (30). As proved in the paper [30], any Riemannian Ricci soliton is an infinitesimal harmonic transformation, and it is easily seen that the same argument applies to the semi-Riemannian case. By definition, a contact (semi-Riemannian) Ricci soliton is a contact semi-Riemannian manifold $(M, \eta, \xi, \varphi, g)$, for which Equation (30) is satisfied by $V=\xi$. Since $\left(\mathcal{L}_{\xi} g\right)(\xi, X)=0$, from Equation (30) with $V=\xi$, we have that the Reeb vector field of a contact semi-Riemannian manifold satisfies

$$
Q \xi=\mu \xi
$$

So, a contact semi-Riemannian Ricci soliton is H-contact with constant Ricci eigenvalue. On the other hand, if $(M, \eta, \xi, \varphi, g)$ is a contact semi-Riemannian Ricci soliton, then $\xi$ is an infinitesimal harmonic transformation. Hence, by Theorem $8, Q \xi=\mu \xi$ with $\mu=2 n \varepsilon= \pm 2 n$ and we get the following result [17]:

Theorem 9. $A(2 n+1)$-dimensional contact semi-Riemannian Ricci soliton is $H$-contact: $Q \xi= \pm 2 n \xi$, and it is either shrinking or expanding, according to the causal character of the Reeb vector field.

In Riemannian setting, the above Theorem yields a much stronger rigidity result. In fact, by Theorem 8 we have $\operatorname{tr} h^{2}=0$, that is $h=0$. So, by using Equation (30), we have the following result (see [31] and also [22]).

Corollary 1. A contact Riemannian manifold is a contact Ricci soliton if and only if it is K-contact and Einstein.

Recall the following result of C. Boyer and K. Galicki (see [21]): A compact K-contact Einstein manifold is Sasakian Einstein. Therefore, from Corollary 1 we get the following

Theorem 10. A compact, contact Riemannian Ricci soliton is Sasakian Einstein.

Moreover, by Theorem 9 and Proposition 2, we deduce the following Lorentzian analogue of Corollary 1.

Corollary 2. Let $(M, \eta, \xi, g, \varphi)$ be a contact Lorentzian manifold with $\xi$ time-like. Then, $(M, \eta, \xi, \varphi, g)$ is a contact Ricci soliton if and only if it is Einstein and K-contact. 
By Corollary 1, only trivial contact Ricci solitons occur in Riemannian settings. On the other hand, the above Theorem 9 specifies that semi-Riemannian Ricci solitons must be found among $H$-contact manifolds, but this does not exclude the existence of nontrivial contact semi-Riemannian Ricci solitons. As explicitly remarked in [17], the left-invariant contact semi-Riemannian structure described in Example 3 is $\mathrm{H}$-contact (and $\xi$ is also an infinitesimal harmonic transformation), but not a contact Ricci soliton. Hence, the class of semi-Riemannian contact Ricci solitons is strictly included in the one of $H$-contact semi-Riemannian manifolds satisfying $Q \xi= \pm 2 n \xi$.

\section{Non-Degenerate Almost CR Structures}

Almost CR structures have drawn a great amount of interest for their connection with several different research areas in both analysis and geometry (Dragomir-Tomassini [12]). In this Section we will emphasize some aspects of their connection with the contact semi-Riemannia structures.

\subsection{Generality on Almost CR Structures}

Let $M$ be a $(2 n+1)$-dimensional manifold. An almost $C R$ structure (of hypersurface type) on $M$ is a pair $(\mathcal{H}=H(M), J)$ where $\mathcal{H}$ is a smooth real subbundle of rank $2 n$ of the tangent bundle $T M$ (also called the Levi distribution), and $J: \mathcal{H} \rightarrow \mathcal{H}$ is an almost complex structure: $J^{2}=-I$.

Example 4. Any odd-dimensional Lie group $G$ admits a left-invariant almost $C R$ structure. If $\left(\xi, X_{i}, Y_{i}\right)$, $i=1, \ldots, n$, is a basis of the Lie algebra $\mathfrak{g}$ of $G$ and define $\mathcal{H}=\operatorname{span}\left(X_{i}, Y_{i}\right)$ and $J$ by $J X_{i}=Y_{i}, J Y_{i}=-X_{i}$, then $(\mathcal{H}, J)$ is a left invariant almost $C R$ structure on $G$.

Starting from an almost contact structure $(\eta, \xi, \varphi)$, the pair $\left(\mathcal{H}=\operatorname{ker} \eta, J=\varphi_{\mid \mathcal{H}}\right)$ defines a corresponding almost $\mathrm{CR}$ structure on $M$. It is a natural question to ask when an almost CR structure $(\mathcal{H}, J)$ permits to reconstruct an almost contact structure $(\eta, \xi, \varphi)$, such that $\left(\mathcal{H}=\operatorname{ker} \eta, J=\varphi_{\mid \mathcal{H}}\right)$. The answer is given by the following result.

Proposition 3. Let $M$ denote an odd-dimensional manifold. An almost $C R$ structure $(\mathcal{H}, J)$ on $M$ is induced by an almost contact structure $(\eta, \xi, \varphi)$ if and only if $M$ admits a (globally defined) vector field $X_{0}$, transversal to $\mathcal{H}$ at any point.

Proof. If $\left(\mathcal{H}=\operatorname{ker} \eta, J=\varphi_{\mid \mathcal{H}}\right)$ for some almost contact structure $(\eta, \xi, \varphi)$, then clearly the characteristic vector field $\xi$ satisfies the requirements above.

Conversely, suppose that $(\mathcal{H}, J)$ is an almost CR structure, admitting a global vector field $X_{0}$ transversal to it. Then, it is enough to define $\xi, \eta$ and $\varphi$ by

$$
\xi=X_{0}, \quad \eta(\xi)=1, \quad \eta(X)=0, \quad \varphi(\xi)=0, \quad \varphi(X)=J(X),
$$

for any vector field $X \in \mathcal{H}$. It is then easy to check that $(\eta, \xi, \varphi)$ is an almost contact structure, and $(\mathcal{H}, J)=\left(\operatorname{ker} \eta, \varphi_{\mid \operatorname{ker} \eta}\right)$.

Given an almost $C R$ structure, let $E_{x} \subset T_{x}^{*}(M)$ be the subspace consisting of all pseudohermitian structures on $M$ at $x \in M$. Then $E=\bigcup_{x \in M} E_{x}$ is (the total space of) a real line subbundle of the cotangent bundle $T^{*}(M)$ and the pseudohermitian structures are the globally defined nowhere zero $C^{\infty}$ sections in $E$. If $M$ is oriented then $E$ is trivial i.e., $E \approx M \times \mathbb{R}$ (a vector bundle isomorphism). Therefore $E$ admits globally defined nowhere vanishing sections, equivalently any orientable almost $C R$ manifold admits a 1 -form $\theta$ such that $\operatorname{ker} \theta=\mathcal{H}$ (cf., for example, Ref. [12] Section 1.1.2). On the other hand the existence of a (globally defined) vector field $X_{0}$, transversal to $\mathcal{H}$ at any point is equivalent to the existence of a 1 -form $\theta$ such that $\operatorname{ker} \theta=\mathcal{H}$. In fact, given $\theta$ with $\operatorname{ker} \theta=\mathcal{H}$, we consider a Riemannian metric $g$ on $M$ (which is paracompact) and then define $X_{0}$ by $g\left(X_{0}, X\right)=\theta(X)$ for any vector field $X$. As such, $X_{0}$ is transversal to $\mathcal{H}$ at any point because $\operatorname{ker} \theta=\mathcal{H}$. Hence, by using Proposition 3 , we get the following 
Proposition 4. On an orientable odd-dimensional manifold, the existence of an almost CR structure is equivalent to the existence of an almost contact structure.

Let $(M, \mathcal{H}, J)$ be an almost CR manifold. Put

$$
\mathcal{H}^{1,0}=\{X-i J X: X \in \mathcal{H}\} \text { and } \mathcal{H}^{0,1}=\{X+i J X: X \in \mathcal{H}\}=\overline{\mathcal{H}^{1,0}}, i=\sqrt{-1},
$$

that is, $\mathcal{H}^{1,0}$ (resp. $\mathcal{H}^{0,1}$ ) is the eigenbundle of $J^{C}$ (the $\mathbb{C}$-linear extension of $J$ to $\mathcal{H}^{\mathbb{C}}=\mathcal{H} \otimes \mathbb{C}$ ) corresponding to the eigenvalue $i$ (resp. $-i$ ). Then the complexfication $\mathcal{H}^{\mathbb{C}}$ can be decomposed into direct sum of $( \pm i)$-distributions of $J^{C}$ :

$$
\mathcal{H}^{\mathbb{C}}=\mathcal{H}^{1,0} \oplus \mathcal{H}^{0,1}
$$

Definition 1. An almost $C R$ structure $(M, \mathcal{H}, J)$ is said to be a CR structure on $M$ if $\mathcal{H}^{1,0}$ (and hence also $\mathcal{H}^{0,1}$ ) is (formally) integrable:

$$
\left[\mathcal{H}^{1,0}, \mathcal{H}^{1,0}\right] \subset \mathcal{H}^{1,0}, \text { that is, } Z, W \in C^{\infty}\left(U, \mathcal{H}^{1,0}\right) \text { yields }[Z, W] \in C^{\infty}\left(U, \mathcal{H}^{1,0}\right)
$$

for any open set $U \subset M$.

CR structures are considered mainly from a complex analytical point of view. It is easy to see that an almost $C R$ structure $(M, \mathcal{H}, J)$ is a $C R$ structure if and only if the following two conditions hold:

$$
\begin{gathered}
{[J X, Y]+[X, J Y] \in \mathcal{H}, \quad X, Y \in \mathcal{H},} \\
J\{[J X, Y]+[X, J Y]\}=[J X, J Y]-[X, Y], \quad X, Y \in \mathcal{H} .
\end{gathered}
$$

Of course, if $\operatorname{dim} M=3$ : Any almost $C R$ structure is integrable (in dimension three, the integrability conditions are trivially satisfied). Moreover, if $M$ is a real hypersurface of a complex manifold then the induced almost CR structure is CR (cf. [12], Proposition 1.1). Therefore, an integrable (codimension one) CR structure $(\mathcal{H}, J)$ is often called $C R$ structure of hypersurface type.

Remark 6. Another way to define an almost $C R$ structure is the following. Let $M$ be a real $(2 n+1)$-dimensional manifold. An almost CR structure on $M$ is a complex subbbundle $T^{1,0}(M)$, of complex rank $n$, of the complexified tangent bundle $T(M) \otimes \mathbb{C}$ such that $T^{1,0}(M) \cap T^{0,1}(M)=(0)$ where $T^{0,1}(M)=\overline{T^{1,0}(M)}$ (overbars denote complex conjugates). The integer $n$ is the CR dimension. An almost CR structure $T^{1,0}(M)$ is integrable, and then $T^{1,0}(M)$ is referred to as a CR structure, if $Z, W \in C^{\infty}\left(U, T^{1,0}(M)\right)$ yields $[Z, W] \in C^{\infty}\left(U, T^{1,0}(M)\right)$ for any open set $U \subset M$. The Levi (or maximally complex) distribution is the real rank $2 n$ distribution on $M$ given by $\mathcal{H} \equiv H(M)=\operatorname{Re}\left\{T^{1,0}(M) \oplus T^{0,1}(M)\right\}$. It carries the complex structure

$$
J: \mathcal{H} \rightarrow \mathcal{H}, \quad J(Z+\bar{Z})=i(Z-\bar{Z}), \quad Z \in T^{1,0}(M)(i=\sqrt{-1}) .
$$

Then, $T^{1,0}(M)=\{X-i J X: X \in \mathcal{H}\}=\mathcal{H}^{1,0}$, i.e., $T^{1,0}(M)$ is the eigenbundle of $J^{\mathbb{C}}$ (the $\mathbb{C}$-linear extension of $J$ to $\mathcal{H} \otimes \mathbb{C}$ ) corresponding to the eigenvalue $i$. The pair $(\mathcal{H}, J)$ is the real manifestation of $T^{1,0}(M)$.

Now, we want to compare the normality condition Equation (14) of an almost contact structure with the integrability conditions of the induced almost CR structure. We first give the following proposition.

Proposition 5. Let $(\mathcal{H}, J)$ be an almost $C R$ structure on an odd-dimensional manifold $M$ induced by an almost contact structure $(\eta, \xi, \varphi)$. Then, 
(1) $(\mathcal{H}, J)$ is a $C R$ structure if and only if $S(X, Y)=0$ for any $X, Y \in \mathcal{H}$, where

$$
S(X, Y):=\varphi([J X, Y]+[X, J Y])-[J X, J Y]+[X, Y], \quad X, Y \in \mathcal{H} .
$$

(2) the tensor $h=(1 / 2) \mathcal{L}_{\xi} \varphi$ vanishes if and only if $\mathcal{L}_{\xi} \eta=0$ and $\mathcal{L}_{\xi} J=0$;

(3) if $g$ is a semi-Riemannian metric compatible with the almost contact structure $(\eta, \xi, \varphi)$, then

$$
\mathcal{L}_{\xi} \eta=0 \Longleftrightarrow \nabla_{\xi} \xi=0 \text {, where } \nabla \text { is the Levi-Civita connection. }
$$

Proof. (1) We have to show that the condition $S=0$ is equivalent to the integrability conditions Equations (31) and (32). Since $\varphi^{2}=-I+\eta \otimes \xi$, we have

$$
S(X, Y)=-\varphi^{2}(S(X, Y))+\eta(S(X, Y)) \xi .
$$

So $S(X, Y)=0$ implies $\eta(S(X, Y))=0$. Then, by using $\eta \circ \varphi=0$, we get $\eta([J X, J Y]-[X, Y])=0$ and thus $([J X, J Y]-[X, Y]) \in \mathcal{H}$, that is Equation (31). Replacing $X$ by $J X$, we get $([X, J Y]+[J X, Y]) \in \mathcal{H}$, and so Equation (33) becomes

$$
S(X, Y)=J([J X, Y]+[X, J Y])-[J X, J Y]+[X, Y], \quad X, Y \in \mathcal{H} .
$$

Thus $S(X, Y)=0$ implies the condition Equation (32). Conversely, if Equations (31) and (32) are satisfied, then $S=0$ is trivial.

(2) Recall that $2 h X=\left(\mathcal{L}_{\xi} \varphi\right)(X)=\mathcal{L}_{\xi} \varphi X-\varphi \mathcal{L}_{\xi} X=[\xi, \varphi X]-\varphi[\xi, X]$. So, $h=0$ if and only if $h X=0$ for any $X \in \mathcal{H}$. On the other hand, for $X \in \mathcal{H},\left(\mathcal{L}_{\xi} \eta\right)(X)=-\eta([\xi, X])$, and so $\mathcal{L}_{\xi} \eta=0$ means $[\xi, X] \in \mathcal{H}$. Then, for $X \in \mathcal{H}: h=0$ implies $[\xi, \varphi X]=\varphi[\xi, X] \in \mathcal{H}$, that is, $[\xi, X] \in \mathcal{H}$, and $0=2 h X=[\xi, \varphi X]-\varphi[\xi, X]=[\xi, J X]-J[\xi, X]=\left(\mathcal{L}_{\xi} J\right)(X)$. Conversely, it is not difficult to see that $\mathcal{L}_{\xi} \eta=0$ and $\mathcal{L}_{\xi} J=0$ imply $h=0$.

(3) In such a case, the condition $\mathcal{L}_{\xi \eta}=0$ is equivalent to the condition that $\xi$ is geodesic with respect to the Levi-Civita connection. In fact, for any $X \in \mathcal{H}$ : $\left(\mathcal{L}_{\xi} \eta\right)(X)=0 \Longleftrightarrow g\left(\xi, \nabla_{\xi} X-\nabla_{X} \xi\right)=0$.

Remark 7. An almost contact structure $(\eta, \xi, \varphi)$ satisfying the condition $\xi \in$ ker $d \eta$ is called a natural almost contact structure. This class of almost contact structures has been introduced and studied in the paper [32]. We note that $2 d \eta(\xi, X)=\mathcal{L}_{\xi} \eta$, so the condition that defines this structure is equivalent to the condition $\mathcal{L}_{\xi} \eta=0$ considered in Proposition 5. In particular, any contact semi-Riemannian manifold satisfies the condition $\mathcal{L}_{\xi} \eta=0$, equivalently $\nabla_{\xi} \xi=0$.

S. Ianus (cf. [2], Theorem 6.6 p. 92) proved that a normal almost contact manifold is a CR-manifold. The following Theorem completes this result.

Theorem 11. Let $(\mathcal{H}, J)$ be an almost $C R$ structure on an odd-dimensional manifold $M$ induced by an almost contact structure $(\eta, \xi, \varphi)$. Then, the almost contact structure $(\eta, \xi, \varphi)$ is normal if and only if almost $C R$ structure $(\mathcal{H}, J)$ is integrable and the tensor $h=0$. In particular, if $\mathcal{L}_{\xi} \eta=0,(\eta, \xi, \varphi)$ is normal if and only if $(\mathcal{H}, J)$ is integrable and $\mathcal{L}_{\xi} J=0$. 
Proof. By Proposition 5 we know that $(\mathcal{H}, J)$ is a CR structure if and only the tensor $S$ defined on $\mathcal{H}$ by Equation (33) vanishes. For $X, Y \in \mathcal{H}$ :

$$
\begin{aligned}
{[\varphi, \varphi](X, Y) } & =[J X, J Y]+\varphi^{2}[X, Y]-\varphi([J X, Y]+[X, J Y]) \\
& =[J X, J Y]-[X, Y]+\eta([X, Y]) \xi-\varphi([J X, Y]+[X, J Y]) \\
& =[J X, J Y]-[X, Y]-2 d \eta(X, Y) \xi-\varphi([J X, Y]+[X, J Y]) \\
& =[J X, J Y]-[X, Y]-2 d \eta(X, Y) \xi-\varphi\left(-\varphi^{2}([J X, Y]+[X, J Y])+\eta([J X, Y]+[X, J Y]) \xi\right) \\
& =[J X, J Y]-[X, Y]-2 d \eta(X, Y) \xi+\varphi^{3}([J X, Y]+[X, J Y]) .
\end{aligned}
$$

that is,

$$
[\varphi, \varphi](X, Y)+2 d \eta(X, Y) \xi=-S(X, Y)
$$

Moreover, for $X \in \mathcal{H}$, from

and

$$
[\varphi, \varphi](X, \xi)=\varphi^{2}[X, \xi]-\varphi([\varphi X, \xi])=-[X, \xi]+\eta([X, \xi]) \xi-\varphi([\varphi X, \xi])
$$

we have

$$
2 h \varphi X=\left(\mathcal{L}_{\xi} \varphi\right)(\varphi X)=-[\xi, X]-\varphi[\xi, \varphi X]=[X, \xi]+\varphi[\varphi X, \xi],
$$

$$
[\varphi, \varphi](X, \xi)+2 d \eta(X, \xi) \xi=-2 h \varphi X
$$

Therefore, from Equations (14), (34), and (35) we obtain that $(\eta, \xi, \varphi)$ is normal if and only if almost CR structure $(\mathcal{H}, J)$ is integrable (i.e., $S=0$ ) and the tensor $h=0$. The second part follows from (3) of Proposition 5.

\subsection{Non-Degenerate Almost CR Structures and Contact Semi-Riemannian Structures}

We have already observed that the existence of an almost CR structure $(\mathcal{H}, J)$ on an $(2 n+1)$-dimensional manifold $M$ induced by an almost contact structure is related to the existence of a 1 -form $\theta$ such that $\operatorname{ker} \theta=\mathcal{H}$ (cf. Proposition 3).

Definition 2. A pseudohermitian structure on an almost $C R$ manifold $(M, \mathcal{H}, J)$ is a 1 -form $\theta$ such that $\operatorname{ker} \theta=\mathcal{H}$ and the Levi form $L_{\theta}$, defined by

$$
L_{\theta}(X, Y):=(d \theta)(X, J Y), \quad X, Y \in \mathcal{H}
$$

is Hermitian, that is, $L_{\theta}(J X, J Y)=L_{\theta}(X, Y)$. In such a case, $(\mathcal{H}, J, \theta)$ is called pseudohermitian almost $C R$ structure on $M$.

It should be observed that, for any $X, Y \in \mathcal{H}$, the following are equivalent:

$$
\begin{gathered}
\theta([J X, Y])+\theta([X, J Y])=0 ; \\
(d \theta)(J X, Y)+(d \theta)(X, J Y)=0 \quad\left(\text { i.e., }-L_{\theta}(Y, X)+L_{\theta}(X, Y)=0\right) ; \\
L_{\theta}(J X,-J Y)+L_{\theta}(X, Y)=0 .
\end{gathered}
$$

Then, we have

Proposition 6. Let $(\mathcal{H}, J)$ be an almost $C R$ structure and $\theta$ an 1 -form such that $\operatorname{ker} \theta=\mathcal{H}$. Then, the following properties are equivalent:

(a) $L_{\theta}$ is Hermitian, that is, $(\mathcal{H}, J, \theta)$ is pseudohermitian;

(b) $L_{\theta}$ is symmetric, that is, $L_{\theta}(X, Y)=L_{\theta}(Y, X)$;

(c) the partial integrability condition Equation (31) is satisfied. 
In the case of an almost CR structure induced by an almost contact semi-Riemanian structure, we have:

Proposition 7. Let $(\mathcal{H}=\operatorname{ker} \theta, J)$ be an almost $C R$ structure induced by an almost contact semi-Riemannian structure $(\eta=\theta, \xi, \varphi, g)$. Then, $(\mathcal{H}, J, \theta)$ is a pseudohermitian almost $C R$ structure if and only if the tensor

$$
q:=\varphi \circ \nabla \xi-\nabla \xi \circ \varphi
$$

is symmetric on $\mathcal{H}$, where $\nabla$ is the Levi-Civita connection of $g$. In particular, if $(\eta=\theta, \xi, \varphi, g)$ is a contact semi-Riemannian structure, or an almost $\alpha$-coKähler structure, then $q=2 h=\mathcal{L}_{\xi} \varphi$ and so it is symmetric.

Proof. We show that the partial integrability condition Equation (31) is satisfied if and only if $q$ is symmetric on $\mathcal{H}$.

$$
\begin{aligned}
{[J X, Y]+[X, J Y] } & \in \mathcal{H}, \quad X, Y \in \mathcal{H} \Longleftrightarrow g\left(\nabla_{J X} Y-\nabla_{Y} J X+\nabla_{X} J Y-\nabla_{J Y} X, \xi\right)=0, \quad X, Y \in \mathcal{H} ; \\
& \Longleftrightarrow-g\left(\nabla_{\varphi X} \xi, Y\right)+g\left(\nabla_{Y} \xi, \varphi X\right)-g\left(\nabla_{X} \xi, \varphi Y\right)+g\left(\nabla_{\varphi Y} \xi, X\right)=0, \quad X, Y \in \mathcal{H} ; \\
& \Longleftrightarrow g\left(\varphi \nabla_{X} \xi-\nabla_{\varphi X} \xi, Y\right)=g\left(\varphi \nabla_{Y} \xi-\nabla_{\varphi Y} \xi, X\right), \quad X, Y \in \mathcal{H} ; \\
& \Longleftrightarrow g(q X, Y)=g(X, q Y), \quad X, Y \in \mathcal{H} .
\end{aligned}
$$

If $(\eta=\theta, \xi, \varphi, g)$ is a contact semi-Riemannian structure, $q=2 h$ follows from Equation (4).

If $(\eta=\theta, \xi, \varphi, g)$ is an almost $\alpha$-coKähler structure, $q=2 h$ follows from (3.1) of [33].

Definition 3. A pseudohermitian almost $C R$ structure $(\mathcal{H}, J, \vartheta)$ is said to be a non-degenerate (pseudohermitian) almost $C R$ structure if the Levi form $L_{\theta}$ is, in addition, non-degenerate (equivalently, $\theta$ is a contact form, i.e., $\theta \wedge(d \theta)^{n}$ is a volume form).

In the sequel by a non-degenerate almost CR structure we will mean a non-degenerate pseudohermitian almost CR structure. So, a nondegenerate almost CR structure satisfies the partial integrability condition Equation (31). We remark that two pseudohermitian structures $\theta$ and $\tilde{\theta}$ on the same almost CR manifold, are related by

$$
\tilde{\theta}=\lambda \theta \text { for some } C^{\infty} \text { function } \lambda: M \rightarrow \mathbb{R} \backslash\{0\} .
$$

This gives $d \tilde{\theta}=d \lambda \wedge \theta+\lambda d \theta$, then $L_{\tilde{\theta}}=\lambda L_{\theta}$ and hence non degeneracy is a CR invariant.

Remark 8. In dimension three any non-degenerate almost $C R$ manifold is a $C R$ manifold. If $\operatorname{dim} M>3$, there exist examples of non-degenerate almost $C R$ manifolds which do not satisfy Equation (32), i.e., are not CR manifolds. For example, we have (cf. Theorem 25): If $(M, g)$ is a semi-Riemannian manifold, $\operatorname{dim} M=n \geq 3$, the standard non-degenerate almost $C R$ structure $(\mathcal{H}, J, \theta)$ on the tangent hyperquadric bundle $T_{\mathcal{E}}(M, g)=\left\{(x, u) \in T M \mid g_{x}(u, u)=\varepsilon\right\}, \varepsilon \in\{ \pm 1\}$, is integrable, i.e., it is a non-degenerate CR structure, if and only if $(M, g)$ has constant sectional curvature.

Let $(M, \mathcal{H}, J, \theta)$ be a non-degenerate almost CR manifold. Let us extend $J$ to an endomorphism $\varphi$ of the tangent bundle by requesting that $\varphi=J$ on $\mathcal{H}$ and $\varphi(T)=0(T$ is the Reeb vector field of $\theta)$. Then

$$
\varphi^{2}=-I+\theta \otimes T
$$

and $(\theta, T, \varphi)$ is an almost contact structure. In particular, $\theta \circ \varphi=0$. The Webster metric is the semi-Riemannian metric $g_{\theta}$ defined by

$$
g_{\theta}(X, Y)=L_{\theta}(X, Y)=(d \theta)(X, \varphi Y), g_{\theta}(X, T)=0, g_{\theta}(T, T)=\varepsilon
$$

for any $X, Y \in \mathcal{H}$, where $\varepsilon= \pm 1$. Equivalently,

$$
g_{\theta}=(d \theta)(\cdot, \varphi \cdot)+\varepsilon \theta \otimes \theta .
$$


Since $g_{\theta}(X, \varphi Y)=-(d \theta)(X, Y), \eta=-\theta=\varepsilon g_{\theta}(-T, \cdot)$ and $\varphi^{2}=-I+\theta \otimes T$, then by Equation (1) we get that

$$
\left(\eta=-\theta, \xi=-T, \varphi, g=g_{\theta}\right)
$$

is a contact semi-Riemannian structure on $M$. If we denote by $g_{\theta}^{+}$the Webster metric with $T$ space-like and by $g_{\theta}^{-}$the Webster metric with $T$ time-like, then

$$
g_{\theta}^{+}=g_{\theta}^{-}+2 \theta \otimes \theta
$$

This fact agrees with the change of the causal character of the Reeb vector field (cf. Equation (8)). In particular, if $g_{\theta}^{+}$is Riemannian, then $g_{\theta}^{-}$is Lorentzian with $T$ time-like.

Conversely, a contact semi-Riemannian structure $(\eta, \xi, \varphi, g)$ defines a nondegenerate pseudohermitian almost CR structure given by

$$
\left(\mathcal{H}=\operatorname{ker} \eta, \theta=-\eta, J=\varphi_{\mid \mathcal{H}}\right),
$$

and $L_{\theta}=g_{\mid \mathcal{H}}$ is the corresponding Levi form which is nondegenerate and Hermitian, that is, Equation (31) is satisfied.

If the Levi-form $L_{\theta}$ is positive definite, the Webster metric $g_{\theta}$ (with $\varepsilon=1$ ) is a Riemannian metric and "non-degenerate" is replaced by "strictly pseudo-convex".

Thus, we have the following (cf. also [34], Proposition 2.1)

Proposition 8. The notion of non-degenerate (resp. strictly pseudo-convex) almost CR structure $(\mathcal{H}, J, \theta)$ is equivalent to the notion of contact semi-Riemannian (resp. Riemannian) structure $(\varphi, \xi, \eta, g)$. In particular, non-degenerate (resp. strictly pseudo-convex) CR structures $(\mathcal{H}, J, \theta)$ correspond to contact semi-Riemannian (resp. Riemannian) structures satisfying the condition Equation (32).

\section{- Some remarks}

1. We note that the non-degeneracy is more natural in $C R$ geometry with respect to strictly pseudo-convexity. In fact non-degeneracy is a $C R$ invariant property, i.e., it is invariant under a transformation $\tilde{\theta}=\lambda \theta$, where $\lambda: M \rightarrow R-\{0\}$ is a smooth function, while strictly pseudo-convexity is not a CR invariant property (if $L_{\theta}$ is positive definite and $\tilde{\theta}=-\theta$, then $L_{\tilde{\theta}}$ is negative definite). In particular, if $(\mathcal{H}, \theta, J)$ is a non-degenerate almost $\mathrm{CR}$ structure, then for any real constant $t \neq 0$, $(\mathcal{H}, \tilde{\theta}=t \theta, J)$ is a non-degenerate almost CR structure. Moreover, the Webster metrics $g_{\tilde{\theta}}$ and $g_{\theta}$ are related, taking account that $\tilde{\varphi}=\varphi$, by

that is,

$$
g_{\tilde{\theta}}=(d \tilde{\theta})(\cdot, \tilde{\varphi} \cdot)+\varepsilon \tilde{\theta} \otimes \tilde{\theta}=t(d \theta)(\cdot, \varphi \cdot)+\varepsilon t^{2} \theta \otimes \theta=t\left(g_{\theta}-\varepsilon \theta \otimes \theta\right)+\varepsilon t^{2} \theta \otimes \theta,
$$

$$
g_{\tilde{\theta}}=\operatorname{tg}_{\theta}+\varepsilon t(t-1) \theta \otimes \theta
$$

This is related to the deformation Equation (16).

2. Let $(\mathcal{H}(M), J, \theta)$ be a non-degenerate almost $\mathrm{CR}$ structure and $\left(\eta=-\theta, \xi=-T, \varphi, g=g_{\theta}\right)$ the corresponding contact semi-Riemannian structure. Since

$$
d \theta(X, Y)=d \theta\left(X,-\varphi^{2} Y+\theta(Y) T\right)=d \theta\left(X,-\varphi^{2} Y\right)=-L_{\theta}(X, \varphi Y)=-g_{\theta}(X, \varphi Y)
$$

then we get that $\left(\theta, \bar{\xi}=T, \varphi, \bar{g}=-g_{\theta}\right)$ is still a contact semi-Riemannian structure with $\bar{\varepsilon}=-\varepsilon$. This second structure is obtained by Equation (13), i.e., reversing the first contact semi-Riemannian structure.

3. Let $\eta$ be a contact 1 -form. Then, there exists an associated metric for $\eta$ if and only if there exists an almost complex structure $J$ on $\mathcal{H}=\operatorname{ker} \eta$ such that the Levi form $L_{\eta}=d \eta(\cdot, J \cdot)$ is Hermitian.

A generalization of the basic results in pseudohermitian geometry to the case of a contact Riemannian manifold whose almost CR structure is not integrable was started by S. Tanno [20]. Results in this direction are given also in [35-37]. 
- Hypersurface of an indefinite Kaehler manifold.

The property (1) in Proposition 5 suggests to look the almost contact structure of a hypersurface of an indefinite Kaehler manifold. Let $(\bar{M}, \bar{J}, \bar{g})$ be an indefinite $(2 n+2)$-Kaehler manifold (cf. [38] for definitions and examples). Suppose that $M$ is an orientable non-degenerate real hypersurface of $\bar{M}$. Let $N$ be a normal vector field, $\bar{g}(N, N)=\varepsilon$, that defines the orientation of $M$. Then, the tensors

$$
\xi=-\bar{J} N, \quad g=\bar{g}_{\mid M}, \quad \eta=\varepsilon g(\xi, \cdot), \quad \varphi=\bar{J}-\varepsilon \bar{g}(\bar{J}, N) N,
$$

define an almost contact semi-Riemannian structure. Moreover, we have (see, for example, Ref. [39])

$$
\text { (a) }\left(\nabla_{X} \varphi\right) Y=\eta(Y) A X-\varepsilon g(A X, Y) \xi, \quad(b) \nabla_{X} \xi=\varphi A X,
$$

where $A=-\bar{\nabla} N$ is the shape operator. Now, consider the almost CR structure induced $\left(\mathcal{H}=\operatorname{ker} \eta, J=\varphi_{\mid \mathcal{H}}\right)$. In particular, for $X, Y \in \mathcal{H},([J X, Y]+[X, J Y]) \in \mathcal{H}$ is a consequence of Equation (38)b. In fact,

$$
\begin{aligned}
g([J X, Y]+[X, J Y], \xi) & =g([\varphi X, Y]+[X, \varphi Y], \xi) \\
& =g\left(\nabla_{\varphi X} Y-\nabla_{Y} \varphi X+\nabla_{X} \varphi Y-\nabla_{\varphi Y} X, \xi\right) \\
& =-g\left(\nabla_{\varphi X} \xi, Y\right)+g\left(\nabla_{Y} \xi, \varphi X\right)-g\left(\nabla_{X} \xi, \varphi Y\right)+g\left(\nabla_{\varphi Y} \xi, X\right) \\
& =-g(\varphi A \varphi X, Y)+g(\varphi A Y, \varphi X)-g(\varphi A X, \varphi Y)+g(\varphi A \varphi Y, X) \\
& =g(A \varphi X, \varphi Y)+g(A Y, X)-g(A X, Y)-g(A \varphi Y, \varphi X) \\
& =0 .
\end{aligned}
$$

Next, since $([J X, Y]+[X, J Y]) \in \mathcal{H}$, from Equation (33) we have

$$
\begin{aligned}
S(X, Y) & =\varphi([\varphi X, Y]+[X, \varphi Y])-[\varphi X, \varphi Y]+[X, Y] \\
& =-\left(\nabla_{\varphi X} \varphi\right) Y+\left(\nabla_{\varphi Y} \varphi\right) X-\left(\nabla_{X} \varphi\right) \varphi Y+\left(\nabla_{Y} \varphi\right) \varphi X .
\end{aligned}
$$

On the other hand, by Equation (38)a, we have $\left(\nabla_{X} \varphi\right) Y=-\varepsilon g(A X, Y) \xi$ for any $X, Y \in \mathcal{H}$, and so we get $S(X, Y)=0$ for any $X, Y \in \mathcal{H}$. Therefore, by 1$)$ of Proposition 5 , the almost $C R$ structure $(\mathcal{H}, J)$ is integrable. So, we proved the following

Proposition 9. Let $(\bar{M}, \bar{J}, \bar{g})$ be an indefinite Kaehler manifold. Suppose that $M$ is an orientable non-degenerate real hypersurface of $\bar{M}$. Then, the almost contact semi-Riemannian structure on $M$ given by (37) defines a $C R$ structure $(\mathcal{H}, J)$ on $M$.

Now, we see when the almost contact semi-Riemannian structure defined by (37) is Sasakian. Suppose that this structure is Sasakian. Then, comparing Equation (15) with Equation (38)a, we get

$$
\eta(Y) A X-\varepsilon g(A X, Y) \xi=g(X, Y) \xi-\varepsilon \eta(Y) X
$$

and taking $Y=\xi$, we have

$$
A X=\eta(A X) \xi+\varepsilon \eta(X) \xi-\varepsilon X .
$$

In particular, $A \xi=\eta(A \xi) \xi$. Then, $\eta(A X)=\varepsilon g(A X, \xi)=\varepsilon g(X, A \xi)=\varepsilon g(X, \eta(A \xi) \xi)=\eta(A \xi) \eta(X)$, and so from Equation (39) we obtain

$$
A=-\varepsilon I+\alpha \eta \otimes \xi, \quad \alpha=\eta(A \xi)+\varepsilon .
$$

Conversely, if $A$ is given by Equation (40), by Equation (38)a we get Equation (15). Then we get the following (cf. [39], and [2] Theorem 6.15 in the Riemannian case). 
Theorem 12. Let $(\bar{M}, \bar{J}, \bar{g})$ be an indefinite Kaehler manifold. Suppose that $M$ is an orientable non-degenerate hypersurface of $\bar{M}$. Then, the almost contact semi-Riemannian structure on $M$ given by Equation (37) is Sasakian if and only if the shape operator is given by Equation (40).

By Proposition 9, the standard pseudohermitian almost CR structure $(\mathcal{H}, \theta, J)$ of an orientable non-degenerate real hypersurface, that is, the one induced by Equation (37), is integrable. Then, Proposition 6 gives that $(\mathcal{H}, \theta, J)$ is always a pseudohermitian CR structure. Moreover, by using Equation (38)b, i.e., $\nabla \xi=\varphi A$, we have

$$
\left.2 d \eta(X, Y)=-\eta[X, Y]=-\varepsilon g\left(\xi, \nabla_{X} Y-\nabla_{Y} X\right)=\varepsilon g(\varphi A+A \varphi) X, Y\right), \quad X, Y \in \mathcal{H}
$$

Consequently, the condition $d \eta=g(\cdot, \varphi)$ is satisfied if and only if

$$
\varphi A+A \varphi=-2 \varepsilon \varphi
$$

Then, we have the following (cf. [2], Theorem 4.12, for the Riemannian case)

Theorem 13. Let $M$ be an orientable non-degenerate real hypersurface of an indefinite Kaehler manifold $\bar{M}$. Then, the almost $C R$ structure $(\mathcal{H}, \theta, J)$ induced on $M$ is always a pseudohermitian $C R$ structure. Moreover, it is a non-degenerate CR structure if and only if the shape operator satisfies Equation (42).

\section{- Levi-flatness}

The "opposite" of Levi non-degenerate is the following definition.

Definition 4. A pseudohermitian almost $C R$ structure $(\mathcal{H}, J, \theta)$ is said to be Levi-flat, or Levi-degenerate, if the Levi form $L_{\theta}$ vanishes.

In particular, an almost contact structure $(\eta, \xi, \varphi)$ on an odd-dimensional manifold $M$, with $d \eta=0$, defines a pseusdohermitian Levi-flat almost CR structure $\left(\mathcal{H}=\operatorname{ker} \eta, \theta=\eta, J=\varphi_{\mid \mathcal{H}}\right)$. If $(M, \mathcal{H}, J, \vartheta)$ is a Levi-flat pseudohermitian almost CR manifold, then $d \theta$ is zero on $\mathcal{H}$ and the Frobenius Theorem shows that $\mathcal{H}$ defines a foliation.

Examples of Levi-flat $C R$ manifolds. First we recall some definitions which are related to the Levi-flat manifolds. An almost $\alpha$-coKähler structure is an almost contact metric structure $(\eta, \xi, \varphi, g)$ such that $d \eta=0$ and $d \Phi=2 \alpha \eta \wedge \Phi$ for some real constant $\alpha$, where $\Phi=g(\cdot, \varphi)$ is the fundamental 2 -form (see [33] and the references there in). In particular, $\alpha=0$ gives an almost coKähler structure (equivalently, an almost cosymplectic structure following Goldberg-Yano [40]) and $\alpha \neq 0$ gives an almost $\alpha$-Kenmotsu structure. In any case, an almost $\alpha$-coKähler structure defines a Levi-flat pseudohermitian almost CR manifold.

In the case of an orientable non-degenerate real hypersurface of an indefinite Kaehler manifold $\bar{M}$, by using Equation (41), the standard pseudohermitian CR structure $(\mathcal{H}, \theta, J)$ of $M$ is Levi-flat, i.e., $L_{\theta}=0$, if and only if $\varphi A=-A \varphi$ on $\mathcal{H}$. On the other hand, if we consider the fundamental 2-form $\Phi$, we have $(d \Phi)(X, Y, Z)=0$ (cf. [39]). Hence, an orientable non-degenerate real hypersurface of an indefinite Kähler manifold $\bar{M}$ is almost coKähler if and only if $\varphi A=-A \varphi$.

Recently in the paper [33], see also [41], we proved that an orientable Riemannian three-manifold $(M, g)$ admits an almost $\alpha$-coKähler structure with $g$ as a compatible metric if and only if $M$ admits a foliation, defined by a unit closed 1-form, of constant mean curvature. Then, in the same paper we show that a simply connected homogeneous almost $\alpha$-coKähler three-manifold is either a Riemannian product of type $\mathbb{R} \times \mathbb{S}^{2}\left(k^{2}\right)$, equipped with its standard coKähler structure, or it is a semidirect product Lie group $G=\mathbb{R}^{2} \rtimes_{A} \mathbb{R}$ equipped with a left invariant almost $\alpha$-coKähler structure. All the three-manifolds listed in this classification are examples of Levi-flat pseudohermitian CR three-manifolds. 


\section{- The embeddability}

A natural difference between the class of $C R$ manifolds and the class of almost CR manifolds is the question of embeddability. In fact, a question of principal interest in the theory of compact, $(2 n+1)$-dimensional CR-manifolds is to understand when a given strictly pseudo-convex CR-structure can be realized by an embedding in $\mathbb{C}^{m}$. This question is only of interest in the three-dimensional case because a theorem of Boutet de Monvel [42] states that any strictly pseudo-convex CR-structure, on a compact $(2 n+1)$-manifold, is realizable as an embedding in some $\mathbb{C}^{m}$, provided $n>1$.

The global embedding problem in CR geometry in dimension 3 has received a lot of attention. In [43], Burns and Epstein considered perturbations of the standard CR structure on the three-sphere $\mathbb{S}^{3}$. They showed that a generic perturbation is non-embeddable and gave a sufficient condition for embeddability ([43], Theorem 5.3). In the same paper, they introduced the notion of stability for CR embeddings. Then Lempert [44] considered the problem of stability of CR embeddings of a compact three-dimensional $C R$ manifold into $\mathbb{C}^{2}$, and proved that if a compact strictly pseudo-convex $C R$ manifold admits a $\mathrm{CR}$ embedding into $\mathbb{C}^{2}$ then this embedding is stable.

S. Chanillo, H. Chiu and P. Yang $([45,46])$ discussed the relationship between the embeddability of three- dimensional closed strictly pseudo-convex CR manifolds and the positivity of the CR Paneitz operator and the CR Yamabe constant. In particular, they proved the embeddability into $\mathbb{C}^{n}$ for some $n$ when the CR Paneitz operator is non-negative and the CR Yamabe constant is positive.

\subsection{The (Generalized) Tanaka-Webster Connection and the Pseudohermitian Torsion}

Let $(M, \mathcal{H}, J, \theta)$ be a non-degenerate almost CR manifold and $\left(\eta=-\theta, \xi=-T, \varphi, g=g_{\theta}\right)$ the associated contact semi-Riemannian structure. The most convenient linear connection for studying $(M, \mathcal{H}, J, \theta)$ is the so-called (generalized) Tanaka-Webster connection $\hat{\nabla}$. This is the linear connection given by

$$
\hat{\nabla}_{X} Y=\nabla_{X} Y+\varepsilon \eta(X) \varphi Y-\eta(Y) \nabla_{X} \xi+\left\{\left(\nabla_{X} \eta\right) Y\right\} \xi
$$

for any $X, Y \in \mathfrak{X}(M)$, where $\nabla$ is the Levi-Civita connection of $g_{\theta}$. Equivalently, $\hat{\nabla}$ is defined by

$$
\hat{\nabla}_{\xi} Y=\nabla_{\xi} Y+\varepsilon \varphi Y, \quad \hat{\nabla}_{X} Y=\pi\left(\nabla_{X} Y\right) \text { for } X, Y \in \mathcal{H}, \quad \hat{\nabla} \xi=0,
$$

where $\pi$ is the usual projection $\pi: T M \rightarrow \mathcal{H}$. The generalized Tanaka-Webster connection $\hat{\nabla}$ is due to Tanno [20] (though confined to the positive definite case). For a nondegenerate almost CR manifold, $\hat{\nabla}$ was considered in $[47,48]$. $\hat{\nabla}$ admits an axiomatic description similar to that of the ordinary Tanaka-Webster connection (cf. Tanaka [10]) except for the property $\hat{\nabla} \varphi=0$. More precisely, $\hat{\nabla}$ is the unique linear connection obeying to the axioms

$$
\begin{gathered}
\hat{\nabla} \eta=0, \quad \hat{\nabla} \xi=0, \quad \hat{\nabla} g=0, \\
\hat{T}(\xi, \varphi X)+\varphi \hat{T}(\xi, X)=0, \quad X \in \mathfrak{X}(M), \\
\hat{T}(X, Y)=2(d \eta)(X, Y) \xi, \quad X, Y \in \mathcal{H}=\operatorname{ker} \eta, \\
\left(\hat{\nabla}_{X} \varphi\right) Y=Q(Y, X), \quad X, Y \in \mathfrak{X}(M) .
\end{gathered}
$$

Here $\hat{T}(X, Y)=\hat{\nabla}_{X} Y-\hat{\nabla}_{Y} X-[X, Y]$ is the torsion tensor field of $\hat{\nabla}$, and $Q$ is the Tanno tensor, i.e.,

$$
Q(Y, X)=\left(\nabla_{X} \varphi\right) Y+\left\{\left(\nabla_{X} \eta\right) \varphi Y\right\} \xi+\eta(Y) \varphi\left(\nabla_{X} \xi\right) .
$$

We note that $Q(\xi, X)=Q(Y, \xi)=0$ and $Q(Y, X)=\left(\nabla_{X} \varphi\right) Y-\eta\left(\nabla_{X} \varphi Y\right) \xi$ for any $X, Y \in \mathcal{H}$. Then, by the same proof given in [20], $Q=0$ if and only if $(\mathcal{H}, J)$ is integrable, that is:

$$
\hat{\nabla} \varphi=0 \Longleftrightarrow(\mathcal{H}, J) \text { is a CR structure, }
$$

and then $\hat{\nabla}$ is the ordinary Tanaka-Webster connection. 
The pseudohermitian torsion of $\hat{\nabla}$ (introduced by Webster in the integrable case [11], see also [12], p. 26) is the vector valued 1 -form $\tau$ on $M$ defined by

and thus

$$
\tau X:=\hat{T}(T, X),
$$

$$
\tau=-\hat{T}(\xi, \cdot)=\ldots=\varphi h=-h \varphi, \text { equivalently: } h=\tau \varphi=-\varphi \tau,
$$

and

$$
\hat{T}=2(\tau \wedge \eta+\mathrm{d} \eta \otimes \xi)
$$

Then, by using

$$
\mathcal{L}_{\xi} g=2 g(\cdot, h \varphi \cdot)=-2 g(\cdot, \tau \cdot)=-2 g(\tau \cdot, \cdot),
$$

(2) of Proposition 5 and Theorem 11, one gets:

Theorem 14. Let $(M, \mathcal{H}, J, \theta)$ be a non-degenerate almost $C R$ manifold. Then,

- $\quad$ the Reeb vector field $\xi$ is Killing with respect to Webster metric $g_{\theta}$ if and only if pseudohermitian torsion $\tau$ vanishes, equivalently $\mathcal{L}_{\xi} J=0$;

- the almost contact structure $(\xi, \varphi, \eta)$ is normal, equivalently the Webster metric $g_{\theta}$ is Sasakian, if and only if the almost $C R$ structure is integrable and the pseudohermitian torsion $\tau$ vanishes;

- a non-degenerate CR manifold is Sasakian if and only if $\mathcal{L}_{\tilde{\xi}} J=0$.

Next, we recall that given a semi-Riemannian manifold $(\bar{M}, \bar{g})$, with $\bar{\nabla}$ the Levi-Civita connection, and a smooth nondegenerate distribution $\mathcal{D}: x \mapsto \mathcal{D}_{x} \subset T_{x} \bar{M}$ on $\bar{M}$, then $\mathcal{D}$ is called minimal distribution if $\operatorname{trace}_{\bar{g}}(B)=0$, where

$$
B(X, Y)=\left(\bar{\nabla}_{X} Y\right)^{\perp} \text { for any } X, Y \in \mathcal{D},
$$

and $\left(\bar{\nabla}_{X} Y\right)^{\perp}$ is the natural projection on $\mathcal{D}^{\perp}$. Moreover, the distribution $\mathcal{D}$ is called totally geodesic if the symmetrized second fundamental form $B_{S}(X, Y):=(1 / 2)(B(X, Y)+B(Y, X))$ vanishes.

Consider the non-degenerate almost $C R$ manifold $(M, \mathcal{H}(M), J, \theta)$. For the Levi distribution $\mathcal{H}(M)$, the second fundamental form $B(X, Y)$ is given by $B(X, Y)=\varepsilon g\left(\nabla_{X} Y, \xi\right) \xi$, and by using Equation (4) we get

$$
\begin{aligned}
\operatorname{trace}_{g}(B) & =\varepsilon \sum_{i=1}^{2 n} \varepsilon_{i} g\left(\nabla_{E_{i}} E_{i}, \xi\right) \xi=-\varepsilon \sum_{i=1}^{2 n} \varepsilon_{i} g\left(\nabla_{E_{i}} \xi, E_{i}\right) \xi \\
& =-\varepsilon \sum_{i=1}^{2 n} \varepsilon_{i} g\left(-\varepsilon \varphi E_{i}-\varphi h E_{i}, E_{i}\right) \xi=\varepsilon\left(\operatorname{trace}_{g} \varphi h\right) \xi,
\end{aligned}
$$

where $E_{i}$ is a local orthonormal basis. Since trace $g(\varphi h)=0$, we get trace $g(B)=0$. Moreover, by using Equation (4), the symmetrized second fundamental form is given by

$$
\begin{aligned}
B_{S}(X, Y) & =(\varepsilon / 2)\left\{g\left(\nabla_{X} Y, \xi\right)+g\left(\nabla_{Y} X, \xi\right)\right\} \xi=-(\varepsilon / 2)\left\{g\left(\nabla_{X} \xi, Y\right)+g\left(\nabla_{Y} \xi, X\right)\right\} \xi \\
& =\varepsilon g(\varphi h X, Y) \xi=-\varepsilon g(\tau X, Y) \xi .
\end{aligned}
$$

Then, we get

Proposition 10. ([34]) For any non-degenerate almost $C R$ manifold $(M, \mathcal{H}, J, \theta)$, the Levi distribution $\mathcal{H}(M)$ is minimal in $\left(M, g_{\theta}\right)$, and it is totally geodesic if and only if the pseudohermitian torsion $\tau$ vanishes.

Now, we give some properties related to the pseudohermitian curvature of a non-degenerate almost $\mathrm{CR}$ manifold $(M, \mathcal{H}, J, \theta)$. Denote by $\hat{R}$ the pseudohermitian curvature tensor, that is, the curvature tensor associated to the generalized Tanaka-Webster connection $\hat{\nabla}$. Then, following Tanno [20,49], the pseudohermitian Ricci tensor $\hat{R} i c$ and the pseudohermitian scalar curvature $\hat{r}$ are defined by 


$$
\hat{R} i c(X, Z)=\operatorname{Tr}_{g} \hat{R}(X, \cdot) Z, \quad \hat{r}=\operatorname{Tr}_{g} \hat{R} i c .
$$

The pseudohermitian scalar curvature $\hat{r}$ is also called the (generalized) Tanaka-Webster scalar curvature [20]. In [48] we considered the following

Definition 5. A nondegenerate almost $C R$ manifold $(M, \mathcal{H}, J, \theta), \operatorname{dim} M=(2 n+1)$, is said to be pseudo-Einstein if the pseudohermitian Ricci tensor $\hat{R} i c$ is proportional to the Levi form, that is, $\hat{R} i c_{\mid \mathcal{H}}=\lambda L_{\theta}$, where $\lambda=\hat{r} / 2 n$.

In the case of a non-degenerate $\mathrm{CR}$ manifold with vanishing pseudohermitian torsion our definition of pseudo-Einstein structure coincides with the definition of J.M. Lee [50]. In general, the pseudo-Einstein condition does not imply that the pseudohermitian scalar curvature is constant, so such a structure is less rigid than an Einstein structure on a semi-Riemannian manifold [50]. Next, we show that this notion is related to the notion of $\eta$-Einstein contact semi-Riemannian manifold given in Section 2.2.

Consider a non-degenerate almost CR manifold $(M, \mathcal{H}, J, \theta), \operatorname{dim} M=(2 n+1)$, with pseudohermitian torsion $\tau=0$. In this case, Equation (43) gives

and in particular

$$
\hat{\nabla}_{X} Y=\nabla_{X} Y+\varepsilon \eta(X) \varphi Y+\varepsilon \eta(Y) \varphi X-g(\varphi X, Y) \xi
$$

$$
\hat{\nabla}_{X \xi}=0, \quad \hat{\nabla}_{\xi} Y=\nabla_{\xi} Y+\varepsilon \varphi Y .
$$

Then, the pseudohermitian Ricci tensor $\hat{R} i c$ and the Tanaka-Webster scalar curvature $\hat{r}$ are given by

$$
\begin{aligned}
\hat{R} i c(\xi, \xi)=\hat{R} i c(X, \xi)=0, \hat{R} i c(X, Y) & =\operatorname{Ric}(X, Y)+2 \varepsilon L_{\theta}(X, Y), X, Y \in \mathcal{H}, \\
\hat{r} & =r+2 n \varepsilon,
\end{aligned}
$$

where Ric and $r$ denote the Ricci tensor and the scalar curvature of the Webster metric $g_{\theta}$. So, we get

Proposition 11. ([48]) Let $(M, \mathcal{H}, J, \theta)$ be a non-degenerate almost $C R$ manifold with pseudohermitian torsion $\tau=0$. Then, the structure $(\mathcal{H}, J, \theta)$ is pseudo-Einstein if and only if the corresponding semi-Riemannian contact structure is $\eta$-Einstein.

When the pseudohermitian torsion $\tau \neq 0$, there are other conditions on $\tau$ with an interesting meaning. Given an oriented, compact, contact manifold $(M, \eta)$, denote by $\mathcal{M}(\eta)$ the set of all Riemannian metrics associated to the contact form $\eta$ and by $\mathcal{A}(\eta)$ the set of all almost CR structures $J$ for which the Levi form is positive definite. By Proposition 8, the sets $\mathcal{M}(\eta)$ and $\mathcal{A}(\eta)$ can be identified.

- The condition $\hat{\nabla}_{\xi} \tau=0$. Tanno [20] considered the Dirichlet energy

$$
E(g)=\int_{M}\left\|\mathcal{L}_{\xi} g\right\|^{2} d v
$$

defined for any $g \in \mathcal{M}(\eta)$. Then, he found the critical point condition ([20], Theorem 5.1)

$$
\nabla_{\xi} \mathcal{L}_{\xi} g=2\left(\mathcal{L}_{\xi} g\right) \varphi \text {, equivalently } \nabla_{\xi} h=-2 \varphi h .
$$

We note that this condition has a tensorial character, so it holds also in the non compact case. The Dirichlet energy Equation (50) was studied by Chern and Hamilton [51] for compact contact three-manifolds as a functional defined on the set $\mathcal{A}(\eta)$ (there was an error in their calculation of the critical point condition, as was pointed out by Tanno). Moreover, since $\operatorname{Ric}(\xi, \xi)=2 n-t r h^{2}=2 n-\left\|\mathcal{L}_{\xi} g\right\|^{2} / 4$, the functional Equation (50) is equivalent to the 
functional $L(g)=\int_{M} \operatorname{Ric}(\xi, \xi) d v$ studied in general dimension, for compact regular contact manifold, by Blair ([2], Section 10.3).

Now, since $\mathcal{L}_{\xi} g=2 g(\varphi \cdot, h \cdot)=-2 g(\tau \cdot, \cdot)$, where $g=g_{\theta}$ and $\tau=\varphi h$ is the pseudohermitian torsion, we have

$$
\left\|\mathcal{L}_{\xi} g\right\|^{2}=4\|\tau\|^{2}
$$

Then, to consider the Dirichlet energy Equation (50) is equivalent to consider the following

$$
E(g)=\int_{M}\|\tau\|^{2} d v
$$

defined on the set $\mathcal{A}(\eta)$. Moreover, using the Tanaka-Webster connection given by Equation (43), we get

$$
\hat{\nabla}_{\xi} \tau=\varphi \hat{\nabla}_{\xi} h=\nabla_{\xi} \tau+2 \varepsilon \varphi \tau \text {, equivalently } \hat{\nabla}_{\xi} h=\nabla_{\xi} h+2 \varepsilon \varphi h, \text { where } \varepsilon=1 .
$$

Thus, the critical point condition Equation (51) becomes

$$
\hat{\nabla}_{\tilde{\xi}} \tau=0
$$

If $T_{1} M$ is the tangent sphere bundle of a Riemannian manifold of constant curvature -1 , equipped with its standard contact structure. Then, the standard associated Riemannian metric (cf. Section 5.1) satisfies the critical point condition $\hat{\nabla}_{\xi} \tau=0$, equivalently $\nabla_{\xi} h=-2 \varphi h$ (cf. proof of Theorem 10.13 in [2]). Moreover, we recall the following (see [15,52]):

Theorem 15. Let $(M, \eta, g)$ be a compact H-contact $(2 n+1)$-manifold such that $\hat{\nabla}_{\xi} \tau=0$, i.e., $g$ is critical for the Dirichlet energy $E(g)$. If Ric $+c g$ is positive definite for some constant $c<2-2\|\tau\| / \sqrt{2 n}$, then the first Betti number of $M$ vanishes.

- The condition $\hat{\nabla}_{\xi} \tau=2 \varepsilon \varphi \tau$. This condition, equivalently $\nabla_{\xi} \tau=0$, or also $\nabla_{\xi} h=0$, is related to some interesting property. It is equivalent to the curvature property [34]:

the $\xi$-sectional curvatures $K(\xi, X)$ and $K(\xi, \varphi X)$ are equal for any $X \in \mathcal{H}$,

where if $X_{p}$ is null, $K\left(\xi_{p}, X_{p}\right)$ is a lightlike sectional curvature (cf., for example, Ref. [53] p. 95). Moreover, if $(M, \eta, g)$ is an oriented, compact contact Riemannian three-manifold, the condition $\nabla_{\xi} \tau=0$ (equivalently $\hat{\nabla}_{\xi} \tau=2 \varphi \tau$ ) is the critical point condition for the functional

$$
I(g)=\int_{M} r d v \text { defined on the set } \mathcal{M}(\eta),
$$

where $r$ is the Riemannian scalar curvature [54]. If $T_{1} M$ is the tangent sphere bundle of a flat Riemannian manifold, equipped with its standard contact structure, then the standard associated Riemannian metric (cf. Section 5.1), satisfies the critical point condition $\hat{\nabla}_{\xi} \tau=2 \varphi \tau$, equivalently $\nabla_{\xi} h=0$ (cf. Theorem 9.7 in [2]).

Recall that if $M$ is an oriented compact manifold, by a classical result of Hilbert (see also Nagano [55]), a Riemannian metric $g$ on $M$ is a critical point of the integral of the scalar curvature, $I(g)=\int_{M} r d v$, as a functional on the set of all Riemannian metrics of the same total volume on $M$, if and only if $g$ is an Einstein metric. Now, by using a result of [54], we get that a contact Riemannian three-manifold is $\eta$-Einstein if and only if it is $H$-contact and satisfies the critical point condition $\hat{\nabla}_{\xi} \tau=2 \varphi \tau$ (equivalently, $\nabla_{\xi} \tau=0$ ).

- The Chern-Hamilton functional. In CR geometry a natural functional is the the integral of the generalized Tanaka-Webster scalar curvature. For a strictly pseudo-convexity almost CR manifold, 
i.e., for a contact Riemannian manifold, the generalized Tanaka-Webster scalar curvature $\hat{r}$ is given by (cf. [20])

$$
\hat{r}=r-\operatorname{Ric}(\xi, \xi)+4 n
$$

This is eight times the Webster scalar curvature $W$ as defined by Chern and Hamilton [51] on three-dimensional contact manifolds. In the same paper, Chern and Hamilton proved, in dimension three, that the critical point condition for the functional

$$
I_{w}(g)=\int_{M} \hat{r} d v \quad \text { defined on the } \operatorname{set} \mathcal{A}(\eta),
$$

is the vanishing of the pseudohermitian torsion $\tau$. An alternate proof of this important result was given by the present author [54]. Tanno [20] studied the functional $I_{w}(g)$ in arbitrary dimension.

- An interpretation of the Tanaka-Webster scalar curvature. Recall that a contact form $\eta$ on a compact manifold $M$ is called regular if its Reeb vector field $\xi$ is regular, i.e., any point of $M$ has a neighborhood such that any integral curve of $\xi$ passing through the neighborhood passes through only once. In this case $M$ is a principal $\mathbb{S}^{1}$-bundle over a symplectic manifold $B$ whose fundamental 2-form $\Omega$ has integral periods (a Hodge manifold). The corresponding fibration $p: M \rightarrow B=M$ is known as the Boothby-Wang fibration [56]. Now, let $(M, \eta, g)$ be a compact simply connected regular Sasakian, $(2 n+1)$-manifold. Then, the base of the Boothby-Wang fibration is a compact Kähler manifold of complex dimension $n$, with Kähler metric $\tilde{g}$ and fundamental 2-form $\Omega$ satisfying (cf., for example, Ref. [57,58])

$$
g=p^{*} \tilde{g}+\eta \otimes \eta, \quad d \eta=p^{*} \Omega .
$$

Moreover, the scalar curvatures $r, \tilde{r}$ of $(M, g)$ and $(B, \tilde{g})$, respectively, are related by

$$
\tilde{r}=r+2 n .
$$

On the other hand, in the Sasakian case, Equation (53) becomes

$$
\hat{r}=r+2 n .
$$

So, in this case, the Tanaka-Webster scalar curvature $\hat{r}$ is the scalar curvature $\tilde{r}$ of the Kähler manifold $(B, \tilde{g})$ base of the Boothby-Wang fibration. We note that a compact simply connected homogeneous Sasakian manifold is regular [57].

\subsection{Contact Geometry of CR Manifolds}

In this subsection we give a presentation of some results about the study of CR manifolds, i.e., the $\mathrm{CR}$ integrable case, from the point of view of contact geometry.

- The Olszak's result in the semi-Riemannian setting

First rigidity results concerning non-degenerate almost CR manifold with the Webster metric of constant curvature were obtained in the Riemannian case by D.E. Blair and Z. Olszak. Blair [59] showed that a contact form does not admit any flat associated Riemannian metric in dimension $\geq 5$. Then, Olszak [60] generalizing this result proved that if a contact Riemannian $(2 n+1)$-manifold, $n \geq 2$, is of constant curvature $\kappa$, then the manifold is Sasakian and $\kappa=1$. In the semi-Riemannian case, we have $([7,8])$

Theorem 16. Let $(M, \eta, g)$ be a contact semi-Riemannian $(2 n+1)$-manifold. If $n \geq 2$ and $(M, g)$ is of constant sectional curvature $\kappa$, then $\kappa=\varepsilon=g(\xi, \xi)$ and $h^{2}=0$.

In terms of CR geometry, Theorem 16 becomes 
Theorem 17. Let $(M, \mathcal{H}, J, \theta)$ be a $(2 n+1)$-dimensional non-degenerate almost $C R$ manifold, $n \geq 2$. If the Webster metric $g_{\theta}$ is of constant sectional curvature $\kappa$, then $\kappa=\varepsilon=g_{\theta}(\xi, \xi)$ and the pseudohermitian torsion satisfies $\tau^{2}=0$.

In particular, since $\varepsilon= \pm 1$, a non-degenerate almost CR structure does not admit any flat semi-Riemannian Webster metric in dimension $\geq 5$, so Blair's result also holds in the semi-Riemannian setting. But, there are examples of non-degenerate almost $C R$ manifold with $\tau^{2}=0$ and $\tau \neq 0$. In fact we have the following (see [34] for details).

Example 5. Consider the space $M=\mathbb{R}^{5}\left(x_{1}, x_{2}, x_{3}, x_{4}, z\right)$ and two smooth functions $\alpha, \beta \in C^{\infty}\left(\mathbb{R}^{5}\right)$. We put $\partial_{i}=\frac{\partial}{\partial x_{i}}, i=1,2,3,4$, and $\partial_{z}=\frac{\partial}{\partial z}$. Define the vector fields $X_{i},=1, \ldots, 5$ on $M$ by

$$
\begin{aligned}
& \sqrt{2} X_{1}=\alpha \partial_{1}+\partial_{2}+\partial_{3}-2 x_{1} \partial_{z}, \quad \sqrt{2} X_{2}=\partial_{1}-\beta \partial_{2}-\partial_{4}+2 x_{2} \partial_{z}, \\
& \sqrt{2} X_{3}=-\alpha \partial_{1}+\partial_{2}-\partial_{3}+2 x_{1} \partial_{z}, \quad \sqrt{2} X_{4}=\partial_{1}+\beta \partial_{2}+\partial_{4}-2 x_{2} \partial_{z}, \quad X_{5}=\partial_{z} .
\end{aligned}
$$

Moreover, we define the 1-form $\eta=2 x_{1} \mathrm{~d} x_{3}+2 x_{2} \mathrm{~d} x_{4}+\mathrm{d} z$, the vector field $\xi=X_{5}=\partial_{z}$, the tensor $\varphi$ by

$$
\varphi\left(X_{1}\right)=-X_{2}, \quad \varphi\left(X_{2}\right)=X_{1}, \quad \varphi\left(X_{3}\right)=-X_{4}, \quad \varphi\left(X_{4}\right)=X_{3}, \quad \varphi\left(X_{5}\right)=0
$$

and the semi-Riemannian metric $g$ of signature $(-,-,+,+, \pm)$ by

$$
g\left(X_{1}, X_{1}\right)=g\left(X_{2}, X_{2}\right)=-1, \quad g\left(X_{3}, X_{3}\right)=g\left(X_{4}, X_{4}\right)=1, \quad g\left(X_{i}, X_{j}\right)=0, i \neq j, \quad g\left(X_{5}, X_{5}\right)=\varepsilon= \pm 1 .
$$

Then $(\xi, \varphi, \eta, g)$ defines a contact semi-Riemannian structure, and so a non-degenerate almost CR structure on $M$, with Levi distribution $\mathcal{H}=\operatorname{ker} \eta=\operatorname{span}\left(X_{1}, X_{2}, X_{3}, X_{4}\right)$. Moreover, we can construct a frame $\left\{E_{1}, E_{2}, E_{3}, E_{4}, \xi\right\}$ of vector fields on $\mathbb{R}^{5}$ with $E_{i} \in \mathcal{H}$ null vector fields which satisfy

$$
\tau E_{1}=0, \quad \tau E_{2}=0, \quad \tau E_{3}=-\frac{\partial_{z}(\beta-\alpha)}{2} E_{1}, \quad \tau E_{4}=\frac{\partial_{z}(\beta-\alpha)}{2} E_{2}
$$

Therefore, $\tau^{2}=0$. Moreover, $\tau=0$ if and only if $\partial_{z}(\beta-\alpha)=0$. So, taking the functions $\alpha, \beta$ such that $\partial_{z} \beta \neq \partial_{z} \alpha$, we obtain a non-degenerate almost $C R$ structure with $\tau^{2}=0$ and $\tau \neq 0$. Moreover, this structure in general is not a $C R$ structure. In fact, taking for example $X=E_{1}$ and $Y=E_{3}$, one gets that the integrability condition Equation (32) is satisfied if and only if $\left(\partial_{1}(\beta+\alpha), \partial_{2}(\beta-\alpha)\right)=(0,0)$.

If the almost CR structure is integrable, we get the Olszak's result in the semi-Riemannian setting. In fact, we have the following.

Theorem 18. ([34]) Let $(M, \mathcal{H}, J, \theta)$ be a non-degenerate $C R$ manifold, $\operatorname{dim} M=2 n+1, n \geq 2$. If the Webster metric $g_{\theta}$ is of constant sectional curvature $\kappa$, then $\kappa=\varepsilon=g_{\theta}(\xi, \xi)$ and the pseudohermitian torsion $\tau=0$, i.e., the Webster metric is Sasakian.

Remark 9. In dimension three, a non-degenerate CR manifold with the Webster metric $g_{\theta}$ locally symmetric (in particular, of constant sectional curvature) is either flat or of constant sectional curvature $\kappa=\varepsilon=g(\xi, \xi)$, and in the second case the metric is Sasakian [7].

- $\quad(\kappa, \mu)$-spaces

We recall that a contact metric manifold $(M, \eta, \xi, \varphi, g)$ is said to be a $(\kappa, \mu)$-space if its structure satisfies the $(k, \mu)$-nullity condition, that is, the curvature tensor satisfies

$$
R(X, Y) \xi=\kappa(\eta(X) Y-\eta(Y) X)+\mu(\eta(X) h Y-\eta(Y) h X)
$$

for some $\kappa, \mu \in \mathbb{R}$. This condition, which is a generalization of the Sasakian condition Equation (23), defines an interesting class of contact metric manifolds introduced by Blair, Koufogiorgos and 
Papantoniou [61]. We note that this class is invariant for $\mathcal{D}$-deformations. A classification Theorem of the $(\kappa, \mu)$-spaces is given by E. Boechx [62]. Moreover, we recall the following (cf. [2], p. 124)

Theorem 19. $A(\kappa, \mu)$-space $M$ is a strictly pseudoconvex $C R$-manifold. Moreover, $\kappa \leq 1$, and if $\kappa=1$ the pseudohermitian torsion vanishes and thus the structure is Sasakian. If $\kappa<1$, the $(\kappa, \mu)$ condition determines the curvature of $M$ completely.

In particular, the Ricci operator $Q$ and the scalar curvature $r$ of a $(2 n+1)$-dimensional $(\kappa, \mu)$-space $M, \kappa<1$, are given by

$$
\begin{gathered}
Q X=(2(n-1)-n \mu) X+(2(n-1)+\mu) h X+(n(2 \kappa+\mu)-2(n-1)) \eta(X) \xi, \\
r=2 n(2(n-1)+\kappa-n \mu) .
\end{gathered}
$$

Then, $(\kappa, \mu)$-spaces are examples of $H$-contact manifolds. For a non-Sasakian $(\kappa, \mu)$ space, Boeckx Boechx [62] introduced an invariant

$$
I_{M}=\frac{2-\mu}{2 \sqrt{1-\kappa}}
$$

and showed that for two non-Sasakian $(\kappa, \mu)$ spaces $M, M^{\prime}$, we have $I_{M}=I_{M^{\prime}}$ if and only if up to a $\mathcal{D}$-homothetic deformation, the two spaces are locally isometric as contact metric manifolds.

- $\quad$ Sasakian geometry by using a variational theory

In paper [63], Barletta and Dragomir built a variational theory of geodesics of the Tanaka-Webster connection $\hat{\nabla}$ on a strictly pseudoconvex CR manifold $M$. They obtained the first and second variation formulae for the Riemannian length of a curve in $M$ and showed, in particular, that in general geodesics of $\hat{\nabla}$ admitting horizontally conjugate points do not realize the Riemannian distance. The paper also contained interesting results concerning the pseudohermitian sectional curvature $K_{\theta}$, that is, the sectional curvature defined by the tensor

$$
\hat{R}(X, Y, Z, W)=g_{\theta}(R(X, Y) Z, W),
$$

where $\hat{R}(X, Y) Z$ is the pseudohermitian curvature tensor associated to the Tanaka-Webster connection $\hat{\nabla}$, and $g_{\theta}$ is the Webster metric. For example they proved (cf. Theorems 4 and 5 of [63]) the following.

Theorem 20. Let $M$ be a a strictly pseudoconvex CR manifold.

(1) If $M$ has non-positive pseudohermitian sectional curvature, then it has no horizontally conjugate points.

(2) If $M$, of $C R$ dimension $n>1$, has constant pseudohermitian sectional curvature, then it has vanishing pseudohermitian torsion $(\tau=0)$ if and only if the Tanaka-Webster connection of $M$ is flat.

- Almost contact structures belonging to a CR structure

Let $(\mathcal{H}, J)$ be a $C R$ structure on a odd-dimensional manifold $M$. We say that an almost contact structure $(\theta, \xi, \varphi)$ belongs to the $C R$ structure $(\mathcal{H}, J)$ if $\operatorname{ker} \theta=\mathcal{H}$ and $J=\varphi_{\mid \mathcal{H}}$. Then, by Lemma 1.1 of [64], two almost contact structures $(\theta, \xi, \varphi)$ and $\left(\theta^{\prime}, \xi^{\prime}, \varphi^{\prime}\right)$ belong to the same CR structure $(\mathcal{H}, J)$ if and only if

$$
\theta^{\prime}=\varepsilon e^{\lambda} \theta, \quad \xi^{\prime}=\varepsilon e^{-\lambda}\left(\varphi X_{0}+\xi\right), \quad \varphi^{\prime}=\varphi+\theta \otimes X_{0}
$$

for some smooth function $\lambda$ and vector field $X_{0} \in \mathcal{H}$, where $\varepsilon= \pm 1$.

Denote by $(\theta, \xi, \varphi)^{*}$ an almost contact structure belongs to a non-degenerate CR structure $(\mathcal{H}, J)$ and satisfying the condition $[\xi, \mathcal{H}] \subset \mathcal{H}$. Then, K.Sakamoto and Y. Takemura [64] proved the existence of a unique linear connection associated to $(\theta, \xi, \varphi)^{*}$. Moreover in [65], they obtained a curvature 
invariant of the pseudo-conformal geometry, that is, a tensor field invariant under the change of almost contact structures belonging to the same non-degenerate CR-structure. For the case of a normal almost contact structure the invariant tensor field is just the Bochner curvature tensor.

\section{Homogeneous Non-Degenerate CR Three-Manifolds}

The main purpose of this Section is to give a presentation of some results about homogeneous non-degenerate CR three-manifolds.

\subsection{The Classification Theorem}

Recall some definitions about the homogeneity.

A contact manifold $(M, \eta)$ is said to be homogeneous if there exists a (connected) Lie group $G$ of diffeomorphisms acting transitively on $M$ and leaving $\eta$ invariant. A contact semi-Riemannian manifold $(M, \eta, g)$ is said to be homogeneous if there exists a (connected) Lie group $G$ of isometries acting transitively on $M$ and leaving $\eta$ invariant, that is, for any $x, y \in M$ there exists $f \in G$ such that $y=f(x)$, and $f^{*} \eta=\eta$ for every $f \in G$.

A $C^{\infty}$ map $f: M \rightarrow M^{\prime}$ between almost CR manifolds is a CR map if

$$
f_{* x}\left(\mathcal{H}_{x}\right) \subset \mathcal{H}_{f(x)}^{\prime} \quad \text { and } \quad f_{* x} \circ J_{x}=J_{f(x)}^{\prime} \circ f_{* x}
$$

In particular a CR transformation is a diffeomorphism $f$ such that

$$
f_{* x}\left(\mathcal{H}_{x}\right)=\mathcal{H}_{f(x)}^{\prime} \quad \text { and } \quad f_{* x} \circ J_{x}=J_{f(x)}^{\prime} \circ f_{* x}
$$

for any $x \in M$. If $M=M^{\prime}$ a CR transformation is also called $C R$ automorphism.

Remark 10. Typical examples of $C R$ maps are got as traces of holomorphic maps of Kaehlerian manifolds on real hypersurfaces. Precisely, let $\bar{M}$ be a Kaehlerian manifold. Any orientable real hypersurface $M \subset \bar{M}$ admits a natural $C R$ structure (cf. Proposition 9). If $M^{\prime} \subset \bar{M}^{\prime}$ is another oriented real hypersurface in the Kaehlerian manifold $\bar{M}^{\prime}$ and $F: \bar{M} \rightarrow \bar{M}^{\prime}$ is a holomorphic map such that $F(M) \subset M^{\prime}$ then $\left.f \equiv F\right|_{M}: M \rightarrow M^{\prime}$ is a CR map. The statements above hold true for traces of holomorphic maps among indefinite Kaehlerian manifolds [47]. A characterization of K-contact structures in terms of CR maps is presented in Theorem 32 of this paper.

Let $\theta$ and $\theta^{\prime}$ be pseudohermitian structures on the almost CR manifolds $M$ and $M^{\prime}$ respectively. If $f: M \rightarrow M^{\prime}$ is a CR map, then $f^{*} \theta^{\prime}=\mu \theta$ for some $\mu \in C^{\infty}(M)$. In particular, a CR map $f$ is called pseudohermitian map if $\mu=c$ for some $c \in \mathbb{R}$. Also $f$ is isopseudohermitian if $c=1$.

Let $(M, \mathcal{H}, \theta, J)$ be a pseudohermitian almost CR manifold. Denote by $\mathcal{P}_{s h}(M, \theta)$ the group of all CR automorphisms $f: M \rightarrow M$ such that $f^{*} \theta=\theta$.

In general the group $\mathcal{P}_{s h}(M, \theta)$ is not a Lie group, but if $M$ is a pseudohermitian, or a non-degenerate, CR manifold then $\mathcal{P}_{s h}(M, \theta)$ is a Lie group (Ref. [12], p. 60; Ref. [66], p. 218) of dimension $\leq(n+1)^{2}$, where $(2 n+1)=\operatorname{dim} M$.

A non-degenerate, or pseudohermitian, CR manifold $(M, \mathcal{H}, \theta, J)$ is said to be homogeneous non-degenerate, or pseudohermitian, $C R$ manifold if there exists a Lie group $G \subseteq \mathcal{P}_{\text {sh }}(M, \theta)$ acting transitively on $M$ (cf. [12] p. 341), that is, for any $x, y \in M$ there exists $f \in G$ such that $y=f(x)$, and

$$
f^{*} \theta=\theta, \quad f_{* x} \circ J_{x}=J_{f(x)} \circ f_{* x} \quad \text { for every } f \in G .
$$

If $(M, \eta, \xi, \varphi, g)$ is a contact semi-Riemannian manifod and $f$ a diffeomorphism of $M$, then

$$
\text { (a) } f^{*} \eta=\eta \Rightarrow f_{*} \xi=\xi ; \quad(b) f^{*} \eta=\eta \text { and } f^{*} g=g \Rightarrow f_{* x} \circ \varphi_{x}=\varphi_{f(x)} \circ f_{* x} .
$$

Note that two contact semi-Riemannian manifolds $(M, \eta, g, \xi, \varphi),,\left(M^{\prime}, \eta^{\prime}, g^{\prime}, \xi^{\prime}, \varphi^{\prime}\right)$ are called isomorphic (or equivalent) if there exists a isometry $f:(M, g) \rightarrow\left(M^{\prime}, g^{\prime}\right)$ such that

$$
f^{*} \eta^{\prime}=\eta, \quad f_{*} \xi=\xi^{\prime} \text { and } f_{*} \circ \varphi=\varphi^{\prime} \circ f_{*} .
$$

By using Equation (56), we get the following 
Proposition 12. Let $(M, \mathcal{H}, \theta, J)$ a non-degenerate $C R$ manifold and $\left(\eta=-\theta, \xi=-T, \varphi, g=g_{\theta}\right)$ the corresponding contact semi-Riemannian structure. Then,

$(M, \mathcal{H}, \theta, J)$ is homogeneous if and only if $(M, \eta, \xi, \varphi, g)$ is homogeneous.

In other words,

$$
f^{*} \theta=\theta \text { and } f_{* x} \circ J_{x}=J_{f(x)} \circ f_{* x} \text { if and only if } f^{*} \eta=\eta \text { and } f^{*} g=g .
$$

Recall that there is a canonical way to associate a contact Riemannian structure to a contact Lorentzian structure (and conversely). If $\left(\eta, \xi, \varphi, g_{L}\right)$ is a contact Lorentzian structure on a smooth manifold $M, \operatorname{dim} M=2 n+1$, where the Reeb vector field $\xi$ is time-like, then (cf. Section 2.2 and also Equation (36))

$$
\left(\eta, \xi, \varphi, g=g_{L}+2 \eta \otimes \eta\right)
$$

is a contact Riemannian structure on $M$. The scalar curvatures $r_{R}$ and $r_{L}$ of $g$ and $g_{L}$ are related by Equation (20):

$$
r_{L}=r_{R}+4 n+2 \operatorname{tr} h^{2}
$$

Now, let $(M, \mathcal{H}, \theta, J)$ be a non-degenerate $C R$ three-manifold. Then, the Levi form $L_{\theta}$ is definite, and we can assume $L_{\theta}$ positive definite (if necessary, we change $\theta$ with $-\theta$ ). Therefore, without loss in generality, in dimension three, we can consider either $g_{\theta}$ Lorentzian with $T$ time-like or $g_{\theta}$ Riemannian. In particular, the Sasakian condition $\tau=0$ does not depend on the causal character of the Reeb vector field T. Moreover, for a non-degenerate CR three-manifold, the Tanaka-Webster scalar curvature is given by

$$
\hat{r}=r+\varepsilon\left(2+\operatorname{tr} h^{2}\right), \quad \text { where } \varepsilon=g_{\theta}(T, T) .
$$

So, the Tanaka-Webster scalar curvature $\hat{r}$ does not depend on the causal character of the Reeb vector field $T$, i.e., $\hat{r}_{L}=\hat{r}_{R}$. In fact,

$$
\hat{r}_{L}=r_{L}-\left(2+\operatorname{tr} h^{2}\right)=r_{R}+4+2 \operatorname{tr} h^{2}-\left(2+\operatorname{tr} h^{2}\right)=\hat{r}_{R} .
$$

If we consider the scalar torsion $\left\|\mathcal{L}_{\xi} g_{\theta}\right\|$ introduced by Chern and Hamilton in [51] in their study of contact Riemannian three-manifolds, since $\mathcal{L}_{\xi} g_{\theta}=2 g_{\theta}(\varphi \cdot, h \cdot)=-2 g_{\theta}(\tau \cdot, \cdot)$, we have

$$
\left\|\mathcal{L}_{\xi} g_{\theta}\right\|^{2}=4\|\tau\|^{2}=4 t r h^{2},
$$

and thus

$$
\hat{r}=r+2+t r h^{2}=r+2+\|\tau\|^{2}=8 W,
$$

where $W$ is the Webster scalar curvature as defined by Chern and Hamilton [51].

Since the Webster scalar curvature $W$ and the scalar pseudohermitian torsion $\|\tau\|$ do not depend on the causal character of the Reeb vector field $T$, that is, they depend only on Levi form $L_{\theta}$, then it is natural to consider these invariants in order to classify the homogeneous nondegenerate $C R$ three-manifolds. More precisely, we consider the invariant $W$ in the Sasakian case, and the invariant

$$
p:=\frac{2 \sqrt{2} W}{\|\tau\|}
$$

in the non Sasakian case. Then, the classification Theorem of [67], can be reformulated in the following form.

Theorem 21. A simply connected, homogeneous, non-degenerate $C R$ three-manifold $(M, \mathcal{H}, \theta, J)$ is a Lie group $G$ equipped with a left-invariant non-degenerate CR structure. More precisely, one of the following cases occurs: 
- $\quad$ Sasakian case (i.e., the pseudohermitian torsion $\tau=0$ ).

(1) If $G$ is unimodular, then it is

(i) the Heisenberg group $H^{3}$ when $W=0$;

(ii) the 3-sphere group $S U(2)$, i.e., the special unitary group, when $W>0$;

(iii) $\widetilde{S L}(2, \mathbb{R})$, the universal covering of the special linear group $S L(2, \mathbb{R})$, when $W<0$;

(2) If $G$ is non-unimodular, then its Lie algebra is given by

$$
\left[e_{1}, e_{2}\right]=\alpha e_{2}+2 \xi, \quad\left[e_{1}, \xi\right]=\left[e_{2}, \xi\right]=0,
$$

where $\alpha \neq 0$. In this case, $W=-\alpha^{2} / 4<0$.

- $\quad$ Non Sasakian case (i.e., the pseudohermitian torsion $\tau \neq 0$ ).

(1) If $G$ is unimodular, then it is

(i) $\operatorname{SU}(2)$ when $p>1$;

(ii) $\widetilde{E}(2)$, universal covering of the Lie group of orientation-preserving rigid motions of Euclidean plane, when $p=1$;

(iii) $E(1,1)$, the group of rigid motions of Minkowski plane, when $p=-1$;

(iv) $\widetilde{S L}(2, R)$ when $-1 \neq p<1$.

(2) If $G$ is non-unimodular, then its Lie algebra is given by

$$
\left[e_{1}, e_{2}\right]=\alpha e_{2}+2 \xi, \quad\left[e_{1}, \xi\right]=\gamma e_{2}, \quad\left[e_{2}, \xi\right]=0
$$

where $\alpha, \gamma \neq 0$. In this case, $W=-\left(\alpha^{2}+\gamma\right) / 4,\|\tau\|=(1 / \sqrt{2})|\gamma|$ and thus $p<1$.

Remark 11. In [67] we used the notation $\tau$ to denote $\mathcal{L}_{\xi} g_{\theta}$. Moreover, in the same paper, in the statement of Theorem 21 we used the Riemannian Webster metric $g_{\theta}$.

The structure on the unimodular Lie groups in Theorem 21 satisfy, in the Riemannian case, the $(\kappa, \mu)$-nullity condition Equation (54), that is, they are $(\kappa, \mu)$-space. Besides, we note that in the non Sasakian case the role played by the invariant $p$ defined by Equation (57). Since, $W=(2-\mu) / 4$ and

$$
\begin{aligned}
\left\|\mathcal{L}_{\xi} g\right\|^{2}=8(1-\kappa), \text { i.e., }\|\tau\|=\frac{\left\|\mathcal{L}_{\xi g}\right\|}{2} & =\sqrt{2} \sqrt{1-\kappa} \text {, then } \\
& p=\frac{2 \sqrt{2} W}{\|\tau\|}=\frac{2-\mu}{2 \sqrt{1-\kappa}}
\end{aligned}
$$

which is the invariant $I_{M}$ of Boeckx defined by Equation (55).

\subsection{Consequences of the Classification Theorem}

In this subsection we examine some interesting consequences of the classification Theorem. A first immediate consequence is the following

Corollary 3. The 3-sphere group SU(2) is the only simply connected 3-manifold which admits a homogeneous non-degenerate $C R$ structure with Webster scalar curvature $W>(1 / 2 \sqrt{2})\|\tau\|$.

In particular, the sphere $\mathbb{S}^{3}$ is the only simply connected 3-manifold which admits a homogeneous Sasakian structure with Webster scalar curvature $W>0(W=1$ for the standard Sasakian structure).

Moreover, examining the proof of Theorem 21, more precisely by using (3.3) and (3.10) of [67], we get 
Proposition 13. The Lie group $\widetilde{E}(2)$ is the only simply connected 3-manifold which admits a homogeneous non-degenerate CR structure with flat Riemannian Webster metric. In such a case $W=+1 / 2$.

In the Lorentzian case, one gets

Proposition 14. ([7]) The Lie group $E(1,1)$ is the only simply connected 3-manifold which admits a homogeneous non-degenerate CR structure with flat Lorentzian Webster metric. In such a case $W=-1 / 2$.

H. Geiges [68] proved that a compact 3-manifold admits a Sasakian structure if and only if it is diffeomorphic to a left invariant quotient of $S U(2)$, the Heisenberg group $H^{3}$ or $\widetilde{S} L(2, \mathbb{R})$ by a discrete group. As a consequence of Theorem 21 we have

Proposition 15. The unimodular Lie groups $S U(2)$, the Heisenberg group $H^{3}, \widetilde{S} L(2, \mathbb{R})$, and the non-unimodular Lie group with Lie algebra defined by Equation (58), are the only simply connected three-manifolds which admit a homogeneous Sasakian structure.

Now, let $(\eta, g)$ be a homogeneous Sasakian structure on the sphere $\mathbb{S}^{3}$, with Webster scalar curvature $W>0$. Since $W=(r+2) / 8>0$, then $\tilde{\eta}=t \eta$ and $\tilde{g}=t g+t(t-1) \eta \otimes \eta$, for $t=W$, define a Sasakian structure on $\mathbb{S}^{3}$ with $\tilde{g}$ of constant sectional curvature +1 (cf. [52], Section 3). In particular, $(\tilde{\eta}, \tilde{g})$ is isomorphic to the standard Sasakian structure $\left(\eta_{0}, g_{0}\right)$ [4]. Then, we can assume $(\tilde{\eta}, \tilde{g})=\left(\eta_{0}, g_{0}\right)$, and consequently we have

$$
g=\frac{1}{t} g_{0}+\frac{(1-t)}{t^{2}} t \eta \otimes t \eta=\frac{1}{t}\left(g_{0}+\left(\frac{1}{t}-1\right) \eta_{0} \otimes \eta_{0}\right)=a g_{a}
$$

where $g_{a}=g_{0}+(a-1) \eta_{0} \otimes \eta_{0}, a=1 / W>0$, is a Berger metric, that is, a metric defined as the canonical variation $g_{a}, a>0$, of the standard metric $g_{0}$ on $\mathbb{S}^{3}$, obtained deforming $g_{0}$ along the fibres of the Hopf fibration:

$$
g_{a \mid \xi_{0}^{\perp}}=g_{0 \mid \xi_{0}^{\perp}}, \quad g_{a}\left(\xi_{0}, \cdot\right)=a g_{0}\left(\xi_{0}, \cdot\right)=a \eta_{0}, \quad g_{a}\left(\xi_{0}, \xi_{0}^{\perp}\right)=0,
$$

where $\xi_{0}$ denotes the standard Hopf vector field on $\mathbb{S}^{3}$. Therefore we get:

Proposition 16. In the second part of Corollary 3 , the Sasakian metric on $\mathbb{S}^{3}$ is homothetic to a Berger metric.

Remark 12. The main result of [51] says that any contact structure on a compact and orientable three-manifold has a contact form and a contact Riemannian metric whose Webster scalar curvature $W$ is either a constant $\leq 0$ or is everywhere strictly positive. Now, if $M$ is a compact Sasakian 3-manifold with Webster scalar curvature $W>0$, then $M$ admits a contact Riemannian structure of positive Ricci curvature [69]. If, in addition, $M$ is simply connected, by a deep result of R.S. Hamilton [70], $M$ is diffeomorphic to the sphere $\mathbb{S}^{3}$. However, this fact is not too surprising since a compact simply connected manifold which admits a nonsingular Killing vector field is diffeomorphic to $\mathbb{S}^{3}$ (cf. [52] Section 4).

By the proof of Theorem 21 , the Lie algebra of the lie group $\widetilde{S L}(2, R)$ is defined by

$$
\left[e_{2}, e_{3}\right]=2 \xi, \quad\left[e_{3}, \xi\right]=\lambda_{2} e_{2}, \quad\left[\xi, e_{2}\right]=\lambda_{3} e_{3},
$$

where for $\left(\lambda_{2}, \lambda_{3}\right)$ we have the following possibilities: $(-,-),(-,+)$, and $(+,-)$. Moreover the Webster scalar curvature is given by $W=\left(\lambda_{2}+\lambda_{3}\right) / 4$. So, for $\lambda_{3}=-\lambda_{2}$ we get $W=0$. For the non-unimodular Lie group with Lie algebra defined by Equation (59), the the Webster scalar curvature is given by $W=-\left(\alpha^{2}+\gamma\right) / 4$ and so $W=0$ for $\gamma=-\alpha^{2}$. Then, we get the following (which corrects Corollary 3.3 of [67]).

Corollary 4. The Heisenberg group $H^{3}, \widetilde{S L}(2, R)$ and the non-unimodular Lie group with its Lie algebra defined by Equation (59), are the only simply connected 3-manifolds which admit a homogeneous nondegenerate 
$C R$ structure with Webster scalar curvature $W=0$. In particular, the Heisenberg group $H^{3}$ is the only simply connected three-manifold which admits a non-degenerate $C R$ structure with pseudohermitian torsion $\tau=0$ and Webster scalar curvature $W=0$.

In Theorem 21, if we consider $g_{\theta}$ Lorentzian and denote by $r_{L}$ the corresponding scalar curvature, then in the Sasakian case (i.e., when $\tau=0$ ), the conditions $W=0, W>0, W<0$, and $W=-\alpha^{2} / 4$ are equivalent to $r_{L}=2, r_{L}>2, r_{L}<2$, and $r_{L}=-2 \alpha^{2}+2<2$, respectively. On the other hand, for a Lorentzian Sasakian three-manifold, when $r_{L}<2$, the Lorentzian $K$-contact structure $(\widetilde{\eta}, \tilde{g})$ obtained by a $\mathcal{D}$-homothetic deformation in correspondence to $t=\left(2-r_{L}\right) / 8=-W$ is Einstein (see Section 2.2), and so of constant sectional curvature -1 . Therefore, we get the following corollary which does not have a Riemannian counterpart.

Corollary 5. The unimodular Lie group $\widetilde{S L}(2, R)$ and the non-unimodular Lie group with Lie algebra defined by Equation (58), are the only simply connected three-manifolds which admit a homogeneous Lorentzian-Sasakian structure of constant sectional curvature $\kappa=-1$.

\section{- Homogeneous bi-contact metric three-manifolds}

We close this subsection with a very short presentation of a recent notion introduced by the present author in [71]. H. Geiges and J. Gonzalo ([72,73]) introduced and studied the notion of taut contact circle on a three-manifold, that is, a pair of contact forms $\left(\eta_{1}, \eta_{2}\right)$ such that the 1 -forms $\eta_{a}=a_{1} \eta_{1}+a_{2} \eta_{2}$ are contact forms with the same volume form for all $a \in \mathbb{S}^{1}$. In the paper [71] we introduce a Riemannian approach to the study of taut contact circles on three-manifolds. A natural related notion, that we introduce, is the one of taut contact metric circle $\left(\eta_{1}, \eta_{2}, g\right)$, that is, $\left(\eta_{1}, \eta_{2}\right)$ is a taut contact circle and $g$ is a Riemannian metric associated to both the contact forms $\eta_{1}$ and $\eta_{2}$. More in general, we introduce the notion of bi-contact metric structure $\left(\eta_{1}, \eta_{2}, g\right)$, where $\left(\eta_{1}, \eta_{2}\right)$ is a pair of arbitrary contact forms and $g$ is a Riemannian metric associated to both the contact forms $\eta_{1}$ and $\eta_{2}$ such that the same contact forms are orthogonal with respect to $g$, i.e., the corresponding Reeb vector fields $\xi_{1}, \xi_{2}$ are orthogonal. On the other hand, in the classical definition of three-contact metric structure $\left(\eta_{1}, \eta_{2}, \eta_{3}, g\right)$, also called contact metric three-structure, we have three contact forms and a Riemannian metric $g$ associated to the three contact forms, satisfying additional conditions that imply the orthogonality of the three forms with respect to $g$ (see, for example, Ref. [2] Chapter 14 and [3] Chapter 13).

Moreover, a three-contact metric structure is three-Sasakian (see, for example, Ref. [2] p. 293, Theorem 14.1), and a three-Sasakian three-manifold is of constant sectional curvature +1 (see, for example, Ref. [2] p. 294, Theorem 14.3). So, our definition of bi-contact metric structure seems more appropriate, at least in dimension three, in the sense that it is very less rigid. In particular, we characterize the existence of a taut contact metric circle and of a bi-contact metric structure on a three-manifold. Note that a taut contact metric circle is a bi-contact metric structure, but the converse is not true. Then, we give a complete classification of simply connected three-manifolds which admit a bi-H-contact metric structure. In particular, by using the classification given in Theorem 21 , we get (cf. [71], Corollary 4.7).

Theorem 22. A simply connected three-manifold admits a homogeneous bi-contact metric structure if and only if it is diffeomorphic to one of the following Lie groups: $\operatorname{SU}(2), \widetilde{S L}(2, \mathbb{R}), \widetilde{E}(2), E(1,1)$.

\subsection{Some Results in Arbitrary Dimension}

Now we briefly recall some results, in arbitrary dimension, about contact homogeneity and spherical CR manifolds.

- D. E. Blair (see [2], p. 120) conjectured the non-existence of contact Riemannian manifolds having non positive sectional curvature, with the exception of the flat 3-dimensional case. In this direction, 
A. Lotta [74] got the following (as a consequence of a more general theorem and by using the classification given in Theorem 21).

Theorem 23. The only simply connected homogeneous contact Riemannian $(2 n+1)$-manifold having non-positive sectional curvature is the Lie group $\widetilde{E}(2)$ endowed with a flat left invariant contact Riemannian structure.

- A contact Riemannian manifold is said to be a strongly locally $\varphi$-symmetric space if the reflections in the integral curves of the Reeb vector field are isometries. Examples of strongly locally $\varphi$-symmetric spaces include the non- Sasakian $(\kappa, \mu)$-manifolds (see [2], p. 146; more in general we refer to [2] Section 7.9 for a discussion on weakly and strongly locally $\varphi$-symmetric spaces). Boeckx and Cho in the paper [75] proved the following

Theorem 24. Let $M$ be a locally homogeneous contact Riemannian $(2 n+1)$-manifold. If $M$ is strongly locally $\varphi$-symmetric, then it is a $(\kappa, \mu)$-space.

- Recently E.M. Correa [76] gives a new study on compact, $(2 n+1)$-dimensional, homogeneous contact manifolds. More precisely, this paper contains:

a description of contact structure for any compact homogeneous contact manifold;

a description of G-invariant Sasaki-Einstein structure for any compact homogeneous contact manifold;

a description of Calabi-Yau metrics on cones with compact homogeneous Sasaki-Einstein manifolds as link of isolated singularity;

a description of crepant resolution of Calabi-Yau cones with certain compact homogeneous Sasaki-Einstein manifolds as link of isolated singularity.

This study of homogeneous contact manifolds is based on the Kähler geometry of complex flag manifolds.

- The present author and L. Vanhecke [77] proved that a compact, simply connected, five-dimensional, homogeneous contact manifold $M$ is diffeomorphic to $\mathbb{S}^{5}$ or $\mathbb{S}^{2} \times \mathbb{S}^{3}$. In both cases the underlying homogeneous contact metric structure is Sasakian (and hence is a CR structure). This result is based on the fact that the contact structure is regular and the base $B$ of the Boothby-Wang fibration $\pi: M \rightarrow B$ is a compact simply connected homogeneous Kähler manifold of complex dimension two. In general, we note that every compact simply connected homogeneous contact manifold is a homogeneous Sasaki-Einstein manifold (Ref. [76], Remark 2.17).

- D. V. Alekseevsky and A. Spiro [66,78] gave a classification of all compact, simply connected, $(2 n+1)$-dimensional, homogeneous non-degenerate CR manifolds $M$. This classification is based on a description of the maximal connected compact group of automorphisms of $M$.

- $\quad$ CR manifolds which are locally CR equivalent to the unit sphere $\mathbb{S}^{2 n+1}$, endowed with the standard CR structure as a real hypersurface of $\mathbb{C}^{n+1}$, are called spherical $C R$ manifolds. In particular, non-degenerate $C R$ manifolds with a vanishing Chern pseudoconformal curvature tensor are spherical ([12], p. 61). If $M$ is a spherical CR manifold, Burns and Shnider ([79], Section 1) defined a development map $f: \tilde{M} \rightarrow \mathbb{S}^{2 n+1}$, where $\tilde{M}$ is its universal cover. Moreover, they proved that if the group of $C R$ automorphisms is transitive on $M$, then $f: \tilde{M} \rightarrow \mathbb{S}^{2 n+1}$ is a covering and $f(\tilde{M})$ is homogeneous domain $\mathcal{D}$ in $\mathbb{S}^{2 n+1}$. Thus to classify the simply connected spherical homogeneous CR manifolds it suffices to classify homogeneous domain in $\mathbb{S}^{2 n+1}$ ([79], Theorem 3.1). In particular, in dimension three, we have a list of five examples ([79], p. 229):

$$
\mathcal{D}=\mathbb{S}^{3}=\left\{(z, w) \in \mathbb{C}^{2}:|z|^{2}+|w|^{2}=1\right\} ;
$$


(2) $\mathcal{D}=\mathbb{S}^{3}-\{(z, 0)\}$;

(3) $\mathcal{D}=\mathbb{S}^{3}-\{(1,0)\}$;

(4) $\mathcal{D}=\mathbb{S}^{3}-\left(\mathbb{S}^{3} \cap \mathbb{R}^{3}\right)$;

(5) $\mathcal{D}=\left\{(z, w) \in \mathbb{C}^{2}: \operatorname{Im} w=|z|^{2}, \operatorname{Im} z>0\right\}$.

We note that domain (5) does not admit any compact quotients ([79], Proposition 5.5).

- R. Lehmann and D. Feldmueller [80] proved that the only CR-structure (of hypersurface type) on $\mathbb{S}^{2 n+1}, n>1$, which admits a transitive action of a Lie group of CR-transformations is the standard CR-structure. For $\mathbb{S}^{3}$ all possible homogeneous $\mathrm{CR}$-structures of hypersurface type are classified in [81] (cf. also [80], p. 524).

- G. Dileo and A. Lotta [82] studied spherical symmetric CR manifolds. A strictly pseudo-convex CR manifold $M$ is said to be $C R$-symmetric if for each point $x \in M$ there exists a CR-isometry $\sigma: M \rightarrow M$ such that $\sigma(x)=x$ and $(d \sigma)_{x \mid \mathcal{H}_{x}}=-I_{d}$. In particular, they proved the following. Let $M$ be a strictly pseudo-convex CR manifold, $\operatorname{dim} M>3$, with pseudohermitian torsion $\tau \neq 0$. Then, $M$ is locally CR-symmetric if and only if the underlying contact metric structure $(\eta, \xi, \varphi, g)$ satisfies the $(k, \mu)$-nullity condition, that is, the curvature tensor satisfies Equation (54). In such a case $M$ is spherical if and only if the Webster scalar curvature vanishes.

\section{Geometry of Tangent Hyperquadric Bundles}

The geometry of the unit tangent sphere bundle $T_{1} M$ of a Riemannian manifold $(M, g)$ equipped with the Sasaki metric, and in particular with the standard contact Riemannian structure, has been studied by many authors. A motivation of this study depends of the fact that often properties of $T_{1} M$ characterize the base manifold (see, for example, Blair's book [2] Chapter 9, and from the point of view of the CR geometry Tanno [83]).

If $(M, g)$ is a semi-Riemannian manifold of index $v>0$, the Sasaki metric induced on tangent hyperquadrics bundle $T_{\varepsilon} M, \varepsilon= \pm 1$, is a semi-Riemannian metric of index $2 v-1$ if $\varepsilon=-1$ and the index is $2 v$ if $\varepsilon=+1$. In such case we have few results about the the geometry of $T_{\varepsilon} M$ (see [84], Ref. [85] and more recently [48]). In this Section we discuss some results of [48] on the geometry of $T_{\varepsilon} M$ equipped with the standard non-degenerate almost $C R$ structure.

\subsection{The Standard Non-Degenerate Amost $C R$ Structure on $T_{\varepsilon} M$}

Let $(M, g)$ be a semi-Riemannian manifold of index $v, 0 \leq v \leq n=\operatorname{dim} M$. At any point $(x, u)$ of its tangent bundle $T M$, the tangent space of $T M$ splits into the horizontal and vertical subspaces:

$$
(T M)_{(x, u)}=H_{(x, u)} \oplus V_{(x, u)} .
$$

Each tangent vector $\tilde{Z} \in(T M)_{(x, u)}$ can be written in the form $\tilde{Z}=X^{h}+Y^{v}$, where $X, Y \in M_{x}$ are uniquely determined vectors.

The tangent bundle $T M$ can be endowed in a natural way with a semi-Riemannian metric, the Sasaki metric $G$, depending only on the semi-Riemannian metric $g$. It is determined by

$$
G_{z}\left(X^{h}, Y^{h}\right)=G_{z}\left(X^{v}, Y^{v}\right)=g_{x}(X, Y), \quad G_{z}\left(X^{h}, Y^{v}\right)=0,
$$

for any $z=(x, u) \in T M$ and for any $X, Y \in M_{x}$. G is a semi-Riemannian metric of signature $(2 v, 2 n-2 v)$, and both $H_{z}$ and $V_{z}$ have index $v$. There is also an almost complex structure $J$ on $T M$ given by

$$
J X^{h}=X^{v}, \quad J X^{v}=-X^{h},
$$

then the Sasaki metric $G$ is Hermitian with respect to the almost complex structure $J$. We denote by $\mathcal{N}_{(x, u)}$ the canonical vertical vector field on TM and by $\zeta_{(x, u)}$ the geodesic flow on TM. They are defined by

$$
\mathcal{N}_{(x, u)}=u_{(x, u)}^{v} \quad \text { and } \quad \zeta_{(x, u)}=u_{(x, u)}^{h}
$$


The Liouville form $\beta$ on $T M$, defined by

$$
\beta(\tilde{X})_{z}=G\left(\tilde{X}_{z}, \zeta_{z}\right)=g_{x}\left(\pi_{* z} \tilde{X}_{z}, u\right),
$$

satisfies the following (see Prop. 2 of [84], and [2] p. 139)

$$
2(\mathrm{~d} \beta)(\tilde{X}, \tilde{Y})=G(\tilde{X}, J \tilde{Y}),
$$

that is, $2(\mathrm{~d} \beta)$ is the fundamental 2-form, and so $(T M, J, G)$ is an indefinite almost Kaehler manifold. Besides (see ([84], Proposition 3): $J$ is integrable if and only if the semi-Riemannian manifold $(M, g)$ is locally isometric to the semi-Euclidean space $\mathbb{R}_{v}^{n}$.

Consider the tangent hyperquadric bundle

$$
T_{\mathcal{E}}(M, g)=\left\{(x, u) \in T M \mid g_{x}(u, u)=\varepsilon\right\}, \quad \varepsilon \in\{ \pm 1\} .
$$

The vertical vector field $\mathcal{N}$ is normal to $T_{\mathcal{\varepsilon}}(M, g)$ in $(T M, G)$ and $G(\mathcal{N}, \mathcal{N})=\varepsilon$ along $T_{\mathcal{\varepsilon}}(M, g)$, moreover the geodesic flow $\zeta$ is tangent to $T_{\varepsilon}(M, g)$. Any horizontal vector $X^{h}$ is tangent to $T_{\varepsilon}(M, g)$, and a vertical vector $X^{v}$ is tangent to $T_{\varepsilon}(M, g)$ if and only if $X^{v}$ is orthogonal to $\mathcal{N}_{z}$. Consequently, the tangent space of $T_{\mathcal{\varepsilon}}(M, g)$, at a point $z=(x, u) \in T_{\mathcal{\varepsilon}}(M, g)$, is given by

$$
\left(T_{\mathcal{\varepsilon}}(M, g)\right)_{z}=\left\{X^{h}+Y^{v} / X \in M_{x}, Y \in\{u\}^{\perp} \subset M_{x}\right\} .
$$

In general, the tangential lift of a vector field $X$ is a vector field on $T_{\varepsilon}(M, g)$ defined by

$$
X^{t}=X^{v}-\varepsilon G\left(X^{v}, \mathcal{N}\right) \mathcal{N} \text {, that is, } X_{z}^{t}=X_{z}^{v}-\varepsilon g_{x}(X, u) u_{z}^{v} .
$$

The Sasaki metric on $T_{\mathcal{\varepsilon}}(M, g)$ is the semi-Riemannian metric $\widetilde{G}$ on $T_{\mathcal{\varepsilon}}(M, g)$ induced from $G$, it is completely determined by the identities

$$
\left\{\begin{array}{l}
\widetilde{G}_{z}\left(X^{h}, Y^{h}\right)=g_{x}(X, Y), \\
\widetilde{G}_{z}\left(X^{h}, Y^{t}\right)=0 \\
\widetilde{G}_{z}\left(X^{t}, Y^{t}\right)=g_{x}(X, Y)-\varepsilon g_{x}(X, u) g_{x}(Y, u),
\end{array}\right.
$$

for all $z=(x, u) \in T_{\varepsilon}(M, g)$ and $X, Y \in M_{x}$. Since the Sasaki metric on the tangent bundle TM is of signature $(2 v, 2 n-2 v), G(\mathcal{N}, \mathcal{N})=\varepsilon$ and $T_{\varepsilon}(M, g)$ is an orientable semi-Riemannian hypersurface of $(T M, G)$ of $\operatorname{sign} \varepsilon$, then

$$
\text { the index of } T_{-1}(M, g) \text { is } 2 v-1 \text { and the index of } T_{1}(M, g) \text { is } 2 v \text {. }
$$

We now construct the standard non-degenerate almost CR structure on $T_{\varepsilon}(M, g)$. The tangent hyperquadric bundle $T_{\varepsilon}(M, g)$ is an orientable non-degenerate hypersurface of the indefinite almost Kaehler manifold $(T M, J, G)$. Then, by the usual procedure, we construct the almost contact semi-Riemannian structure $\left(\tilde{\xi}^{\prime}, \eta^{\prime}, \varphi^{\prime}, \tilde{G}\right)$ induced on $T_{\varepsilon}(M, g)$, where

$$
\begin{aligned}
\eta_{z}^{\prime}\left(\tilde{X}_{z}\right) & =\varepsilon \tilde{G}\left(\tilde{X}_{z}, \xi_{z}^{\prime}\right)=\varepsilon G\left(\tilde{X}_{z}, u_{z}^{h}\right)=\varepsilon \beta_{z}\left(\tilde{X}_{z}\right) \quad \text { (the Liouville form) } \\
\xi_{z}^{\prime} & =-J \mathcal{N}_{z}=u_{z}^{h}=\zeta_{z} \quad \text { (the geodesic flow) } \\
\varphi^{\prime}\left(\tilde{X}_{z}\right) & =J \tilde{X}_{z}-\varepsilon G\left(J \tilde{X}_{z}, \mathcal{N}_{z}\right) \mathcal{N}_{z} \quad \text { (the tangential component) }
\end{aligned}
$$

for $z=(x, u) \in T_{\varepsilon}(M, g)$ and $\tilde{X}$ vector field on $T_{\varepsilon}(M, g)$. Since $2 \varepsilon\left(\mathrm{d} \eta^{\prime}\right)(\tilde{X}, \tilde{Y})=\tilde{G}\left(\tilde{X}, \varphi^{\prime} \tilde{Y}\right)$ for any $\tilde{X}, \tilde{Y}$ vector fields on $T_{\varepsilon}(M, g)$, if we rescale the structure tensors appropriately by

$$
\eta=(1 / 2 \varepsilon) \eta^{\prime}, \quad \xi=2 \varepsilon \tilde{\zeta}^{\prime}, \quad \varphi=\varphi^{\prime} \quad \text { and } \quad \bar{g}=(1 / 4) \tilde{G},
$$


we get the standard contact semi-Riemannian structure on $T_{\mathcal{E}} M$. In explicite form these tensors are given by

$$
\begin{gathered}
\eta\left(X_{z}^{t}\right)=0, \quad \eta\left(X_{z}^{h}\right)=\frac{1}{2} g\left(X_{x}, u\right), \quad \xi_{z}=2 \varepsilon u_{z}^{h} \\
\varphi\left(X_{z}^{t}\right)=-X_{z}^{h}+\frac{1}{2} g\left(X_{x}, u\right) \bar{\xi}_{z}, \quad \varphi\left(X_{z}^{h}\right)=X_{z}^{t}, \\
\left\{\begin{array}{c}
\bar{g}\left(X_{z}^{h}, Y_{z}^{h}\right)=\frac{1}{4} g_{x}\left(X_{x}, Y_{x}\right), \\
\bar{g}\left(X_{z}^{h}, Y_{z}^{t}\right)=0, \\
\bar{g}\left(X_{z}^{t}, Y_{z}^{t}\right)=\frac{1}{4}\left(g_{x}\left(X_{x}, Y_{x}\right)-\varepsilon g_{x}\left(X_{x}, u\right) g_{x}\left(Y_{x}, u\right)\right),
\end{array}\right.
\end{gathered}
$$

for any $X, Y$ vector fields on $M$ and $z=(x, u) \in T_{\mathcal{E}}(M, g)$, in particular $\bar{g}(\xi, \xi)=\varepsilon$. Hence, the corresponding standard non-degenerate almost $\mathbf{C R}$ structure $(\mathcal{H}, J, \theta)$ on $T_{\mathcal{E}}(M, g)$ is defined by

$$
\mathcal{H}=\operatorname{ker} \eta, \quad \theta=-\eta, \quad J=\varphi_{\mid \operatorname{ker} \eta} .
$$

Besides, the corresponding Levi form $L_{\theta}$ is defined by

$$
L_{\theta}\left(X_{z}^{h}, Y_{z}^{t}\right)=0, \quad L_{\theta}\left(X_{z}^{h}, Y_{z}^{h}\right)=L_{\theta}\left(X_{z}^{t}, Y_{z}^{t}\right)=(1 / 4) g_{x}\left(X_{x}, Y_{x}\right)
$$

for any $z=(x, u) \in T_{\mathcal{E}}(M, g), X, Y \in M_{x}, X, Y \perp u$.

A natural problem for the standard non-degenerate almost CR structure $(\mathcal{H}, J, \theta)$ on $T_{\mathcal{E}}(M, g)$ is to see when it is a CR structure. By using

- formulas which give the Levi-Civita connection $\tilde{\nabla}$ of $\tilde{G}$ in terms of $\nabla$ and $R$ (the Levi-Civita connection and the curvature tensor of $g$ );

- $\quad$ he generalized Tanaka-Webster connection $\hat{\nabla}$ associated to the standard non-degenerate almost CR structure $(\mathcal{H}, J, \theta)$, and

- $\quad$ a result of M.Dajczer - K.Nomizu [86] on the sectional curvatures of indefinite metrics, we get

Theorem 25. Let $(M, g)$ be a semi-Riemannian manifold with $\operatorname{dim} M=n \geq 3$. Then, the standard non-degenerate almost $C R$ structure $(\mathcal{H}, J, \theta)$ on $T_{\mathcal{E}}(M, g)$ is integrable, i.e., it is a non-degenerate CR structure if, and only if, $(M, g)$ has constant sectional curvature.

It is interesting to note that if $(M, g)$ is a semi-Riemannian manifold of constant sectional curvature $c \neq 0$, and of dimension $n \geq 2$, then $(T M, J)$ is not integrable but, by Theorem 25 , the standard non-degenerate almost CR structure $(\mathcal{H}, J, \theta)$ on $T_{\mathcal{E}}(M, g)$ is integrable.

As a consequence of Theorems 18 and 25, we get

Corollary 6. Let $\left(M, \mathcal{H}_{0}, J_{0}, \theta_{0}\right)$ be a non-degenerate $C R$ manifold, $\operatorname{dim} M=2 n+1 \geq 5$. Then, the standard non-degenerate almost $C R$ structure $(\mathcal{H}, J, \theta)$ of $T_{\mathcal{E}}(M, g)$ is integrable, i.e., is a non-degenerate $C R$ structure, if and only if the Webster metric $g_{\theta_{0}}$ of $M$ has constant sectional curvature $c=\varepsilon$ and the pseudohermitian torsion $\tau_{0}$ vanishes.

Remark 13. If we set $T_{0}(M, g)_{x}=\left\{u \in T_{x}(M) \backslash\left\{0_{x}\right\} \mid g_{x}(u, u)=0\right\}, \quad x \in M$, then $T_{0}(M, g) \rightarrow M$ is a bundle of nullcones, and in such a case we can not consider the standard contact semi-Riemannian structure on $T_{0}(M, g)$. In fact, also in this case the geodesic flow $\zeta_{(x, u)}$, for $(x, u) \in T_{0}(M, g)$, is tangent to $T_{0}(M, g)$, but now it is a lightlike vector field while the Reeb vector field is never lightlike.

In the Riemannian case Y. Tashiro (see [2], Section 9.2) proved that the standard contact Riemannian structure on $T_{1} M$ is $K$-contact, equivalently the geodesic flow is Killing, if and only if the 
Riemannian manifold $(M, g)$ has constant sectional curvature +1 , and in such case the standard contact Riemannian structure on $T_{1} M$ is Sasakian. Now, we consider the same question in the semi-Riemannian setting and in terms of CR geometry.

By using

- formulas for the pseudohermitian torsion of $T_{\mathcal{E}}(M, g)$ :

$$
\tau X_{z}^{h}=-\varepsilon X_{z}^{t}+\varepsilon(R(X, u) u)_{z}^{t}, \quad \tau X_{z}^{t}=-\varepsilon X_{z}^{h}+\frac{\varepsilon}{2} g_{x}(X, u) \bar{\xi}_{z}+\varepsilon(R(X, u) u)_{z}^{h}
$$

- a result of K. Nomizu [87] on the sectional curvatures of indefinite metrics;

- Theorems 14 and 25, Equations (48) and (49);

we get the following

Theorem 26. Let $(M, g)$ be a semi-Riemannian manifold of index $v, 0 \leq v \leq n=\operatorname{dim} M$. Then, we have the following

(i) The standard non-degenerate almost $C R$ structure $(\mathcal{H}, \theta, J)$ on $T_{\mathcal{E}}(M, g)$ has vanishing pseudohermitian torsion if and only if $(M, g)$ has constant sectional curvature $c=\varepsilon$.

In such a case $(\mathcal{H}, \theta, J)$ is a pseudo-Einstein CR structure, which is Sasakian, and the Ricci tensor and the pseudohermitian Ricci tensor are given by

$$
\bar{R} i c=2 \varepsilon(2 n-3) \bar{g}-2(n-2) \eta \otimes \eta, \quad \hat{R} i c=4 \varepsilon(n-1) L_{\theta}
$$

(ii) If $0<v<n$, the pseudo-Einstein CR structure of (i) is Einstein, i.e., the Webster metric is Einstein, if and only if $(M, g)$ is a Lorentzian surface of constant curvature $c=\varepsilon$. In such a case, $T_{\varepsilon}(M, g)$ has constant sectional curvature $c=\varepsilon$.

Corollary 7. Let $(M, g)$ be a semi-Riemannian manifold of index $v, 0 \leq v \leq n=\operatorname{dim} M$. Then, the geodesic flow of $T_{\mathcal{\varepsilon}}(M, g)$ is Killing if and only if $M$ has constant sectional curvature $\varepsilon$.

\subsection{Sasaki-Einstein and H-Contact Structures on $T_{\mathcal{E}} M$}

The geometry of a $H$-contact unit tangent sphere bundles when the base manifold is a Riemannian manifold has been extensively investigated (see, for example, Refs. [88-92]).

In the semi-Riemannian case, we have

Theorem 27. Let $(M, g)$ be a semi-Riemannian manifold of constant sectional curvature $c$. Then, the standard contact semi-Riemannian structure $(\eta, \xi, \varphi, \bar{g})$ on $T_{\varepsilon}(M, g)$ is $H$-contact. Moreover, the structure is $\eta$-Einstein if and only if either $c=\varepsilon$ or $c=(n-2) \varepsilon, n=\operatorname{dim} M$. In such a case, the Ricci tensor is given by

$$
\bar{R} i c=2 \varepsilon\left(2(n-2)+c^{2}\right) g-2(n-2) c^{2} \eta \otimes \eta
$$

where $c=\varepsilon$ or $c=(n-2) \varepsilon$.

Remark 14. Recall that $\eta$-Einstein, K-contact and Sasakian semi-Riemannian manifolds are H-contact. Now, we remark that:

- $\quad$ for $c \neq \varepsilon$, Theorem 27 gives examples of H-contact semi-Riemannian manifolds which are not K-contact;

- $\quad$ for $c \neq \varepsilon$ and $c \neq(n-2) \varepsilon$, Theorem 27 gives examples of H-contact semi-Riemannian manifolds which are not $\eta$-Einstein.

As a consequence of Theorems 25-27, we obtain the following result. 
Theorem 28. Let $(M, g)$ be a semi-Riemannian manifold, $\operatorname{dim} M=n \geq 3$.

(i) If $n>3$, then the standard non-degenerate almost $C R$ structure on $T_{\mathcal{E}}(M, g)$ is an $\eta$-Einstein $C R$ structure if and only if $(M, g)$ has constant sectional curvature $c=\varepsilon$ or $c=(n-2) \varepsilon \neq \varepsilon$.

(j) If $n=3$, then the standard non-degenerate almost $C R$ structure on $T_{\mathcal{E}}(M, g)$ is an $\eta$-Einstein $C R$ structure if and only if $(M, g)$ has constant sectional curvature $c=\varepsilon$. Moreover, in such case the structure is pseudo-Einstein and Sasakian.

In general, for a contact semi-Riemannian manifold, the Reeb vector field $\xi$ infinitesimal harmonic transformation does not imply $K$-contact (cf. Example 3). If $(M, g)$ is a semi-Riemannian $n$-manifold of constant sectional curvature $c$, by Theorem 27 the standard contact semi-Riemannian structure $(\xi, \eta, \varphi, \bar{g})$ on $T_{\mathcal{E}}(M, g)$ is $H$-contact. Moreover, by using Equation (66), we get

$$
\operatorname{tr} h^{2}=\operatorname{tr} \tau^{2}=2 n(c-\varepsilon)^{2} .
$$

Then, by using Equation (27), we get

Corollary 8. Let $(M, g)$ be a semi-Riemannian manifold of constant sectional curvature c. Then, the Reeb vector field of the standard contact semi-Riemannian structure on $T_{\mathcal{E}}(M, g)$ is an infinitesimal harmonic transformation if and only if the pseudohermitian torsion vanishes (i.e., the structure is K-contact and $c=\varepsilon$ ).

Now, given a $K$-contact semi-Riemannian structure $(\eta, \xi, \varphi, g)$ on $M$, $\operatorname{dim} M=2 n+1>3$, consider the new K-contact semi-Riemannian structure $(\tilde{\eta}, \tilde{\xi}, \tilde{\varphi}, \tilde{g})$ defined by the $\mathcal{D}$-homothetic deformation (or transverse homothety) Equation (16). Then, by Equations (18) and (19), the Ricci tensors and the scalar curvatures of $g$ and $\tilde{g}(\tilde{\varepsilon}=\varepsilon)$ are related by

$$
\tilde{R} i c=R i c-2 \varepsilon(t-1) g+2(t-1)(n t+n+1) \eta \otimes \eta, \quad \tilde{r}=\frac{r+2 n \varepsilon}{t}-2 n \varepsilon .
$$

In particular, if $(\eta, g)$ is $\eta$-Einstein, that is,

$$
\text { Ric }=\left(\frac{r}{2 n}-\varepsilon\right) g+\left(-\frac{r \varepsilon}{2 n}+2 n+1\right) \eta \otimes \eta
$$

where the scalar curvature $r$ is a constant because $n>1$, then the Ricci tensor of the new $K$-contact semi-Riemannian structure $(\tilde{\eta}, \tilde{g})$ is given by

$$
\begin{aligned}
\tilde{R} i c & =\left(\frac{r+2 n \varepsilon}{2 n t}-2 \varepsilon\right) \tilde{g}-\left(\frac{r \varepsilon+2 n}{2 n t}-2(n+1)\right) \tilde{\eta} \otimes \tilde{\eta} \\
& =\left(\frac{\tilde{r}}{2 n}-\varepsilon\right) \tilde{g}-\left(\frac{\tilde{r} \varepsilon}{2 n}-(2 n+1)\right) \tilde{\eta} \otimes \tilde{\eta} \\
& =\left(\frac{\hat{r}}{2 n t}-2 \varepsilon\right) \tilde{g}-\left(\frac{\varepsilon \hat{r}}{2 n t}-2(n+1)\right) \tilde{\eta} \otimes \tilde{\eta},
\end{aligned}
$$

where $\hat{r}=r+2 n \varepsilon$ is the Tanaka-Webster scalar curvature (see Equation (49)) of the $\eta$-Einstein $K$-contact semi-Riemannian structure $(\eta, g)$. So, for any $t \neq 0$, the new $K$-contact semi-Riemannian structure $(\tilde{\eta}, \tilde{g})$ is also $\tilde{\eta}$-Einstein. Moreover, if the scalar curvature $r$ of the $\eta$-Einstein $K$-contact semi-Riemannian manifold $(\eta, g)$ satisfies $r \neq-2 n \varepsilon$, that is, the Tanaka-Webster scalar curvature $\hat{r} \neq 0$, then for $t=\frac{\varepsilon \hat{r}}{4 n(n+1)} \neq 0$, the corresponding $K$-contact semi-Riemannian structure $(\tilde{\eta}, \tilde{g})$ is Einstein. Then, we get

Proposition 17. Let $(M, \eta, g)$ be an $\eta$-Einstein K-contact semi-Riemannian $(2 n+1)$-manifold, $n>1$. If the Tanaka-Webster scalar curvature satisfies $\hat{r} \neq 0$, then there exists a unique transverse homothety whose resulting structure $(\tilde{\eta}, \tilde{g})$ is an Einstein K-contact semi-Riemannian structure. 
Remark 15. If the scalar curvature $r$ of the $\eta$-Einstein $K$-contact semi-Riemannian manifold $(\eta, g)$ satisfies $r=-2 n \varepsilon$, equivalently $\hat{r}=0$, then by using Equations (48) and (49), the corresponding non-degenerate almost $C R$ structure is pseudo-Ricci flat, that is, the pseudohermitian Ricci tensor R̂ic vanishes.

Now, we apply the above considerations to the tangent hyperquadric bundle $T_{\mathcal{E}}(M, g)$, where $(M, g)$ is a semi-Riemannian manifold of constant sectional curvature $c, \operatorname{dim} M=n \geq 2$.

If $c=\varepsilon$, from Theorem 26 we know that $\left(T_{\mathcal{\varepsilon}}(M, g), \eta, \bar{g}\right)$ is Einstein if and only if $n=2$, i.e., $(M, g)$ is a surface of constant curvature $\varepsilon$. In such a case, $\left(T_{\varepsilon}(M, g), \bar{g}\right)$ is of constant sectional curvature $\varepsilon$.

If $c \neq \varepsilon$ and $0<v<n$, by using Equation (68), $\left(T_{\varepsilon}(M, g), \eta, \bar{g}\right)$ is Einstein if and only if $(M, g)$ is a flat Lorentzian surface. In such a case, $\left(T_{\varepsilon}(M, g), \bar{g}\right)$ is a flat contact Lorentzian 3-manifold.

In general, if $c=\varepsilon$, Theorem 26 gives that $\left(T_{\varepsilon}(M, g), \eta, \bar{g}\right)$ is is $\eta$-Einstein Sasakian with constant scalar curvature

$$
\bar{r}=2(n-1)(4 n-5) \varepsilon=2 m(4 m-1) \varepsilon \neq-2 m \varepsilon,
$$

where $\operatorname{dim} T_{1} M=2 m+1 \geq 3, m=n-1$. Then, by the $\mathcal{D}$-homothetic deformation Equation (16) with $t=\varepsilon \hat{r} / 4 m(m+1)=2(n-1) / n$, we get (cf, also Proposition 17) the following

Theorem 29. Let $(M, g)$ be a semi-Riemannian manifold, $\operatorname{dim} M=n \geq 2$, of constant sectional curvature $\varepsilon$. Then, the tangent hyperquadric bundle $T_{\mathcal{E}}(M, g)$ admits a Sasaki-Einstein structure $(\tilde{\eta}, \tilde{\xi}, \tilde{\varphi}, \tilde{g})$ given by:

$$
\begin{aligned}
& \tilde{\eta}=(2(n-1) / n) \eta, \quad \tilde{\xi}=(n / 2(n-1)) \xi, \quad \tilde{\varphi}=\varphi, \quad \text { and } \\
& \tilde{g}=(2(n-1) / n) \bar{g}-\frac{2(n-1)(n-2)}{n^{2}} \eta \otimes \eta,
\end{aligned}
$$

where $(\eta, \bar{\zeta}, \varphi, \bar{g})$ is the standard contact semi-Riemannian structure.

Remark 16. We note that for $n=2$ the two structures $(\eta, \xi, \varphi, \bar{g})$ and $(\tilde{\eta}, \tilde{\xi}, \tilde{\varphi}, \tilde{g})$ coincide, on the other hand for $n=2$ the structure $(\xi, \eta, \varphi, g)$ is Einstein.

Example 6. Consider the pseudo-sphere $S_{2 s}^{2 n+1}(1)$ and the pseudo-hyperbolic space $H_{2 s-1}^{2 n+1}(-1)$. Then, the tangent pseudo-sphere bundle $T_{1}\left(S_{2 s}^{2 n+1}(1)\right)$ and the tangent pseudo-hyperbolic bundle $T_{-1}\left(H_{2 s-1}^{2 n+1}(-1)\right)$ admit a Sasaki-Einstein structure.

If we assume that $(M, g)$ is Lorentzian $(v=1)$, then $\left(T_{-1}(M, g), \tilde{G}\right)$ is Lorentzian. Then, from Theorem 26, we get

Theorem 30. Let $(M, g)$ be a n-dimensional Lorentzian manifold. Then the standard nondegenerate almost $C R$ structure on $T_{-1}(M, g)$ has vanishing pseudohermitian torsion if and only if $(M, g)$ has constant sectional curvature $c=-1$. Moreover, in such a case the corresponding standard contact Lorentzian structure $(\eta, \xi, \varphi, \bar{g})$ is Sasakian $\eta$-Einstein with scalar curvature

$$
\bar{r}=-2(n-1)(4 n-5) .
$$

As an application in relativity theory, we have the the following

Corollary 9. Let $(M, g)$ be a complete simply connected 4-dimensional Lorentzian manifold. Then, the standard nondegenerate almost $C R$ structure on $T_{-1}(M, g)$ has vanishing pseudohermitian torsion if and only if $(M, g)$ is isometric to the universal anti-de Sitter space $\tilde{H}_{1}^{4}(-1)$.

Remark 17. The standard examples of non Sasakian $(\kappa, \mu)$-spaces are the tangent sphere bundles of Riemannian space forms of constant curvature c different from -1 (see, for instance, Ref. [2] Teorem 7.9). In such a case, the corresponding Boeckx invariant defined by Equation (55) is given by: 


$$
I_{T_{1} M}=\frac{1+c}{|1-c|}
$$

Therefore as $c$ varies over the reals, $I_{T_{1} M}$ assumes all the real values strictly greater than $>-1$. Boeckx found examples of $(\kappa, \mu)$-spaces, for every value of the invariant $I \leq-1$, namely a two parameter family of Lie groups with a left-invariant contact metric structure (cf. [62], and [2] pp. 125-126).

More recently, E. Loiudice and A. Lotta [93] showed that the tangent hyperquadric bundles $T_{-1} M$ over Lorentzian space forms $(M, g)$ of constant curvature $c$ different from -1 , equipped with a strictly pseudoconvex $C R$ structure, also provide non equivalent examples. For these space, the formula for the Boeckx invariant changes as follows:

$$
I_{T_{-1} M}=\frac{c-1}{|c+1|}
$$

where $c \in \mathbb{R}, c \neq-1$, so that for $c \leq 0$, these examples cover all possible values of the Boeckx invariant in $(-\infty,-1)$. This result makes E. Boeckx's classification of $(\kappa, \mu)$-spaces in [62] more geometric. We note that in this case the Webster metric of $T_{-1} M$ is not the Lorentzian metric Equation (65) induced from the Sasaki metric of TM.

\section{Levi Harmonicity on Non-Degenerate Almost CR Manifolds}

The papers $[47,94]$ are devoted to the study of a class of variational principles whose corresponding Euler-Lagrange equations are degenerate elliptic and generalize ordinary harmonic map theory in the spirit of sub-Riemannian geometry (cf. [95]) i.e., given a smooth map $f: M \rightarrow M^{\prime}$ of (semi) Riemannian manifolds $(M, g)$ and $\left(M^{\prime}, g^{\prime}\right)$ one replaces the Hilbert-Schmidt norm of $d f$ by the trace with respect to $g$ of the restriction of $f^{*} g^{\prime}$ to a given codimension one distribution $\mathcal{H}$ on $M$ (rather than applying the same construction to the full $f^{*} g^{\prime}$ ). E. Barletta et al., Ref. [96], introduced pseudoharmonic maps $f: M \rightarrow M^{\prime}$ from a nondegenerate CR manifold $M$ endowed with a contact form $\theta$ into a Riemannian manifold $M^{\prime}$. When $M^{\prime}$ is itself a non-degenerate CR manifold carrying the contact form $\theta^{\prime}$ a result in [96] describes pseudoharmonicity of CR maps $f: M \rightarrow M^{\prime}$. R. Petit [97] considered the following (pseudohermitian analog to the) second fundamental form

$$
\beta_{f}(X, Y)=\widetilde{\nabla}_{X}^{\prime} f_{*} Y-f_{*} \hat{\nabla}_{X} Y, \quad X, Y \in \mathfrak{X}(M),
$$

where $\hat{\nabla}$ is the Tanaka-Webster connection of $M$ and $\widetilde{\nabla}^{\prime}=f^{-1} \nabla^{\prime}$ is the pullback of the Levi-Civita connection $\nabla^{\prime}$ of $M^{\prime}$. The approach in [96] is to replace $\nabla^{\prime}$ by an arbitrary linear connection $D^{\prime}$ on $M^{\prime}$, consider the restriction $\Pi_{\mathcal{H}} \beta_{f}$ of (69) to the Levi distribution $\mathcal{H}=\operatorname{ker} \theta$, and take the trace with respect to the Levi form $L_{\theta}$. Then $f$ is called pseudoharmonic (with respect to the data $\left(\theta, D^{\prime}\right)$ ) if $\operatorname{trace}_{L_{\theta}}\left(\Pi_{\mathcal{H}} \beta_{f}\right)=0$.

More recently, Dragomir and R. Petit et al., [98], studied contact harmonic maps, i.e., $C^{\infty}$ maps $f: M \rightarrow M^{\prime}$ from a compact strictly pseudoconvex $C R$ manifold $M$ into a contact Riemannian manifold $M^{\prime}$ which are critical points of the functional

$$
E(f)=\frac{1}{2} \int_{M}\left\|(d f)_{\mathcal{H}, \mathcal{H}^{\prime}}\right\|^{2} \theta \wedge(d \theta)^{n},
$$

where $\theta$ is a contact form on $M$ and $(d f)_{\mathcal{H}, \mathcal{H}^{\prime}}=\operatorname{pr}_{\mathcal{H}^{\prime}} \circ f_{*}: \mathcal{H} \rightarrow \mathcal{H}^{\prime}$.

J. Konderak \& R. Wolak, Ref. [99], introduced transversally harmonic maps as foliated maps $f:(M, \mathcal{F}, g) \rightarrow\left(M^{\prime}, \mathcal{F}^{\prime}, g^{\prime}\right)$ between foliated Riemannian manifolds satisfying a condition similar to the vanishing of the tension field in Riemannian geometry.

As a natural continuation of the ideas in [96], and following the ideas of B. Fuglede (who started the study of the semi-Riemannian case within harmonic map theory, cf. [100], and [101] pp. 427-455), in the papers [47,94], S. Dragomir and the present author introduced the concept of Levi harmonic map $f$ from an almost contact semi-Riemannian manifold $(M, \eta, \xi, \varphi, g)$ into a semi-Riemannian manifold $\left(M^{\prime}, g^{\prime}\right)$, i.e., $C^{\infty}$ solutions of $\tau_{\mathcal{H}}(f) \equiv \operatorname{trace}_{g}\left(\Pi_{\mathcal{H}} \beta_{f}\right)=0$, where $\beta_{f}$ is the second fundamental form 
of $f$, and $\Pi_{\mathcal{H}} \beta_{f}$ is the restriction of $\beta_{f}$ to the Levi distribution $\mathcal{H}=$ ker $\eta$. Thus, we studied the Levi harmonicity for $\mathrm{CR}$ maps between two almost contact semi-Riemannian manifolds. This is perhaps the most general geometric setting (metrics are semi-Riemannian, in general the contact condition is not satisfied and the underlying almost CR structures are not integrable). In such a study, an important role is played by the notion of $\varphi$-condition:

$$
\nabla_{\varphi X} \varphi X+\nabla_{X} X=\varphi[\varphi X, X], \quad \text { equivalently }:\left(\nabla_{X} \varphi\right) \varphi X=\left(\nabla_{\varphi X} \varphi\right) X,
$$

for any $X \in \mathcal{H}$. Moreover, as emphasized in [47], the class of almost contact semi-Riemannian manifolds obeying to Equation (70) is quite large. For instance, contact semi-Riemannian manifolds, orientable real hypersurfaces in an indefinite Kaehler manifold (with the induced almost contact semi-Riemannian structure) and quasi-cosimplectic manifolds (which contains cosymplectic and almost cosympletic manifolds) satisfy the $\varphi$-condition. Moreover, the $\varphi$-condition extends (cf. [94], Section 3) the so-called condition $(A)$ of Rawnsley [102]. Rawnsley in his paper introduced the condition $(A)$ in order to study the harmonicity of $f$-holomorphic maps between an almost Hermitian manifold with coclosed Kaehler form and a Riemannian manifold equipped with a $f$-structure. Moreover, there is the following characterization of a contact Riemannian manifold ([103], Theorem 3.2): an almost contact Riemannian manifold is a contact Riemannian manifold if and only the following conditions are satisfied: the tensor $h=(1 / 2) \mathcal{L}_{\xi} \varphi$ is symmetric, and

$$
\left(\nabla_{X} \varphi\right) Y+\left(\nabla_{\varphi X} \varphi\right) \varphi Y=2 g(X, Y) \xi-\eta(Y) X-\eta(X) \eta(Y) \xi-\eta(Y) h X \text { for any } X, Y \in \mathfrak{X}(M) .
$$

This last condition, for $Y=\varphi X, X \in \operatorname{ker} \eta$, implies the $\varphi$-condition.

In this Section we report some results of $[47,94]$, for almost contact semi-Riemannian manifolds. Let $(M, \eta, \xi, \varphi, g)$ be a real $(2 n+1)$-dimensional almost contact semi-Riemannian manifold and $\left(M^{\prime}, g^{\prime}\right)$ a semi-Riemannian manifold. Let $f: M \rightarrow M^{\prime}$ be a $C^{\infty}$ map and $f^{-1} T\left(M^{\prime}\right) \rightarrow M$ the pullback of $T\left(M^{\prime}\right)$ by $f$. Let $\widetilde{\nabla}^{\prime}=f^{-1} \nabla^{\prime}$ be the pullback of the Levi-Civita connection $\nabla^{\prime}$ of $\left(M^{\prime}, g^{\prime}\right)$ i.e., the connection in the vector bundle $f^{-1} T\left(M^{\prime}\right) \rightarrow M$ induced by $\nabla^{\prime}$. If $\left(U, x_{i}\right)$ and $\left(U^{\prime}, y_{\alpha}\right)$ are local coordinate systems on $M$ and $N$ such that $f(U) \subset V$ then $\tilde{\nabla}^{\prime}$ is locally described by

$$
\widetilde{\nabla}_{\partial / \partial x_{j}}^{\prime}\left(\partial / \partial y_{\beta}\right)^{f}=\frac{\partial f^{\alpha}}{\partial x_{j}}\left(\Gamma_{\alpha \beta}^{\prime \gamma} \circ f\right)\left(\partial / \partial y_{\gamma}\right)^{f}
$$

where $Y^{f}=Y \circ f \in C^{\infty}\left(f^{-1}\left(U^{\prime}\right), f^{-1} T\left(M^{\prime}\right)\right)$ denotes the natural lift of $Y \in \mathfrak{X}\left(U^{\prime}\right)$ and $\Gamma_{\alpha \beta}^{\prime \gamma}$ are the Christoffel symbolds of $\left(M^{\prime}, g^{\prime}\right)$. Let $\mathcal{H}=$ ker $\eta$ and $J=\left.\varphi\right|_{\mathcal{H}}$ be the almost CR structure underlying $(\eta, \xi, \varphi, g)$. The second fundamental form $\beta_{f}$ of $f$ is given by

$$
\beta_{f}(X, Y)=\widetilde{\nabla}_{X}^{\prime} f_{*} Y-f_{*} \nabla_{X} Y, \quad X, Y \in \mathfrak{X}(M)
$$

Here $\nabla$ is the Levi-Civita connection of $(M, g)$ and the vector field $f_{*} X$ is given by $\left(f_{*} X\right)(x)=\left(f_{* x}\right) X_{x} \in T_{f(x)} M^{\prime}$ for any $x \in M$ and $X \in \mathfrak{X}(M)$. Next, let $\tau_{\mathcal{H}}(f) \in C^{\infty}\left(f^{-1} T M^{\prime}\right)$ be the tension field defined by

$$
\tau_{\mathcal{H}}(f)=\operatorname{trace}_{g}\left(\Pi_{\mathcal{H}} \beta_{f}\right)
$$

where $\Pi_{\mathcal{H}} \beta_{f}$ is the restriction of $\beta_{f}$ to $\mathcal{H} \otimes \mathcal{H}$. Note that the tension field $\tau(f)=\tau_{\mathcal{H}}(f)+\varepsilon\left(\tilde{\nabla}_{\xi}^{\prime} f_{*} \xi-f_{*} \nabla_{\xi} \xi\right), \varepsilon=g(\xi, \xi)$.

Definition 6. Let $(M, \eta, \xi, \varphi, g)$ an almost contact semi-Riemannian manifold and $\left(M^{\prime}, g^{\prime}\right)$ a semi-Riemannian manifold. A $C^{\infty}$ map $f: M \rightarrow M^{\prime}$ is said to be Levi harmonic with respect to $\mathcal{H}=$ ker $\eta$ if $\tau_{\mathcal{H}}(f)=0$. 
In the case of a CR map between two almost contact semi-Riemannian manifolds, we have the following

Theorem 31. ([94]) Let $(M, \eta, \xi, \varphi, g)$ and $\left(M^{\prime}, \eta^{\prime}, \xi^{\prime}, \varphi^{\prime}, g^{\prime}\right)$ be two almost contact semi-Riemannian manifolds with $\operatorname{dim}(M)=2 n+1$. Then, for each $C R$ map $f: M \rightarrow M^{\prime}$ we have

$$
\begin{aligned}
\tau_{\mathcal{H}}(f)= & -\operatorname{trace}(\varphi \nabla \xi) \tilde{\varphi}^{\prime} f_{*} \xi+\tilde{\varphi}^{\prime}\left(\operatorname{trace}_{\mid \mathcal{H}} f^{*} \nabla^{\prime} \varphi^{\prime}\right)-\left(\operatorname{trace}_{\mid \mathcal{H}} f^{*} \nabla^{\prime} \eta^{\prime}\right) \xi^{\prime} \\
& +f_{*}\left(\varphi \nabla^{*} \varphi+(\operatorname{div} \xi) \xi+\varepsilon \nabla_{\xi} \xi\right),
\end{aligned}
$$

where $\varepsilon=g(\xi, \xi), \tilde{\varphi}^{\prime}=\varphi^{\prime f}: f^{-1} T\left(M^{\prime}\right) \rightarrow f^{-1} T\left(M^{\prime}\right)$ is the pullback of $\varphi^{\prime}$ by $f$, and $\nabla^{*}$ is the operator formal adjoint of $\nabla$.

Proof. (sketch) Let $\left\{\xi, E_{\alpha}, \varphi E_{\alpha}: 1 \leq \alpha \leq n\right\}$ be a $\varphi$-basis and let us set $\varepsilon_{\alpha}=g\left(E_{\alpha}, E_{\alpha}\right) \in\{ \pm 1\}$. Then one has

$$
\tau_{\mathcal{H}}(f)=\sum_{\alpha=1}^{n} \varepsilon_{\alpha}\left\{\widetilde{\nabla}_{E_{\alpha}}^{\prime} f_{*} E_{\alpha}-f_{*} \nabla_{E_{\alpha}} E_{\alpha}+\widetilde{\nabla}_{\varphi E_{\alpha}}^{\prime} f_{*} \varphi E_{\alpha}-f_{*} \nabla_{\varphi E_{\alpha}} \varphi E_{\alpha}\right\}
$$

We consider the operator $\nabla^{*}$, the formal adjoint of $\nabla$ (see for example [16], pp. 108-110), thus if $S$ is a tensor of type $(1,1), \nabla^{*} S=-$ trace $\nabla S$. Then, after some computations, we get

$$
\sum_{\alpha=1}^{n} \varepsilon_{\alpha}\left(\nabla_{E_{\alpha}} E_{\alpha}+\nabla_{\varphi E_{\alpha}} \varphi E_{\alpha}\right)=\sum_{\alpha=1}^{n} \varepsilon_{\alpha} \varphi\left[\varphi E_{\alpha}, E_{\alpha}\right]-\varphi \nabla^{*} \varphi-(\operatorname{div} \xi) \xi-\varepsilon \nabla_{\xi} \xi .
$$

Moreover, as $f$ is a CR map,

$$
\begin{aligned}
& \sum_{\alpha=1}^{n} \varepsilon_{\alpha}\left\{\left(\nabla_{f_{*} E_{\alpha}}^{\prime} f_{*} E_{\alpha}+\nabla_{f_{*} \varphi E_{\alpha}}^{\prime} f_{*} \varphi E_{\alpha}\right)=\right. \\
& =\tilde{\varphi}^{\prime}\left(\operatorname{trace}_{\mid \mathcal{H}} f^{*} \nabla^{\prime} \varphi^{\prime}\right)-\left(\operatorname{trace}_{\mid \mathcal{H}} f^{*} \nabla^{\prime} \eta^{\prime}\right) \xi^{\prime}+\sum_{\alpha=1}^{n} \varepsilon_{\alpha}\left\{\tilde{\varphi}^{\prime}\left[f_{*} \varphi E_{\alpha}, f_{*} E_{\alpha}\right]\right\} .
\end{aligned}
$$

Then, Equations (74)-(76) imply

$$
\begin{aligned}
\tau_{\mathcal{H}}(f)= & \sum_{\alpha=1}^{n} \varepsilon_{\alpha}\left\{\left(\tilde{\varphi}^{\prime} f_{*}-f_{*} \varphi\right)\left[\varphi E_{\alpha}, E_{\alpha}\right]\right\}+f_{*}\left(\varphi \nabla^{*} \varphi+(\operatorname{div} \xi) \xi+\varepsilon \nabla_{\xi} \xi\right) \\
& +\tilde{\varphi}^{\prime}\left(\operatorname{trace}_{\mid \mathcal{H}} f^{*} \nabla^{\prime} \varphi^{\prime}\right)-\left(\operatorname{trace}_{\mid \mathcal{H}} f^{*} \nabla^{\prime} \eta^{\prime}\right) \xi^{\prime}
\end{aligned}
$$

By using Equation (77) one gets Equation (73).

Next, if $M$ and $M^{\prime}$ satisfy the $\varphi$-condition, from Equations (75) and (76) we have

$$
\varphi \nabla^{*} \varphi+(\operatorname{div} \xi) \xi+\varepsilon \nabla_{\xi} \xi=0 \quad \text { and } \quad \tilde{\varphi}^{\prime}\left(\operatorname{trace}_{\mid \mathcal{H}} f^{*} \nabla^{\prime} \varphi^{\prime}\right)-\left(\operatorname{trace}_{\mid \mathcal{H}} f^{*} \nabla^{\prime} \eta^{\prime}\right) \xi^{\prime}=0 .
$$

Therefore, we obtain

Corollary 10. ([47]) Let $(M, \varphi, \xi, \eta, g)$ and $\left(M^{\prime}, \varphi^{\prime}, \xi^{\prime}, \eta^{\prime}, g^{\prime}\right)$ be two almost contact semi-Riemannian manifolds, $\operatorname{dim} M=2 n+1$, satisfying the $\varphi$-condition. Then, for any $C R$ map $f: M \rightarrow M^{\prime}$

$$
\tau_{\mathcal{H}}(f)=-\operatorname{trace}(\varphi \nabla \xi) \tilde{\varphi}^{\prime} f_{*} \xi .
$$

If additionally $(M, \varphi, \xi, \eta, g)$ is a contact semi-Riemannian manifold, then

$$
\tau_{\mathcal{H}}(f)=-2 n \varepsilon \tilde{\varphi}^{\prime} f_{*} \xi,
$$

where $\varepsilon=g(\xi, \xi)$. Hence $f$ is Levi harmonic if and only if $f_{*} \xi$ is collinear to $\xi^{\prime}$. 
There are several examples of Levi harmonic maps between almost contact semi Riemannian manifolds. Here we report the following (cf. also [47,94]).

1. Invariant submanifolds and Levi harmonicity

Let $M$ be a submanifold of a $(2 \bar{n}+1)$-dimensional almost contact Riemannian manifold $(\bar{M}, \bar{\varphi}, \bar{\xi}, \bar{\eta}, \bar{g}) . M$ is an invariant submanifold of $\bar{M}$ if $\bar{\varphi}_{x} T_{x}(M) \subset T_{x}(M)$ for any $x \in M$. Two extreme cases may be distinguished (cf. [104]) as:

(I) $\bar{\xi}$ is tangent to $M$ (and then $M$ is odd-dimensional i.e., $\operatorname{dim} M=2 n+1$ ), or

(II) $\bar{\xi}$ is transverse to $M$ (and then $M$ is even-dimensional).

When $\bar{M}$ is a contact Riemannian manifold case II doesn't occur (cf. [2], p. 122). Here we only consider case (I). Then $M$ carries the induced almost contact Riemannian structure $(\eta, \xi, \varphi, g)$ defined by

$$
\eta=i^{*} \bar{\eta}, \quad \bar{\varphi} \circ i_{*}=i_{*} \circ \varphi, \quad g=i^{*} \bar{g},
$$

where $i: M \rightarrow \bar{M}$ is the inclusion. In particular $i$ is a CR map.

If $(\bar{M}, \bar{\eta}, \bar{\zeta}, \bar{\varphi}, \bar{g})$ is an almost contact Riemannian manifold satisfying the $\varphi$-condition, then the submanifold $(M, \eta, \xi, \varphi, g)$ is an almost contact Riemannian manifold satisfying the $\varphi$-condition. Moreover the map $i: M \rightarrow \bar{M}$ is Levi harmonic. The mean curvature $H$ of $i$ is defined by $(2 n+1) H=\alpha(\xi, \xi)$, where $\alpha$ is the second fundamental form of $i$. If additionally $\bar{\xi}$ is geodesic then $i$ is minimal.

To give an explicit example, let $M^{2 m+3}(c)$ be a complete simply connected Sasakian manifold of constant $\varphi$-sectional curvature $c$. As well known $M^{2 m+3}(c)$ is (up to an isometry) one of the Sasakian manifolds $S^{2 m+3}, \mathbb{R}^{2 m+3}$ or $D^{m+1} \times \mathbb{R}$ equipped with Sasakian structures of $\varphi$-sectional curvature $c>-3, c=-3$ and $c<-3$ respectively, where $D^{m+1} \subset \mathbb{C}^{m+1}$ is a simply connected bounded domain. Then, $M^{2 m+1}(c)$ is an invariant submanifold of $M^{2 m+3}(c)$ (cf. [105], p. 328), hence the inclusion $i: M^{2 m+1}(c) \rightarrow M^{2 m+3}(c)$ is Levi harmonic.

\section{Levi harmonicity of Reeb vector fields}

Let $(M, g)$ be a Riemannian manifold. Let $(\bar{\eta}, \bar{\xi}, \bar{\varphi}, \bar{g})$ be the standard contact Riemannian structure on the tangent sphere bundle $T_{1}(M, g)$ which we denoted before by $(\eta, \xi, \varphi, \bar{g})$. Then, it is defined by Equations (63)-(65). In particular the Reeb vector field $\bar{\xi}_{z}=2 u_{z}^{h}, z=(x, u) \in T_{1}(M, g)$. Let $t>0$, consider the $D$-homothetic deformation

$$
\left(\bar{\eta}_{t}=t \bar{\eta}, \bar{\xi}_{t}=(1 / t) \bar{\xi}, \bar{\varphi}_{t}=\bar{\varphi}, \bar{g}_{t}=t \bar{g}+\left(t^{2}-t\right) \bar{\eta} \otimes \bar{\eta}\right) \quad \text { of } \quad(\bar{\eta}, \bar{\xi}, \bar{\varphi}, \bar{g}) .
$$

Then, Equation (78) is a $g$-natural contact Riemannian structure in the sense of [106], and $\bar{g}_{t}$ is the $g$-natural metric on $T_{1} M$ defined by the parameters $a=t / 4, b=c=0$, and $d=\left(t^{2}-t\right) / 4$. In particular, $\bar{g}_{t}$ is of Kaluza-Klein type ([106], p. 1196).

Suppose that $(M, \eta, \xi, \varphi, g)$ is an almost contact Riemannian manifold. Then, by Theorem 6.1 in [106] we get that $\xi:(M, \eta, g) \rightarrow\left(T_{1}(M, g), \bar{\eta}_{t}, \bar{g}_{t}\right)$ is a CR map if and only if $\nabla \xi=-\varphi$ on ker $\eta$. Moreover, since $\bar{g}_{t}$ is of Kaluza-Klein type, by Theorem 6.2 in [106], $(\eta, \xi, \varphi, g)$ is a K-contact Riemannian structure if and only if

$$
\xi \text { is geodesic and } \xi:(M, \eta, g) \rightarrow\left(T_{1}(M, g), \bar{\eta}_{t}, \bar{g}_{t}\right) \text { is a CR map. }
$$

Besides, the differential of $\xi: M \rightarrow T_{1} M$, at $x$, is given by $\xi_{* x} X=X_{z}^{H}+\left(\nabla_{X} \xi\right)_{z}^{T}, \quad z=\left(x, \xi_{x}\right)$. Thus

$$
\xi_{* x}\left(\xi_{x}\right)=\xi_{z}^{H}+\left(\nabla_{\xi} \xi\right)_{z}^{T}=(1 / 2) \bar{\xi}_{z}=(t / 2)\left(\bar{\xi}_{t}\right)_{z}, \quad z=\left(x, \xi_{x}\right),
$$

and

$$
\xi^{*} \bar{\eta}_{t}=\mu \eta, \quad \mu=\left(\xi^{*} \bar{\eta}_{t}\right)(\xi)=t / 2
$$

Summing up, by using also Corollary 10 , we get 
Theorem 32. Let $(M, \eta, \bar{\xi}, \varphi, g)$ be an almost contact Riemannian manifold and let $\left(\bar{\eta}_{t}, \bar{\zeta}_{t}, \bar{\varphi}_{t}, \bar{g}_{t}\right), t>0$, a D-homothetic deformation of the standard contact Riemannian structure of $T_{1}(M, g)$. Then,

$(\eta, g)$ is K-contact structure if and only if $\xi$ is geodesic and $\xi:(M, \eta, g) \rightarrow\left(T_{1}(M, g), \bar{\eta}_{t}, \bar{g}_{t}\right)$ is a CR map.

In particular, if $(\eta, g)$ is K-contact structure, then $\xi:(M, \eta, g) \rightarrow\left(T_{1}(M, g), \bar{\eta}_{t}, \bar{g}_{t}\right)$ is a Levi harmonic map for any $t>0$; moreover, $\xi$ is a pseudohermitian map (isopseudohermitian for $t=2$ ).

Let $\mathbb{S}^{2 n+1} \subset \mathbb{C}^{n+1}$ be the unit sphere endowed with the canonical Sasakian structure $\left(\eta_{0}, \xi_{0}, \varphi_{0}, g_{0}\right)$, hence $\xi_{0}$ is the standard Hopf vector field on $\mathbb{S}^{2 n+1}$. Then

Corollary 11. $\xi_{0}:\left(\mathbb{S}^{2 n+1}, \eta_{0}, g_{0}\right) \rightarrow\left(T_{1} \mathbb{S}^{2 n+1}, \bar{\eta}_{t}, \bar{g}_{0 t}\right)$ is a Levi harmonic map for any $t>0$.

Remark 18. About the harmonicity of Hopf vector fields, Han and Yim [107] proved that these fields, namely, the unit Killing vector fields, are the unique unit vector fields on the unit sphere $\mathbb{S}^{3}$ which define harmonic maps from $\mathbb{S}^{3}$ to $\left(T_{1} \mathbb{S}^{3}, \bar{g}_{0}\right)$, where $\bar{g}_{0}$ is the Sasaki metric. In [108], as a consequence of a more general result, we got in particular that Han-Yim's Theorem is invariant under a three-parameter deformation of the Sasaki metric on $T_{1} \mathbb{S}^{3}$.

Finally, we give a short presentation of the variational treatment of Levi harmonicity. Let $(M, \eta, \xi, \varphi, g)$ be a $(2 n+1)$-dimensional almost contact Riemannian manifold and $\left(M^{\prime}, g^{\prime}\right)$ a Riemannian manifold. If $\Omega \subset M$ is a relatively compact domain we set

$$
E_{\Omega}(f)=\frac{1}{2} \int_{\Omega} \operatorname{trace}_{g}\left(\Pi_{\mathcal{H}} f^{*} g^{\prime}\right) d v_{g}
$$

for any $f \in C^{\infty}\left(M, M^{\prime}\right)$. Then we obtain the following ([47], Theorem 6.1):

Theorem 33. Let $\Omega \subset M$ be a relatively compact domain. A $C^{\infty}$ map $f: M \rightarrow M^{\prime}$ is a critical point for the energy functional $E_{\Omega}: C^{\infty}\left(M, M^{\prime}\right) \rightarrow \mathbb{R}$ defined by (79) if and only if $\tau_{\mathcal{H}}(f)=f_{*}\left\{\nabla_{\xi} \xi+\operatorname{div}(\xi) \xi\right\}$. If $f: M \rightarrow M^{\prime}$ be an immersion and a critical point of $E_{\Omega}$, then $f$ is Levi harmonic if and only if the Reeb field $\xi$ is geodesic and divergence free.

Remark 19. The many ramifications of harmonicity (subelliptic harmonic, contact harmonic, Levi harmonic, and pseudoharmonic maps) seem to indicate that the theory of harmonic maps has reached a stage of mannerism. However, the mentioned ramifications (to which one may add p-harmonic and exponentially harmonic maps, Gromov's tangentially harmonic maps and harmonic maps from Finslerian manifolds (cf. references in [47])) are but a measure of the enormous success enjoyed by the theory.

\section{Some Open Problems}

Question 1. (related to the Section 2.3) It is an open problem, to our knowledge, to find examples of non-Sasakian contact semi-Riemannian manifolds which satisfy Equation (23), or to give a proof that an arbitrary contact semi-Riemannian manifolds satisfying Equation (23) is Sasakian.

Question 2. (related to the Section 2.4) In dimension $\geq 5$, it is an open problem, to our knowledge, the existence of non trivial semi-Riemannian contact Ricci solitons.

Question 3. (related to Section 3.2) Study the geometry of an almost contact (semi) Riemannian structure $(\eta, \xi, \varphi, g)$ when $\eta$ defines a pseudohermitian structure.

Question 4. (related to the Section 3.4) In dimension $\geq 5$, it is an open problem to see if the Olszak's result holds for a general non-degenerate almost CR manifold. 
Question 5. (related to the Section 4 and Definition 4$)$ Let $(\mathcal{H}, J, \theta)$ be a pseudohermitian $C R$ structure on a simply connected three-manifold.

- If $(\mathcal{H}, J, \theta)$ is homogeneous and non-degenerate, then Theorem 21 gives a complete classification.

- If $(\mathcal{H}, J, \theta)$ is a homogeneous Levi-flat pseudohermitian CR structure induced by a homogeneous almost $\alpha$-coKähler structure, then we have a complete classification (cf. [33,41]).

- If $(\mathcal{H}, J, \theta)$ is an arbitrary homogeneous Levi-flat pseudohermitian CR structure, we do not know a classification.

So, a natural open problem is: Classify all simply connected three-manifolds which admit a homogeneous Levi-flat pseudohermitian CR structure.

Question 6. In the Riemannian case, S.H. Chun et al. [90] proved the following: if $(M, g)$ is an Einstein Riemannian manifold, then the standard contact Riemannian manifold on $T_{1}(M, g)$ is $H$-contact if and only if $(M, g)$ is 2-stein. Then, a natural open problem (related to the Section 5.2) is the following.

Let $(M, g)$ be an Einstein semi-Riemannian manifold. When is the standard non-degenerate almost $C R$ structure on $T_{\mathcal{\varepsilon}}(M, g) H$-contact?

Question 7. Another open problem (related to the Section 5.2) is the following. Let $(M, g)$ be a emi-Riemannian manifold (or an Einstein semi-Riemannian manifold). When is the Reeb vector field of (the standard non-degenerate almost $C R$ structure on) $T_{\varepsilon}(M, g)$ an i.h.t.?

Funding: Author supported by funds of Universitá del Salento.

Acknowledgments: The author would like to thank the anonymous referees for their useful comments.

Conflicts of Interest: The author declares no conflict of interest.

\section{References}

1. Geiges, H. A brief history of contact geometry and topology. Expo. Math. 2001, 19, 25-53. [CrossRef]

2. Blair, D.E. Riemannian Geometry of Contact and Symplectic Manifolds, 2nd ed.; Progress in Mathematics 203; Birkhäuser: Boston, MA, USA, 2010.

3. Boyer, C.P.; Galicki, K. Sasakian geometry. In Oxford Mathematical Monographs; Oxford University Press: Oxford, UK, 2008.

4. Takahashi, T. Sasakian manifold with pseudo-Riemannian metrics. Tohoku Math. J. 1969, 21, 271-290. [CrossRef]

5. Duggal, K.L. Space time manifolds and contact structures. Int. J. Math. Math. Sci. 1990, 13, 545-554. [CrossRef]

6. Baum, H. Twistor and Killing spinors in Lorentzian geometry, Global analysis and harmonic analysis (Marseille-Luminy, 1999). Séminaires Congrés 2000, 4, 35-52.

7. Calvaruso, G.; Perrone, D. Contact pseudo metric manifolds. Differ. Geom. Appl. 2010, 28, 615-634. [CrossRef]

8. Calvaruso, G.; Perrone, D. Erratum to: “Contact pseudo-metric manifolds. Differential Geom. Appl. 28 (2010), 615-634". Differ. Geom. Appl. 2013, 31, 836-837. [CrossRef]

9. Chern, S.S.; Moser, J. Real hypersurfaces in complex manifolds. Acta Math. 1974, 133, 219-271. [CrossRef]

10. Tanaka, N. A Differential Geometric Study on Strongly Pseudo-Convex Manifolds; Kinokuniya Book Store Co., Ltd.: Tokyo, Japan, 1975.

11. Webster, S. Pseudohermitian structures on a real hypersurface. J. Differ. Geom. 1978, 13, 25-41. [CrossRef]

12. Dragomir, S.; Tomassini, G. Differential Geometry and Analysis on CR Manifolds; Progress in Mathematics 246; Birkhäuser: Boston, MA, USA; Basel, Switzerland; Berlin, Germany, 2006.

13. Alekseevsky, D.V.; Cortés, V.; Galaev, A.S.; Leistner, T. Cones over pseudo-Riemannian manifolds and their holonomy. J. Reine Angew. Math. 2009, 635, 23-69. [CrossRef]

14. Bohle, C. Killing spinors on Lorentzian manifolds. J. Geom. Phys. 2003, 45, 285-308. [CrossRef]

15. Perrone, D. Contact metric manifolds whose characteristic vector fied is a harmonic vector field. Diff. Geom. Appl. 2004, 20, 367-378. [CrossRef] 
16. Dragomir, S.; Perrone, D. Harmonic Vector Fields: Variational Principles and Differential Geometry; Elsevier, Inc.: Amsterdam, The Netherlands, 2012.

17. Calvaruso, G.; Perrone, D. H-contact semi-Riemannian manifolds. J. Geom. Phys. 2013, 71, 11-21. [CrossRef]

18. Perrone, D. Harmonic characteristic vector fields on contact metric three-manifolds. Bull. Aust. Math. Soc. 2003, 67, 305-315. [CrossRef]

19. Cho, J.T. $\eta$-parallel H-contact 3-manifolds. Bull. Korean Math. Soc. 2018, 55, 1013-1022. [CrossRef]

20. Tanno, S. Variational problems on contact Riemannian manifolds. Trans. Am. Math. Soc. 1989, 314, 349-379. [CrossRef]

21. Boyer, C.P.; Galicki, K. Einstein manifold and contact geometry. Proc. Am. Math. Soc. 2001, 129, $2419-2430$. [CrossRef]

22. Perrone, D. Curvature of K-contact semi-Riemannian manifolds. Can. Math. Bull. 2014, 57, 401-412. [CrossRef]

23. Gomez, R.R. Lorentzian Sasaki-Einstein metrics on connected sums of $S^{2} \times S^{3}$. Geom. Dedicata 2011, 150, 249-255. [CrossRef]

24. Nouhaud, O. Transformations infinitesimales harmoniques. C. R. Acad. Sci. Paris 1972, 274, 573-576.

25. Stepanov, S.E.; Shandra, I.G. Geometry of infinitesimal harmonic transformations. Ann. Glob. Anal. Geom. 2003, 24, 291-299. [CrossRef]

26. Perrone, D. Geodesic Ricci solitons on unit tangent sphere bundles. Ann. Glob. Anal. Geom. 2013, 44, 91-103. [CrossRef]

27. Gil-Medrano, O.; Hurtado, A. Spacelike energy of timelike unit vector fields on a Lorentzian manifold. J. Geom. Phys. 2004, 51, 82-100. [CrossRef]

28. Chow, B.; Knopf, D. The Ricci Flow: An Introduction; Mathematical Surveys and Monographs Volume 110; American Mathematical Society: Providence, RI, USA, 2004.

29. Brozos-Vazquez, M.; Calvaruso, G.; Garcia-Rio, E.; Gavino-Fernandez, S. Three-dimensional Lorentzian homogeneous Ricci solitons. Israel J. Math. 2012, 188, 385-403. [CrossRef]

30. Stepanov, S.E.; Shelepova, V.N. A remark on Ricci solitons. Mat. Zametki 2009, 86, 474-477. (In Russian). Translation in Math. Notes 2009, 86, 447-450.

31. Cho, J.T. Notes on contact Ricci solitons. Proc. Edimb. Math. Soc. 2011, 54, 47-53. [CrossRef]

32. Calvaruso, G.; Perrone, A. Natural almost contact structures and their 3D homogeneous models. Math. Nachr. 2016, 289, 1370-1385. [CrossRef]

33. Perrone, D. Classification of homogeneous almost $\alpha$-coKaehler three-manifolds. Differ. Geom. Appl. 2018, 59, 66-90. [CrossRef]

34. Perrone, D. Contact pseudo-metric manifolds of constant curvature and CR geometry. Results Math. 2014, 66, 213-225. [CrossRef]

35. Barletta, E.; Dragomir, S. Differential equations on contact Riemannian manifolds. Annali della Scuola Normale Superiore di Pisa 2001, 30, 63-95.

36. Blair, D.; Dragomir, S. Pseudohermitian geometry on contact Riemannian manifolds. Rendiconti di Matematica 2002, 22, 275-341.

37. Boeckx, E.; Cho, J.T. Pseudo-Hermitian symmetries. Isr. J. Math. 2008, 166, 125-145. [CrossRef]

38. Barros, E.; Romero, A. Indefinite Kaehler manifolds. Math. Ann. 1982, 261, 55-62. [CrossRef]

39. Bejancu, A.; Duggal, K.L. Real hypersurfaces of indefinite Kaehler manifolds. Int. J. Math. Math. Sci. 1993, 16, 545-556. [CrossRef]

40. Goldberg, S.I.; Yano, K. Integrability of almost cosymplectic structures. Pac. J. Math. 1969, 31, $373-381$. [CrossRef]

41. Perrone, D. Left invariant almost $\alpha$-coKähler structure structures on 3D semidirect product Lie groups. Int. J. Geom. Methods Mod. Phys. 2018. [CrossRef]

42. Boutet de Monvel, L. Integration des equations de Cauchy-Riemann induites formelles. Séminaire Équations aux Dérivées Partielles 1974-1975, 9, 1-13.

43. Burns, D.M.; Epstein, C. Embeddability for three-dimensional CR-manifolds. J. Am. Math. Soc. 1990, 3, 809-841. [CrossRef]

44. Lempert, L. Embeddings of three-dimensional Cauchy-Riemann manifolds. Math. Ann. 1994, 300, 1-15. [CrossRef] 
45. Chanillo, S.; Chiu, H.; Yang, P. Embeddability for the three dimensional CR manifolds and CR Yamabe invariants. Duke Math. J. 2012, 161, 2909-2921. [CrossRef]

46. Chanillo, S.; Chiu, H.; Yang, P. Embedded three-dimensional CR manifolds and the non-negativity of Paneitz operators. Geom. Anal. Math. Relativ. Nonlinear Part. Differ. Eq. 2012, 599, 65-82.

47. Dragomir, S.; Perrone, D. Levi harmonic maps of contact Riemannian manifols. J. Geom. Anal. 2014, 24, 1233-1275. [CrossRef]

48. Perrone, D. On the standard nondegenerate almost CR structures of tangent hyperquadrics bundles. Geom. Dedicata 2016, 185, 15-33. [CrossRef]

49. Tanno, S. The Bochner type curvature tensor of contact Riemannian structure. Hokkaido Math. J. 1990, 19, 55-66. [CrossRef]

50. Lee, J.M. Pseudo-Einstein structures on CR manifolds. Am. J. Math. 1988, 110, 157-178. [CrossRef]

51. Chern, S.S.; Hamilton, R.S. On Riemannian Metrics Adapted to Three-DimenDional Contact Manifolds; Lecture Notes in Mathematics 1111; Springer: Berlin/Heidelberg, Germany; New York, NY, USA, 1985; pp. $279-305$.

52. Goldberg, S.I.; Perrone, D.; Toth, G. Curvature of Contact Three-Manifolds with Critical Metrics; Lecture Notes in Mathematics 1410; Springer: Berlin/Heidelberg, Germany; New York, NY, USA, 1989; pp. 212-222.

53. Duggal, K.L.; Bejancu, A. Lightlike Submanifolds of Semi-Riemannian Manifolds and Applications; Kluwer Academic Publishers: Dordrecht, The Netherlands, 1996; Volume 364.

54. Perrone, D. Torsion and critical metrics on contact three-manifolds. Kodai Math. J. 1990, 13, 88-100. [CrossRef]

55. Nagano, T. A problem on the existence of an Einstein metric. J. Math. Soc. Japan 1967, 19, 30-31. [CrossRef]

56. Boothby, W.M.; Wang, H.C. On contact manifolds. Ann. Math. 1958, 68, 721-734. [CrossRef]

57. Goldberg, S.I. Rigidity of positively curved contact manifolds. J. Lond. Math. Soc. 1967, 42, $257-263$. [CrossRef]

58. Tanno, S. Harmonic forms and Betti numbers of certain contact Riemannian manifolds. J. Math. Soc. Jpn. 1967, 19, 308-316. [CrossRef]

59. Blair, D.E. On the non-existence of flat contact metric structures. Tohoku Math. J. 1976, 28, 373-379. [CrossRef]

60. Olszak, Z. On contact metric manifolds. Tohoku Math. J. 1979, 31, 247-253. [CrossRef]

61. Blair, D.E.; Koufogiorgos, T.; Papantoniou, B.J. Contact metric manifolds satisfying a nullity condition. Israel J. Math. 1995, 91, 189-214. [CrossRef]

62. Boeckx, E. A full classification of contact metric $(\kappa, \mu)$-spaces. Rend. Mat. Appl. 2000, 44, 212-219.

63. Barletta, E.; Dragomir, S. Jacobi fields of the Tanaka-Webster connection on Sasakian manifolds. Kodai Math. J. 2006, 29, 406-454. [CrossRef]

64. Sakamoto, K.; Takemura, Y. On almost contact structures belonging to a CR-structure. Kodai Math. J. 1980, 3, 144-161. [CrossRef]

65. Sakamoto, K.; Takemura, Y. Curvature invariants of CR-manifolds. Kodai Math. J. 1981, 4, 251-265. [CrossRef]

66. Alekseevsky, D.V.; Spiro, A.F. Invariant CR structures on compact homogeneous manifolds. Hokkaido Math. J. 2003, 32, 209-276. [CrossRef]

67. Perrone, D. Homogeneous contact Riemannian three-manifolds. Illinois J. Math. 1998, 42, 243-256.

68. Geiges, H. Normal contact structures on 3-manifolds. Tohoku Math. J. 1997, 49, 415-422. [CrossRef]

69. Goldberg, S.I.; Perrone, D. Contact 3-manifolds with positive scalar curvature. Contemp. Math. 1992, 127, 59-68.

70. Hamilton, R.S. Three-manifolds with positive Ricci curvature. J. Differ. Geom. 1992, 17, 255-306. [CrossRef]

71. Perrone, D. Taut contact circles and bi-contact metric structures on three-manifolds. Ann. Global Anal. Geom. 2017, 52, 213-235. [CrossRef]

72. Geiges, H.; Gonzalo, J. Contact geometry and complex surfaces. Invent. Math. 1995, 121, 147-209. [CrossRef]

73. Geiges, H.; Gonzalo, J. Contact circles on 3-manifolds. J. Differ. Geom. 1997, 46, 236-286. [CrossRef]

74. Lotta, A. Non existence of homogeneous contact metric manifolds of nonpositive curvature. Tohoku Math. J. 2010, 62, 575-578. [CrossRef]

75. Boeckx, E.; Cho, J.T. Locally symmetric contact metric manifolds. Monatsh. Math. 2006, 148, $269-281$. [CrossRef]

76. Correa, E.M. Homogeneous contact manifolds and resolutions of Calabi Yau cones. arXiv 2018, arXiv:1801.02763v2.

77. Perrone, D.; Vanhecke, L. Five-dimensional homogeneous contact manifolds and related problems. Tohoku Math. J. 1991, 43, 243-248. [CrossRef] 
78. Alekseevsky, D.V.; Spiro, A.F. Compact Homogeneous CR Manifolds. J. Geom. Anal. 2002, 12, $183-201$. [CrossRef]

79. Burns, D.; Shnider, S. Spherical hypersurfaces in complex manifolds. Invent. Math. 1976, 33, $223-246$. [CrossRef]

80. Lehmann, R.; Feldmueller, D. Homogeneous CR hypersurface structures on spheres. Annali della Scuola Normale Superiore di Pisa 1987, 14, 513-525.

81. Huckleberry, A.; Richthofer, W. Recent Developments in Homogeneous CR Hypersurfaces. In Contributions to Several Complex Variables; Aspects of Mathematics E9; Vieweg+Teubner Verlag: Braunschweig, Germany, 1986.

82. Dileo, G.; Lotta, A. A classification of spherical symmetric CR manifolds. Bull. Aust. Math. Soc. 2009, 80, 251-274. [CrossRef]

83. Tanno, S. The standard CR structure on the unit tangent bundle. Tohoku Math. J. 1992, 44, 535-543. [CrossRef]

84. Dragomir, S.; Perrone, D. On the geometry of tangent hyperquadric bundles: CR and pseudo harmonic vector fields. Ann. Glob. Anal. Geom. 2006, 30, 211-238. [CrossRef]

85. Satoh, T.; Sekizawa, M. Curvatures of tangent hyperquadric bundles. Differ. Geom. Appl. 2011, 29, $255-260$. [CrossRef]

86. Dajczer, M.; Nomizu, K. On sectional curvature of indefinite metrics. Math. Ann. 1980, 247, $279-282$. [CrossRef]

87. Nomizu, K. Remarks on sectional curvature of an indefinite metric. Proc. Am. Math. Soc. 1983, 89, $473-476$. [CrossRef]

88. Calvaruso, G.; Perrone, D. H-contact unit-tangent sphere bundles. Rocky Mt. J. Math. 2007, 37, $1435-1457$. [CrossRef]

89. Calvaruso, G.; Perrone, D. Homogeneous and H-contact unit tangent sphere bundles. J. Aust. Math. Soc. 2010, 88, 323-337. [CrossRef]

90. Chun, S.H.; Park, J.H.; Sekigawa, K. H-contact unit tangent sphere bundles of Einstein manifolds. Q. J. Math. 2011, 62, 59-69. [CrossRef]

91. Chun, S.H.; Park, J.H.; Sekigawa, K. H-contact unit tangent sphere bundles of four-dimensional Riemannian manifolds. J. Aust. Math. Soc. 2011, 91, 243-256. [CrossRef]

92. Chun, S.H.; Pak, H.K.; Park, J.H.; Sekigawa, K. A remark on H-contact unit tangent sphere bundles. J. Korean Math. Soc. 2011, 48, 329-340. [CrossRef]

93. Loiudice, E.; Lotta, A. On the classification of contact metric $(\kappa, \mu)$-spaces via tangent hyperquadric bundles. Math. Nachr. 2018, 291, 1851-1858. [CrossRef]

94. Perrone, D. Remarks on Levi harmonicity of contact semi-Riemannian manifolds. J. Korean Math. Soc. 2014, 51, 881-895. [CrossRef]

95. Strichartz, R.S. Sub-Riemannian geometry. J. Differ. Geom. 1986, 24, 221-263. [CrossRef]

96. Barletta, E.; Dragomir, S.; Urakawa, H. Pseudoharmonic maps from non degenerate CR manifolds to Riemannian manifolds. Indiana Univ. Math. J. 2001, 50, 719-746. [CrossRef]

97. Petit, R. Harmonic maps and strictly pseudoconvex CR manifolds. Commun. Anal. Geom. 2002, 41, 575-610. [CrossRef]

98. Dragomir, S.; Petit, R. Contact harmonic maps. Differ. Geom. Appl. 2012, 30, 65-84. [CrossRef]

99. Konderak, J.J.; Wolak, R.A. Transversally harmonic maps between manifolds with Riemannian manifolds. Q. J. Math. 2003, 54, 335-354. [CrossRef]

100. Fuglede, B. Harmonic morphisms between semi-Riemannian manifolds. Ann. Acad. Sci. Fenn. 1996, 21, 31-50.

101. Baird, P.; Wood, J.C. Harmonic Morphisms between Riemannian Manifolds; London Math. Society Monographs; Oxford Science Publications, Clarendon Press: Oxford, UK, 2003.

102. Rawnsley, J.H. f-structures, f-twistor spaces and harmonic maps. In Geometry Seminar L. Bianchi; Lecture Notes in Mathematics; Springer: Berlin/Heidelberg, Germany, 1985; pp. 84-159.

103. Kim, J.H.; Park, J.H.; Sekigawa, K. A generalization of contact metric manifolds. Balkan J. Geom. Appl. 2014, 19, 94-105.

104. Yano, K.; Ishihara, S. Invariant submanifolds of an almost contact manifold. Kodai Math. Sem. Rep. 1969, 21, 350-364. [CrossRef]

105. Yano, K.; Kon, M. Structures on Manifolds; Series in Pure Mathematics; World Scientific Publishing Co.: Singapore, 1984; Volume 3. 
106. Perrone, D. Minimality, harmonicity and CR geometry for Reeb vector fields. Int. J. Math. 2010, 21, 1189-1218. [CrossRef]

107. Han, S.D.; Yim, J.W. Unit vector fields on spheres which are harmonic maps. Math. Z. 1998, 227, 83-92 [CrossRef]

108. Perrone, D. Unit vector fields on real space forms which are harmonic maps. Pac. J. Math. 2009, 239, 89-104. [CrossRef]

(C) 2019 by the author. Licensee MDPI, Basel, Switzerland. This article is an open access article distributed under the terms and conditions of the Creative Commons Attribution (CC BY) license (http://creativecommons.org/licenses/by/4.0/). 